MATHEMATICS OF COMPUTATION

Volume 80, Number 274, April 2011, Pages 781-819

S $0025-5718(2010) 02429-9$

Article electronically published on December 8, 2010

\title{
MIXED FORMULATION, APPROXIMATION AND DECOUPLING ALGORITHM FOR A PENALIZED NEMATIC LIQUID CRYSTALS MODEL
}

\author{
V. GIRAULT AND F. GUILLÉN-GONZÁLEZ
}

\begin{abstract}
A linear fully discrete mixed scheme, using $C^{0}$ finite elements in space and a semi-implicit Euler scheme in time, is considered for solving a penalized nematic liquid crystal model (of the Ginzburg-Landau type). We prove: 1) unconditional stability and convergence towards weak solutions, and 2) first-order optimal error estimates for regular solutions (but without imposing the well-known global compatibility condition for the initial pressure in the Navier-Stokes framework). These results are valid in a general connected polygon or in a Lipschitz polyhedral domain (without any constraints on its angles).

Finally, since the scheme couples the unknowns, we propose several algorithms for decoupling the computation of these unknowns and establish their rates of convergence in convex domains when the mesh size is sufficiently small compared to the time step.
\end{abstract}

\section{INTRODUCTION}

In this work, we discretize a system of partial differential equations related to the motion of a nematic liquid crystal. The orientation vector of the molecules is normalized by means of a penalty argument, thus leading to a simplified Ericksen-Leslie model with the Ginzburg-Landau approximation (see Béthuel, Brezis \& Hélein [5] and Chen [9]).

Let us consider a simplified version of the Ericksen-Leslie model, introduced by Lin in 223] and analyzed by Lin and Liu in [24, 25] who used a modified Galerkin approach, and by Shkoller 35] who relied on a contraction mapping argument coupled with appropriate energy estimates. This model is a modified Navier-Stokes system that takes into account the liquid crystal nature of the fluid, coupled with the Ginzburg-Landau equations. A more complete version of this Ericksen-Leslie model has been studied by Coutand and Shkoller in [11, where local well-posedness (or global well-posedness for small data) is proven.

The fluid is confined in an open bounded connected domain $\Omega \subset \mathbb{R}^{N}(N=2$ or 3) with boundary $\partial \Omega$. The unknowns are the time-dependent divergence-free velocity field $\boldsymbol{u}(t, \boldsymbol{x})$, the pressure $p(t, \boldsymbol{x})$ of the fluid and the director field $\boldsymbol{d}(t, \boldsymbol{x})$

Received by the editor February 11, 2009 and, in revised form, March 1, 2010.

2010 Mathematics Subject Classification. Primary 35Q35, 65M12, 65M15, 65M60.

Key words and phrases. Nematic liquid crystal, Ginzburg-Landau penalization, mixed formulation, finite element method, convergence, stability, error estimates, decoupling algorithm.

The second author was partially supported by DGI-MEC (Spain), Grant MTM2006-07932 and by Junta de Andalucía project P06-FQM-02373.

(C)2010 American Mathematical Society Reverts to public domain 28 years from publication 
representing the orientation of the liquid crystal molecules; thus $\boldsymbol{d}$ is a unit vector:

$$
|\boldsymbol{d}|=1 \text {. }
$$

Both theoretically and numerically, enforcing the constraint $|\boldsymbol{d}|=1$ is difficult. Here we choose to satisfy it approximately by imposing the weaker condition $|\boldsymbol{d}| \leq 1$ and introducing the following penalty function (of Ginzburg-Landau type) in the equation of conservation of angular momentum

$$
\boldsymbol{f}(\boldsymbol{d})=\frac{1}{\varepsilon^{2}}\left(|\boldsymbol{d}|^{2}-1\right) \boldsymbol{d}, \varepsilon>0
$$

where $\varepsilon$ is the penalty parameter. This penalty function was proposed by many authors, in particular, because it has a potential structure; i.e., there exists a potential function

$$
F(\boldsymbol{d})=\frac{1}{4 \varepsilon^{2}}\left(|\boldsymbol{d}|^{2}-1\right)^{2},
$$

such that

$$
\forall \boldsymbol{d} \in \mathbb{R}^{N}, \boldsymbol{f}(\boldsymbol{d})=\nabla_{\boldsymbol{d}}(F(\boldsymbol{d})) .
$$

Interestingly, it turns out that, in the penalized model, the weaker constraint $|\boldsymbol{d}| \leq$ 1 is a consequence of a maximum principle for the Ginzburg-Landau equation. Indeed, we shall see that if this constraint is satisfied at initial time and on $\partial \Omega$ for all time, then it is also satisfied in the interior of $\Omega$ at any time.

Accordingly, we consider the following penalized model in $] 0, T[\times \Omega$ :

$$
\begin{aligned}
|\boldsymbol{d}| \leq 1, \quad \partial_{t} \boldsymbol{d}+\boldsymbol{u} \cdot \nabla \boldsymbol{d}+\gamma(\boldsymbol{f}(\boldsymbol{d})-\Delta \boldsymbol{d}) & =\mathbf{0}, \\
\partial_{t} \boldsymbol{u}+\boldsymbol{u} \cdot \nabla \boldsymbol{u}-\nu \Delta \boldsymbol{u}+\nabla p+\lambda \nabla \cdot(\nabla \boldsymbol{d} \odot \nabla \boldsymbol{d}) & =\mathbf{0}, \\
\nabla \cdot \boldsymbol{u} & =0, \\
\boldsymbol{u}_{\mid \partial \Omega}=\mathbf{0}, \quad \boldsymbol{d}_{\mid \partial \Omega} & =l, \\
\boldsymbol{u}_{\mid t=0}=\boldsymbol{u}_{0}, \quad \boldsymbol{d}_{\mid t=0} & =\boldsymbol{d}_{0} .
\end{aligned}
$$

The functions $\boldsymbol{u}_{0}$ and $\boldsymbol{d}_{0}: \Omega \rightarrow \mathbb{R}^{N}$ are, respectively, the initial velocity and director fields, and $\boldsymbol{l}:] 0, T\left[\times \partial \Omega \rightarrow \mathbb{R}^{N}\right.$ is the Dirichlet boundary data for the director field $\boldsymbol{d}$. Concerning the coefficients, $\nu>0$ represents the viscosity of the fluid, $\lambda>0$ is an elasticity constant and $\gamma>0$ is a relaxation-time constant. Here we use the tensor notation

$$
\nabla \boldsymbol{d} \odot \nabla \boldsymbol{d}=(\nabla \boldsymbol{d})^{t} \nabla \boldsymbol{d}
$$

where $(\nabla \boldsymbol{d})^{t}$ denotes the transpose of $\nabla \boldsymbol{d}=\left(\partial d_{i} / \partial x_{j}\right)_{i, j}$.

In [17, Guillén-González and Rojas-Medar study the asymptotic limit of (1.4)(1.8) as $\varepsilon$ goes to zero, arriving at a model with the restriction (1.1), $|\nabla \boldsymbol{d}|^{2} \boldsymbol{d}$ being its associated Lagrange multiplier. Indeed, when $\varepsilon \rightarrow 0$ one finds a limit problem, with (1.4) replaced by

$$
|\boldsymbol{d}|=1, \quad \partial_{t} \boldsymbol{d}+\boldsymbol{u} \cdot \nabla \boldsymbol{d}-\gamma\left(\Delta \boldsymbol{d}+|\nabla \boldsymbol{d}|^{2} \boldsymbol{d}\right)=\mathbf{0} .
$$

However, as mentioned above, enforcing (1.1) is difficult and therefore, in this article, we only study the penalized model (1.4)-(1.8) with a fixed penalty parameter $\varepsilon$. In consequence, the generic constants involved in the numerical analysis may depend (exponentially) on $\varepsilon$. 
As far as numerical approximation is concerned, Liu and Walkington propose and study two numerical schemes for solving (1.4)-(1.8) in the case where the Dirichlet boundary data $\boldsymbol{l}$ for the director field $\boldsymbol{d}$ does not depend on time; for both schemes, they derive error estimates when the solutions are sufficiently smooth. In [29], they introduce a scheme that discretizes $\boldsymbol{d}$ in a finite element subspace of $H^{2}(\Omega)^{N}$; this means that the discrete functions are globally $\mathcal{C}^{1}$. From a practical point of view, implementing a scheme that uses $\mathcal{C}^{1}$ finite elements is not easy, the more so in three dimensions. To avoid this regularity, they relax it in 30. by switching to a mixed formulation where the gradient tensor $\nabla \boldsymbol{d}$ is introduced as an auxiliary unknown tensor. This allows them to work with finite element subspaces of $H^{1}(\Omega)$. However, in this approach, the components of the discrete mixed finite element functions are strongly coupled, and as the problem is nonlinear, this coupling is not desirable.

In this article, we propose a different mixed formulation of (1.4) (1.8) where $\Delta \boldsymbol{d}$ is introduced as an auxiliary variable. The advantage of using the Laplace instead of the gradient operator is that the Laplace operator involves less unknowns: $M$ versus $\frac{1}{2} M(M-1)$. Furthermore, the Laplace operator can be discretized in standard finite element subspaces of $L^{2}(\Omega)^{N}$, whereas the gradient operator is discretized by [30] in Raviart-Thomas mixed finite element subspaces of $H(\operatorname{div}, \Omega)$. We construct a fully discrete time-stepping linearized Euler scheme that is unconditionally stable and convergent towards weak solutions of the penalized problem (1.4)-(1.8) and we derive optimal error estimates when the solution is sufficiently smooth. Since the scheme is implicit and couples the unknowns, we propose several algorithms for decoupling the computation of these unknowns, and we establish their rates of convergence when the mesh size is sufficiently small compared to the time step.

We refer to Prohl 32 for the numerical analysis of a splitting in time projection scheme in the limit model (1.5)-(1.9).

In [26], two linearized numerical algorithms are presented. The first of them uses an implicit backward Euler approximation to discretize the time derivative and the second one uses a characteristic method, but both schemes consider $C^{0}$ finite elements in space. Numerical experiments show that both schemes recover the numerical results obtained in [29, but no analytical justification for the stability of these schemes is proposed.

In a recent publication, Becker, Feng and Prohl [4 present two nonlinear fully discrete finite element schemes. The first scheme, applied to the penalized model (1.4)-(1.8), uses $-\boldsymbol{\Delta} \boldsymbol{d}+\boldsymbol{f}_{\varepsilon}(\boldsymbol{d})$ as an auxiliary variable and is unconditionally stable (uniformly with respect to $\varepsilon$ ) and convergent. The second algorithm discretizes directly the limit problem (1.5)-(1.9); it is conditionally stable, but the convergence remains an open problem. Both schemes use $C^{0}$ finite elements for all unknowns.

Finally, a linearized fully discrete $C^{0}$ finite elements scheme that is conditionally stable (uniformly with respect to $\varepsilon$ ) and convergent has been recently analyzed by Guillén-González and Gutiérrez-Santacreu in [19].

1.1. Summary of results. For simplicity, we denote the spaces of vector-valued functions $L^{2}(\Omega)^{N}, H^{1}(\Omega)^{N}$, etc. by boldface letters $\boldsymbol{L}^{2}, \boldsymbol{H}^{1}$, etc.

By considering $\boldsymbol{w}=-\mu \Delta \boldsymbol{d}$ (with $\mu=\sqrt{\lambda}$ ) as an auxiliary variable, using this relation as a constraint and suitably modifying the pressure, we arrive at the following variational formulation equivalent to problem (1.4)-(1.8): Find $(\boldsymbol{u}, p, \boldsymbol{w}, \boldsymbol{d})$ satisfying for a.e. $t \in(0, T)$, 


$$
\begin{array}{ll}
d_{t}(\boldsymbol{u}, \boldsymbol{v})+\nu(\nabla \boldsymbol{u}, \nabla \boldsymbol{v})+(\boldsymbol{u} \cdot \nabla \boldsymbol{u}, \boldsymbol{v})-\mu\left((\nabla \boldsymbol{d})^{t} \boldsymbol{w}, \boldsymbol{v}\right)- & (p, \nabla \cdot \boldsymbol{v})=0, \\
& \forall \boldsymbol{v} \in \boldsymbol{H}_{0}^{1}(\Omega), \\
\gamma(\boldsymbol{w}, \boldsymbol{e})+\mu\left\{d_{t}(\boldsymbol{d}, \boldsymbol{e})+(\boldsymbol{u} \cdot \nabla \boldsymbol{d}, \boldsymbol{e})+\gamma(\boldsymbol{f}(\boldsymbol{d}), \boldsymbol{e})\right\}=0, & \forall \boldsymbol{e} \in \boldsymbol{L}^{2}(\Omega), \\
(\nabla \cdot \boldsymbol{u}, q)=0, \quad \forall q \in L_{0}^{2}(\Omega), & \\
\mu(\nabla \boldsymbol{d}, \nabla \boldsymbol{g})-(\boldsymbol{w}, \boldsymbol{g})=0, \quad \forall \boldsymbol{g} \in \boldsymbol{H}_{0}^{1}(\Omega), &
\end{array}
$$

together with the Dirichlet boundary conditions and initial conditions (1.7) and (1.8) on $\boldsymbol{u}$ and $\boldsymbol{d}$. Assuming that $\Omega$ is a polygon or a Lipschitz polyhedron, we discretize this formulation in time with a uniform partition of $[0, T]$ and time step $k$, and in space with a stable pair of finite elements for $(\boldsymbol{u}, p)$ such as the mini-element $\left(\mathbb{P}_{1}+\mathbb{B}\right) \times \mathbb{P}_{1}$ on a triangulation with mesh-size $h$, a continuous piecewise $\mathbb{P}_{1}$ space for $\boldsymbol{d}$ and a piecewise $\mathbb{P}_{0}$ space for $\boldsymbol{w}$. Let $\left(U_{0 h}, P_{h}\right) \subset \boldsymbol{H}_{0}^{1}(\Omega) \times L_{0}^{2}(\Omega), D_{h} \subset \boldsymbol{H}^{1}(\Omega)$ and $W_{h} \subset \boldsymbol{L}^{2}(\Omega)$ denote the discrete spaces for $(\boldsymbol{u}, p), \boldsymbol{d}$ and $\boldsymbol{w}$ respectively, and let $t_{n}=n k$ denote the discrete points in time. We propose the following fully discrete scheme, starting from a suitable approximation $\left(\boldsymbol{u}_{h}^{0}, \boldsymbol{d}_{h}^{0}\right) \in U_{0 h} \times D_{h}$ of $\left(\boldsymbol{u}_{0}, \boldsymbol{d}_{0}\right)$ :

Knowing $\left(\boldsymbol{u}_{h}^{n-1}, \boldsymbol{d}_{h}^{n-1}\right) \in U_{0 h} \times D_{h}$ and given an adequate approximation $\boldsymbol{l}_{h}^{n}$ of $\boldsymbol{l}\left(t_{n}\right)$, compute $\left(\boldsymbol{u}_{h}^{n}, \boldsymbol{w}_{h}^{n}\right) \in U_{0 h} \times W_{h}$ and $\left(p_{h}^{n}, \boldsymbol{d}_{h}^{n}\right) \in P_{0 h} \times D_{h}$ such that $\left.\boldsymbol{d}_{h}^{n}\right|_{\partial \Omega}=\boldsymbol{l}_{h}^{n}$ and:

$$
\begin{aligned}
& \left(\frac{1}{k}\left(\boldsymbol{u}_{h}^{n}-\boldsymbol{u}_{h}^{n-1}\right), \boldsymbol{v}\right)+\nu\left(\nabla \boldsymbol{u}_{h}^{n}, \nabla \boldsymbol{v}\right)-\left(p_{h}^{n}, \nabla \cdot \boldsymbol{v}\right) \\
& \quad+\left(\boldsymbol{u}_{h}^{n-1} \cdot \nabla \boldsymbol{u}_{h}^{n}+\frac{1}{2} \nabla \cdot \boldsymbol{u}_{h}^{n-1} \boldsymbol{u}_{h}^{n}, \boldsymbol{v}\right)-\mu\left(\left(\nabla \boldsymbol{d}_{h}^{n-1}\right)^{t} \boldsymbol{w}_{h}^{n}, \boldsymbol{v}\right)=0, \quad \forall \boldsymbol{v} \in U_{0 h}, \\
& \left(\frac{1}{k}\left(\boldsymbol{d}_{h}^{n}-\boldsymbol{d}_{h}^{n-1}\right), \boldsymbol{e}\right)+\left(\boldsymbol{u}_{h}^{n} \cdot \nabla \boldsymbol{d}_{h}^{n-1}, \boldsymbol{e}\right)+\frac{\gamma}{\mu}\left(\boldsymbol{w}_{h}^{n}, \boldsymbol{e}\right)=-\gamma\left(\boldsymbol{f}\left(\boldsymbol{d}_{h}^{n-1}\right), \boldsymbol{e}\right), \quad \forall \boldsymbol{e} \in W_{h}, \\
& \left(\nabla \cdot \boldsymbol{u}_{h}^{n}, q\right)=0, \quad \forall q \in P_{h}, \\
& \mu\left(\nabla \boldsymbol{d}_{h}^{n}, \nabla \boldsymbol{g}\right)-\left(\boldsymbol{w}_{h}^{n}, \boldsymbol{g}\right)=0, \quad \forall \boldsymbol{g} \in D_{0 h} .
\end{aligned}
$$

We shall prove that this linear semi-implicit scheme has the following properties:

(1) It generates a unique sequence of solutions $\left(\boldsymbol{u}_{h}^{n}, \boldsymbol{w}_{h}^{n}, p_{h}^{n}, \boldsymbol{d}_{h}^{n}\right)$.

(2) Under mild regularity assumptions on the data and if the above finite element spaces are constructed on a quasi-uniform (or uniformly regular) family of triangulations of $\bar{\Omega}$, this sequence converges in the appropriate spaces to a semi-strong solution $(\boldsymbol{u}, \boldsymbol{d})$ of problem (1.4)-(1.8) (see Definition 3.1).

(3) If this solution is sufficiently smooth, the error of this scheme measured in $L^{\infty}\left(\boldsymbol{L}^{2}(\Omega)\right) \cap L^{2}\left(\boldsymbol{H}^{1}(\Omega)\right)$ for the velocity and $L^{\infty}\left(\boldsymbol{H}^{1}(\Omega)\right)$ for the direction vector, is of order one in space and time (see Theorem 6.1).

Although the scheme is linearized, the simultaneous computation of all unknowns is impractical and, therefore, we present several convergent algorithms for decoupling the computation of $\left(\boldsymbol{u}_{h}, p_{h}\right)$ and $\left(\boldsymbol{w}_{h}, \boldsymbol{d}_{h}\right)$ at each time step. Knowing $\boldsymbol{w}_{h}^{n-1}$, $\boldsymbol{d}_{h}^{n-1}$ and $\boldsymbol{u}_{h}^{n-1}$, these algorithms approximate $\boldsymbol{w}_{h}^{n}, \boldsymbol{d}_{h}^{n}, \boldsymbol{u}_{h}^{n}$ and $p_{h}^{n}$ by an iterative method, computing the iterates $\left(\boldsymbol{u}_{i}, p_{i}\right)$ and $\left(\boldsymbol{w}_{i}, \boldsymbol{d}_{i}\right)$ by means of linear and decoupled problems. Here is one of them:

(1) Take $\boldsymbol{u}_{0}=\boldsymbol{u}_{h}^{n-1}$ and $\boldsymbol{w}_{0}=\boldsymbol{w}_{h}^{n-1}$. 
(2) Knowing $\boldsymbol{u}_{i-1}$ and $\boldsymbol{w}_{i-1}$, compute in parallel

- $\left(\boldsymbol{w}_{i}, \boldsymbol{d}_{i}\right) \in W_{h} \times D_{h}$, with $\left.\left(\boldsymbol{d}_{i}\right)\right|_{\partial \Omega}=\boldsymbol{l}_{h}^{n}$, solution of

$$
\begin{aligned}
& \frac{1}{k}\left(\boldsymbol{d}_{i}-\boldsymbol{d}_{h}^{n-1}, \boldsymbol{e}\right)+\frac{\gamma}{\mu}\left(\boldsymbol{w}_{i}, \boldsymbol{e}\right)=-\left(\boldsymbol{u}_{i-1} \cdot \nabla \boldsymbol{d}_{h}^{n-1}, \boldsymbol{e}\right)-\gamma\left(\boldsymbol{f}\left(\boldsymbol{d}_{h}^{n-1}\right), \boldsymbol{e}\right) \quad \forall \boldsymbol{e} \in W_{h}, \\
& \mu\left(\nabla \boldsymbol{d}_{i}, \nabla \boldsymbol{g}\right)-\left(\boldsymbol{w}_{i}, \boldsymbol{g}\right)=0 \quad \forall \boldsymbol{g} \in D_{0 h}, \\
& \quad \bullet\left(\boldsymbol{u}_{i}, p_{i}\right) \in U_{0 h} \times P_{h} \text { solution of } \\
& \frac{1}{k}\left(\boldsymbol{u}_{i}-\boldsymbol{u}_{h}^{n-1}, \boldsymbol{v}\right)+\nu\left(\nabla \boldsymbol{u}_{i}, \nabla \boldsymbol{v}\right)-\left(p_{i}, \nabla \cdot \boldsymbol{v}\right) \\
& =\mu\left(\left(\nabla \boldsymbol{d}_{h}^{n-1}\right)^{t} \boldsymbol{w}_{i-1}, \boldsymbol{v}\right)-\left(\boldsymbol{u}_{h}^{n-1} \cdot \nabla \boldsymbol{u}_{i-1}+\frac{1}{2} \nabla \cdot \boldsymbol{u}_{h}^{n-1} \boldsymbol{u}_{i-1}, \boldsymbol{v}\right) \quad \forall \boldsymbol{v} \in U_{0 h}, \\
& \left(\nabla \cdot \boldsymbol{u}_{i}, q\right)=0 \quad \forall q \in P_{h} .
\end{aligned}
$$

We shall prove that these algorithms converge geometrically to the desired solution.

Our work is organized as follows. In Section 2, we give a list of notation and some regularity results on general polyhedral domains that we shall need further on. In Section 3, we construct a suitable lifting of the boundary condition and we give the main ideas used in analyzing the continuous problem. Section 4 is devoted to the derivation of the mixed variational formulation. In Section 5, we define the fully discrete scheme and prove its stability and convergence. In Section 6, we establish a priori error estimates. Section 7 is devoted to the decoupling algorithms and their convergence.

\section{Preliminaires}

2.1. Notation. We shall use the following notation; for the sake of simplicity, we define them in three dimensions. Let $\left(k_{1}, k_{2}, k_{3}\right)$ denote a triple of non-negative integers, set $|k|=k_{1}+k_{2}+k_{3}$ and define the partial derivative $\partial^{k}$ by

$$
\partial^{k} v=\frac{\partial^{|k|} v}{\partial x_{1}^{k_{1}} \partial x_{2}^{k_{2}} \partial x_{3}^{k_{3}}} .
$$

Then, for any non-negative integer $m$ and number $r \geq 1$, recall the classical Sobolev space (cf. Adams [1] or Nečas [31])

$$
W^{m, r}(\Omega)=\left\{v \in L^{r}(\Omega) ; \partial^{k} v \in L^{r}(\Omega) \forall|k| \leq m\right\},
$$

equipped with the seminorm

$$
|v|_{W^{m, r}(\Omega)}=\left[\sum_{|k|=m} \int_{\Omega}\left|\partial^{k} v\right|^{r} d \boldsymbol{x}\right]^{1 / r},
$$

and norm (for which it is a Banach space)

$$
\|v\|_{W^{m, r}(\Omega)}=\left[\sum_{0 \leq|k| \leq m}|v|_{W^{k, r}(\Omega)}^{r}\right]^{1 / r},
$$

with the usual extension when $r=\infty$. The reader can refer to Lions \& Magenes [28] and [16] for extensions of this definition to non-integral values of $m$. When $r=2$, this space is the Hilbert space $H^{m}(\Omega)$. The definitions of these spaces are extended 
straightforwardly to vectors, with the same notation, but with the following modification for the norms in the non-Hilbert case. Let $\boldsymbol{u}=\left(\boldsymbol{u}_{1}, \boldsymbol{u}_{2}, \boldsymbol{u}_{3}\right)$; then we set

$$
\|\boldsymbol{u}\|_{L^{r}(\Omega)}=\left[\int_{\Omega}|\boldsymbol{u}(\boldsymbol{x})|^{r} d \boldsymbol{x}\right]^{1 / r},
$$

where $|\cdot|$ denotes the Euclidean vector norm for vectors or the Frobenius norm for tensors. As usual, $(\cdot, \cdot)$ denotes the $L^{2}(\Omega)$ scalar product.

When the boundary $\partial \Omega$ of $\Omega$ is a polygon or polyhedron, as will be the case in the sequel, the trace spaces $W^{s, r}(\partial \Omega)$ are defined as above for all $s \in[0,1]$ and $p \geq 1$. When $s>1$, the situation is more complex and to simplify, we define the space $W^{s, r}(\partial \Omega)$ as the trace space of all functions in $W^{s-1 / r, r}(\Omega)$. For instance, $H^{3 / 2}(\partial \Omega)$ is the trace space of $H^{2}(\Omega)$.

Let $\mathcal{D}(\Omega)$ denote the set of indefinitely differentiable functions with compact support in $\Omega$. For functions that vanish on the boundary, we define

$$
H_{0}^{1}(\Omega)=\left\{v \in H^{1}(\Omega) ;\left.v\right|_{\partial \Omega}=0\right\},
$$

and recall Poincaré's inequality: there exists a constant $C$ such that

$$
\|v\|_{L^{2}(\Omega)} \leq C \operatorname{diam}(\Omega)|v|_{H^{1}(\Omega)} \quad \forall v \in H_{0}^{1}(\Omega) .
$$

Owing to (2.10), we use the seminorm $|\cdot|_{H^{1}(\Omega)}$ as a norm on $H_{0}^{1}(\Omega)$.

We shall also use the standard spaces for incompressible fluids:

$$
\boldsymbol{H}=\left\{\boldsymbol{v} \in \boldsymbol{L}^{2}(\Omega) ; \nabla \cdot \boldsymbol{v}=0 \text { in } \Omega, \boldsymbol{v} \cdot \boldsymbol{n}=0 \text { on } \partial \Omega\right\},
$$

where $\boldsymbol{n}$ is the unit normal vector to $\partial \Omega$, exterior to $\Omega$,

$$
\begin{aligned}
\boldsymbol{V} & =\left\{\boldsymbol{v} \in \boldsymbol{H}_{0}^{1}(\Omega) ; \nabla \cdot \boldsymbol{v}=0 \text { in } \Omega\right\}, \\
L_{0}^{2}(\Omega) & =\left\{q \in L^{2}(\Omega) ; \int_{\Omega} q d \boldsymbol{x}=0\right\} .
\end{aligned}
$$

As usual, for handling time-dependent problems, it is convenient to consider functions defined on a time interval $] a, b[$ with values in a functional space, say $X$ (cf. [28]). More precisely, let $\|\cdot\|_{X}$ denote the norm of $X$; then for any number $r$, $1 \leq r \leq \infty$, we define

$$
L^{r}(a, b ; X)=\{f \text { measurable in }] a, b\left[; \int_{a}^{b}\|f(t)\|_{X}^{r} d t<\infty\right\}
$$

equipped with the norm

$$
\|f\|_{L^{r}(a, b ; X)}=\left(\int_{a}^{b}\|f(t)\|_{X}^{r} d t\right)^{1 / r},
$$

with the usual modification if $r=\infty$. It is a Banach space if $X$ is a Banach space. When $r=2, L^{2}\left(a, b ; H^{m}(\Omega)\right)$ is a Hilbert space and, in particular, $L^{2}\left(a, b ; L^{2}(\Omega)\right)$ coincides with $L^{2}(\Omega \times] a, b[)$. We shall also use spaces with derivatives in time, such as

$$
H^{1}(a, b ; X)=\left\{f \in L^{2}(] a, b[; X) ; \frac{\partial f}{\partial t} \in L^{2}(] a, b[; X)\right\},
$$

equipped with the graph norm

$$
\|f\|_{H^{1}(a, b ; X)}=\left(\|f\|_{L^{2}(a, b ; X)}^{2}+\left\|\frac{\partial f}{\partial t}\right\|_{L^{2}(a, b ; X)}^{2}\right)^{1 / 2},
$$


for which is a Hilbert space. To simplify, we shall use the letter $Q$ for the space-time cylinder $\Omega \times] 0, T\left[\right.$ and denote $L^{p}(0, T ; X)$ by $L^{p}(X)$, etc.

2.2. Auxiliary regularity results. Let us start with the solution $v$ of the Laplace equation

$$
-\Delta v=f \text { in } \Omega,\left.v\right|_{\partial \Omega}=g .
$$

When $(f, g)$ belongs to $H^{-1}(\Omega) \times H^{1 / 2}(\partial \Omega)$, this problem has a unique solution $v$ in $H^{1}(\Omega)$. When $\Omega$ has a $\mathcal{C}^{1,1}$ boundary or is convex and $(f, g)$ belongs to $L^{2}(\Omega) \times H^{3 / 2}(\partial \Omega)$, then $v$ belongs to $H^{2}(\Omega)$, with continuous dependence on $f$ and $g$ (cf. [16]). When $\Omega$ is a polygon or polyhedron, without restricting the angles of $\partial \Omega, v$ has the following regularity; the first theorem is due to [16] and the second one to 12 .

Theorem 2.1. Let $\Omega$ be a polygon in two dimensions. If $(f, g)$ belongs to $L^{r}(\Omega) \times$ $W^{2-1 / r, r}(\partial \Omega)$ for some $r$ with $1<r \leq 4 / 3$, then the solution $v$ of (2.11) belongs to $W^{2, r}(\Omega)$ with continuous dependence on $f$ and $g$.

Theorem 2.2. Let $\Omega$ be a polyhedron with a Lipschitz-continuous boundary or a polygon. If $(f, g)$ belongs to $H^{s-1}(\Omega) \times H^{s+1 / 2}(\partial \Omega)$ for some $s$ with $0 \leq s<1 / 2$, then the solution $v$ of (2.11) belongs to $H^{s+1}(\Omega)$ with continuous dependence on $f$ and $g$.

The first part of the next result for the borderline case $s=1 / 2$ is due to Jerrison \& Kenig [21] and the second part is established by Dauge \& Costabel for the Stokes problem and is written in Girault \& Lions [14, but the argument carries over to the Laplace equation. Both proofs are written in three dimensions, but their conclusions are valid in two dimensions.

Theorem 2.3. Let $\Omega$ be a Lipschitz domain of $\mathbb{R}^{2}$ or $\mathbb{R}^{3}$. If $f=0$ and $g$ belongs to $H^{1}(\partial \Omega)$, then the solution $v$ of (2.11) belongs to $H^{3 / 2}(\Omega)$ with continuous dependence on $\mathrm{g}$.

When $\Omega$ is a polyhedron with a Lipschitz-continuous boundary or a polygon, $g$ belongs to $H^{1}(\partial \Omega)$ and $f$ belongs to $L^{3 / 2}(\Omega)$, then the solution $v$ of (2.11) belongs to $H^{3 / 2}(\Omega)$ with continuous dependence on $f$ and $g$.

We have similar regularity results for the solution $(\boldsymbol{v}, q)$ of the Stokes problem in a connected Lipschitz domain:

$$
-\Delta \boldsymbol{v}+\nabla q=\boldsymbol{f}, \nabla \cdot \boldsymbol{v}=0 \text { in } \Omega,\left.\boldsymbol{v}\right|_{\partial \Omega}=\mathbf{0} .
$$

When $\boldsymbol{f}$ belongs to $\boldsymbol{H}^{-1}(\Omega),(2.12)$ has a unique solution $(\boldsymbol{v}, q)$ in $\boldsymbol{V} \times L_{0}^{2}(\Omega)$ that depends continuously on $\boldsymbol{f}$. When $\boldsymbol{f}$ belongs to $\boldsymbol{L}^{2}(\Omega)$ and the domain has a $\mathcal{C}^{1,1}$ boundary or is a convex polygon (cf. Kellog \& Osborn [22] or [16]) or polyhedron (cf. [13), then the solution $(\boldsymbol{v}, q)$ of (2.12) belongs to $\boldsymbol{H}^{2}(\Omega) \times H^{1}(\Omega)$, with continuous dependence on $\boldsymbol{f}$. Without restriction on the angles of $\partial \Omega$, the following theorems hold; the first one can be found in [16] and the second one in [13.

Theorem 2.4. Let $\Omega$ be a connected polygon in two dimensions. If $\boldsymbol{f}$ belongs to $\boldsymbol{L}^{r}(\Omega)$ for some $r$ with $1<r \leq 4 / 3$, then the solution $(\boldsymbol{v}, q)$ of (2.12) belongs to $\boldsymbol{W}^{2, r}(\Omega) \times W^{1, r}(\Omega)$ with continuous dependence on $\boldsymbol{f}$. 
Theorem 2.5. Let $\Omega$ be a connected polyhedron with a Lipschitz-continuous boundary or a polygon. If $\boldsymbol{f}$ belongs to $\boldsymbol{H}^{s-1}(\Omega)$ for some $s$ with $0 \leq s<1 / 2$, then the solution $(\boldsymbol{v}, q)$ of $(2.12)$ belongs to $\boldsymbol{H}^{s+1}(\Omega) \times H^{s}(\Omega)$ with continuous dependence on $f$.

As mentioned above, the following result for the borderline case $s=1 / 2$ is due to Dauge \& Costabel and written in [14]:

Theorem 2.6. Let $\Omega$ be a connected polyhedron with a Lipschitz-continuous boundary or a polygon. If $\boldsymbol{f}$ belongs to $\boldsymbol{L}^{3 / 2}(\Omega)$, then the solution $(\boldsymbol{v}, q)$ of (2.12) belongs to $\boldsymbol{H}^{3 / 2}(\Omega) \times H^{1 / 2}(\Omega)$ with continuous dependence on $\boldsymbol{f}$.

\section{The EXACT PROBLEM}

Since our primary purpose is numerical approximation, we suppose here that the domain $\Omega$ is a connected polygon or Lipschitz polyhedron. In addition, to simplify the discussion, we write the analysis for the three-dimensional case. All results extend easily, with simpler proofs, to plane domains.

3.1. The problem in a semi-variational form. To begin with, let us put (1.5) into a weak variational form. By taking formally the scalar product of both sides of (1.5) with a test function $\boldsymbol{v}$ in $\boldsymbol{V}$, applying Green's formula and the identity

$$
\nabla \cdot(\nabla \boldsymbol{d} \odot \nabla \boldsymbol{d})=\frac{1}{2} \nabla\left(|\nabla \boldsymbol{d}|^{2}\right)+(\nabla \boldsymbol{d})^{t} \Delta \boldsymbol{d}
$$

we obtain

$$
d_{t}(\boldsymbol{u}, \boldsymbol{v})+\nu(\nabla \boldsymbol{u}, \nabla \boldsymbol{v})+(\boldsymbol{u} \cdot \nabla \boldsymbol{u}, \boldsymbol{v})+\lambda\left((\nabla \boldsymbol{d})^{t} \Delta \boldsymbol{d}, \boldsymbol{v}\right)=0 .
$$

We shall see further on that a similar variational formulation is not needed for (1.4). This leads us to consider a "semi-strong" solution $(\boldsymbol{u}, \boldsymbol{d})$ of the penalized problem (1.4) - (1.8), verifying the system (1.5) for $\boldsymbol{u}$ in the sense of distributions and the system (1.4) for $\boldsymbol{d}$ pointwise a.e. in $Q$. To be specific, we introduce the following:

Definition 3.1. Let $\boldsymbol{l}$ be given in $H^{1}\left(0, T ; \boldsymbol{H}^{1}(\partial \Omega)\right)$ and $\left(\boldsymbol{u}_{0}, \boldsymbol{d}_{0}\right)$ in $\boldsymbol{H} \times \boldsymbol{H}^{1}(\Omega)$ verifying the compatibility condition $\boldsymbol{l}(0)=\left.\boldsymbol{d}_{0}\right|_{\partial \Omega}$. A pair $(\boldsymbol{u}, \boldsymbol{d})$ is called a "semistrong" solution in $] 0, T[$ of problem (1.4)-(1.8) if

$$
\begin{gathered}
\boldsymbol{u} \in L^{2}(\boldsymbol{V}) \cap L^{\infty}(\boldsymbol{H}), \\
\boldsymbol{d} \in L^{\infty}\left(\boldsymbol{H}^{1}(\Omega)\right), \Delta \boldsymbol{d} \in L^{2}\left(\boldsymbol{L}^{2}(\Omega)\right),\left.\quad \boldsymbol{d}\right|_{\partial \Omega \times] 0, T[}=\boldsymbol{l},
\end{gathered}
$$

verify the equations

$$
\begin{aligned}
d_{t}(\boldsymbol{u}, \boldsymbol{v})+\nu(\nabla \boldsymbol{u}, \nabla \boldsymbol{v})+(\boldsymbol{u} \cdot \nabla \boldsymbol{u}, \boldsymbol{v})+\lambda\left((\nabla \boldsymbol{d})^{t} \Delta \boldsymbol{d}, \boldsymbol{v}\right) & =0, \quad \forall \boldsymbol{v} \in \boldsymbol{V} \\
\partial_{t} \boldsymbol{d}+\boldsymbol{u} \cdot \nabla \boldsymbol{d}+\gamma(\boldsymbol{f}(\boldsymbol{d})-\Delta \boldsymbol{d}) & =\mathbf{0}
\end{aligned}
$$

and the initial conditions

$$
\left.\boldsymbol{u}\right|_{t=0}=\left.\boldsymbol{u}_{0} \quad \boldsymbol{d}\right|_{t=0}=\boldsymbol{d}_{0} .
$$

Remark 3.2. Since $\Omega$ is a Lipschitz polyhedron, the assumption on $\boldsymbol{l}$ and Theorem 2.3 imply that $\boldsymbol{d}$ belongs to $L^{2}\left(\boldsymbol{H}^{3 / 2}(\Omega)\right)$. Therefore all terms in (3.4) are well defined. Of course, when $\boldsymbol{l} \in H^{1}\left(0, T ; \boldsymbol{H}^{3 / 2}(\partial \Omega)\right)$ and $\Omega$ is convex or its boundary is $\mathcal{C}^{1,1}$, then (3.3) yields that $\boldsymbol{d}$ belongs to $L^{2}\left(\boldsymbol{H}^{2}(\Omega)\right)$. 
Remark 3.3. The functions $\boldsymbol{u}$ and $\boldsymbol{d}$ are continuous in time, owing to the additional regularity

$$
\partial_{t} \boldsymbol{u} \in L^{1}\left(\boldsymbol{V}^{\prime}\right) \quad \text { and } \quad \partial_{t} \boldsymbol{d} \in L^{2}\left(\boldsymbol{L}^{3 / 2}(\Omega)\right)
$$

that can be established applying (3.2) and (3.3) to (3.4) and (3.5), respectively. Therefore, the initial conditions (3.6) make sense.

System (3.2)-(3.6) is equivalent to the original problem (1.4)-(1.8) in the following sense: If $(\boldsymbol{u}, \boldsymbol{d})$ satisfies (3.2)-(3.6), then as for the Navier-Stokes equations, there exists a pressure $\tilde{p}$ in $H^{-1}\left(0, T ; L_{0}^{2}(\Omega)\right)$ (at least) such that

$$
\partial_{t} \boldsymbol{u}+\boldsymbol{u} \cdot \nabla \boldsymbol{u}-\nu \Delta \boldsymbol{u}+\nabla \tilde{p}+\lambda(\nabla \boldsymbol{d})^{t} \Delta \boldsymbol{d}=\mathbf{0} .
$$

In view of (3.1) and setting

$$
p=\tilde{p}-\frac{1}{2}|\nabla \boldsymbol{d}|^{2},
$$

we recover (1.5). To simplify the discussion, we drop the tilde and denote $p$ and $\tilde{p}$ by the same symbol $p$. Conversely, any solution of (1.4) -1.8) with the above regularity solves (3.4)-(3.6).

3.2. Existence of solutions. The problem for $\boldsymbol{d}$ (given $\boldsymbol{u}$ ) satisfies the following crucial maximum principle; see [24, 8, 18.

Lemma 3.4. Let $\boldsymbol{u}$ be given in $L^{2}(\boldsymbol{V})$; let $|\boldsymbol{l}(t, \boldsymbol{x})| \leq 1$ a.e. on $\left.\partial \Omega \times\right] 0, T[$ and $\left|\boldsymbol{d}_{0}(\boldsymbol{x})\right| \leq 1$ a.e. in $\Omega$ and let $\boldsymbol{d}$ be a "strong" solution in $] 0, T[$ of problem (3.3), (3.5) and the second part of (3.6). Then $\boldsymbol{d}$ verifies $|\boldsymbol{d}(t, \boldsymbol{x})| \leq 1$ a.e. in $Q$.

Proof. Let us sketch the main points in the proof; a complete derivation can be found in the references above. Multiplying (3.5) by $\boldsymbol{d}$, applying the identity

$$
\Delta(\boldsymbol{d} \cdot \boldsymbol{d})=2 \Delta \boldsymbol{d} \cdot \boldsymbol{d}+2(\nabla \boldsymbol{d}) \cdot(\nabla \boldsymbol{d}),
$$

and using the positivity of $(\nabla \boldsymbol{d}) \cdot(\nabla \boldsymbol{d})=|\nabla \boldsymbol{d}|^{2}$, we obtain

$$
\partial_{t}\left(|\boldsymbol{d}|^{2}-1\right)+2 \gamma \boldsymbol{f}(\boldsymbol{d}) \cdot \boldsymbol{d}-\gamma \Delta\left(|\boldsymbol{d}|^{2}-1\right)+(\boldsymbol{u} \cdot \nabla)\left(|\boldsymbol{d}|^{2}-1\right) \leq 0 \quad \text { a.e. in } Q .
$$

Setting

$$
\psi(\boldsymbol{d})=\left(|\boldsymbol{d}|^{2}-1\right)_{+},
$$

which belongs to $H_{0}^{1}(\Omega)$, observing that

$$
\boldsymbol{f}(\boldsymbol{d}) \cdot \boldsymbol{d}\left(|\boldsymbol{d}|^{2}-1\right)_{+} \geq 0 \text { and that } \int_{\Omega}(\boldsymbol{u} \cdot \nabla)\left(|\boldsymbol{d}|^{2}-1\right) \psi(\boldsymbol{d}) d \boldsymbol{x}=0,
$$

and taking the inner product of (3.7) in $\Omega$ with $\psi(\boldsymbol{d})$, we obtain formally the inequality:

$$
\left.\frac{d}{d t}\|\psi(\boldsymbol{d})\|_{L^{2}(\Omega)}^{2}+2 \gamma\|\nabla \psi(\boldsymbol{d})\|_{L^{2}(\Omega)}^{2} \leq 0 \quad \text { a.e. in }\right] 0, T[.
$$

Together with the fact that $\psi\left(\boldsymbol{d}_{0}\right)=0$ in $\Omega$, this implies that $\psi(\boldsymbol{d})=0$ a.e. in $Q$, and therefore $|\boldsymbol{d}| \leq 1$ in $Q$.

The proof of existence of solutions is based on this principle. It is used by 24] in the case where $\boldsymbol{l}$ is independent of time (i.e. $\boldsymbol{l}=\boldsymbol{l}(\boldsymbol{x})$ ) and by [8] in the case of time-periodic solutions. To handle theoretically the variable non-homogeneous boundary condition, we propose an adequate lifting similar to that used in [8]: For any $t$ a.e. in $] 0, T$, we define $\widetilde{\boldsymbol{d}}(t)$ as the solution of the elliptic problem

$$
-\Delta \widetilde{\boldsymbol{d}}(t)=\mathbf{0} \text { in } \Omega,\left.\quad \tilde{\boldsymbol{d}}(t)\right|_{\partial \Omega}=\boldsymbol{l}(t) \text { on } \partial \Omega,
$$


and we set

$$
\widehat{\boldsymbol{d}}=\boldsymbol{d}-\tilde{\boldsymbol{d}}
$$

Clearly, as $\widetilde{\boldsymbol{d}}$ is known, finding $\boldsymbol{d}$ is equivalent to finding $\widehat{\boldsymbol{d}}$. It stems from Theorem 2.3 that $\tilde{\boldsymbol{d}} \in H^{1}\left(0, T ; \boldsymbol{H}^{3 / 2}(\Omega)\right)$, since $\boldsymbol{l} \in H^{1}\left(0, T ; \boldsymbol{H}^{1}(\partial \Omega)\right)$. In particular, $\widehat{\boldsymbol{d}} \in$ $L^{\infty}\left(\boldsymbol{H}^{1}(\Omega)\right) \cap L^{2}\left(\boldsymbol{H}^{3 / 2}(\Omega)\right)$ is the solution of the elliptic problem $\Delta \widehat{\boldsymbol{d}}=\Delta \boldsymbol{d}$ and $\widehat{\boldsymbol{d}}_{\mid \partial \Omega}=\mathbf{0}$.

Remark 3.5. The regularity assumption for $\boldsymbol{l}$ can be relaxed. Indeed, in what follows, we only need that

$$
\widetilde{\boldsymbol{d}} \in L^{\infty}\left(\boldsymbol{H}^{1}(\Omega)\right), \quad \partial_{t} \widetilde{\boldsymbol{d}} \in L^{2}\left(\boldsymbol{L}^{2}(\Omega)\right) .
$$

With this lifting, we can prove the following existence result:

Theorem 3.6. Let $T>0$ and $\Omega$ be an open, bounded, connected Lipschitz polyhedron. For given $\boldsymbol{u}_{0} \in \boldsymbol{H}, \boldsymbol{d}_{0} \in \boldsymbol{H}^{1}$ and $\boldsymbol{l} \in H^{1}\left(0, T ; \boldsymbol{H}^{1}(\partial \Omega)\right)$ such that $\boldsymbol{l}(0)=\boldsymbol{d}_{0}$ a.e. on $\partial \Omega,\left|\boldsymbol{d}_{0}(\boldsymbol{x})\right| \leq 1$ a.e. in $\Omega$ and $|\boldsymbol{l}(t, \boldsymbol{x})| \leq 1$ a.e. in $\left.\partial \Omega \times\right] 0, T[$, the coupled Navier-Stokes and Ginzburg-Landau model (1.4)-(1.8) has a global "semi-strong" solution $(\boldsymbol{u}, \boldsymbol{d})$.

Proof. Let us sketch the proof; several arguments can already be found in [24] and 8]. We proceed in four steps.

i) A priori energy equality. Assume that (1.4) (1.8) has a sufficiently smooth solution $(\boldsymbol{u}, \boldsymbol{d})$. Using the lifting function $\widetilde{\boldsymbol{d}}$, taking into account that $\Delta \boldsymbol{d}=\Delta \widehat{\boldsymbol{d}}$, choosing $\boldsymbol{v}=\boldsymbol{u}$ in (3.4), multiplying (3.5) by $\lambda(\boldsymbol{f}(\boldsymbol{d})-\Delta \boldsymbol{d}$ ), adding the resulting equations, and applying twice the relation (1.3), we obtain the a priori energy equality, as in [8]:

$$
\begin{aligned}
\frac{d}{d t}\left(\frac{1}{2}\|\boldsymbol{u}\|_{L^{2}(\Omega)}^{2}+\right. & \left.\frac{\lambda}{2}\|\nabla \widehat{\boldsymbol{d}}\|_{L^{2}(\Omega)}^{2}+\lambda \int_{\Omega} F(\boldsymbol{d}) d \boldsymbol{x}\right)+\nu\|\nabla \boldsymbol{u}\|_{L^{2}(\Omega)}^{2} \\
& +\lambda \gamma\|\boldsymbol{f}(\boldsymbol{d})-\Delta \boldsymbol{d}\|_{L^{2}(\Omega)}^{2}=\lambda \int_{\Omega} \partial_{t} \tilde{\boldsymbol{d}} \cdot \Delta \boldsymbol{d} d \boldsymbol{x} .
\end{aligned}
$$

In 24, where $\boldsymbol{l}=\boldsymbol{l}(\boldsymbol{x})$ does not depend on time, a similar equality is derived with $\boldsymbol{d}$ instead of $\widehat{\boldsymbol{d}}$ and right-hand side zero. This is possible because, in this case, Green's formula yields

$$
-\int_{\Omega}\left(\partial_{t} \boldsymbol{d}\right) \Delta \boldsymbol{d} d \boldsymbol{x}=\frac{1}{2} \frac{d}{d t} \int_{\Omega}|\nabla \boldsymbol{d}|^{2} d \boldsymbol{x} .
$$

ii) Construction of a semi-discrete Galerkin solution. Let $\left(\boldsymbol{u}_{m}, \boldsymbol{d}_{m}=\widehat{\boldsymbol{d}}_{m}+\widetilde{\boldsymbol{d}}\right)$ be an approximate solution defined by a semi-Galerkin method, where (3.4) is discretized in a suitable finite-dimensional subspace $\boldsymbol{V}_{m}$ of $\boldsymbol{V}$ and (3.5) is solved exactly for $\boldsymbol{d}_{m}$, knowing $\boldsymbol{u}_{m}$. Existence (and uniqueness) of such approximate solutions is not straightforward, because a fixed-point operator $R: \boldsymbol{v} \rightarrow \boldsymbol{d} \rightarrow \boldsymbol{u}$ is constructed by formulating decoupled problems, first for $\boldsymbol{d}$ and next for $\boldsymbol{u}$. Then, the weak estimates for $\boldsymbol{u}$ depend on strong estimates for $\boldsymbol{d}$, and these in turn depend on weak estimates for $\boldsymbol{v}$. The argument in 24] consists in obtaining local in time approximate solutions (that are fixed points of the above operator $R$ ) and extending them by a continuation argument that allows to reach any time, by progressing on small time intervals of fixed length.

iii) A priori estimates for the semi-discrete Galerkin solution. Since $\boldsymbol{d}_{m}$ solves exactly (3.5) with $\boldsymbol{u}=\boldsymbol{u}_{m} \in \boldsymbol{V}$, then the maximum principle given in Lemma 3.4 
implies that

$$
\left|\boldsymbol{d}_{m}\right| \leq 1 \quad \text { a.e. in } Q \text {. }
$$

On the other hand, it is easy to check that the pair $\left(\boldsymbol{u}_{m}, \boldsymbol{d}_{m}\right)$ satisfies the energy equality (3.9). Therefore, using (3.10) and (1.2), we derive from (3.9) that

$$
\begin{array}{rll}
\boldsymbol{u}_{m} & \text { is bounded in } & L^{\infty}(\boldsymbol{H}) \cap L^{2}(\boldsymbol{V}), \\
\widehat{\boldsymbol{d}}_{m} & \text { is bounded in } & L^{\infty}\left(\boldsymbol{H}^{1}(\Omega)\right), \\
\Delta \widehat{\boldsymbol{d}}_{m} & \text { is bounded in } & L^{2}\left(\boldsymbol{L}^{2}(\Omega)\right) .
\end{array}
$$

Then, by virtue of Theorem 2.3, each coefficient of $\nabla \widehat{\boldsymbol{d}}_{m}$ is bounded in $L^{2}\left(H^{1 / 2}(\Omega)\right)$. Moreover, substituting estimates (3.11)-(3.13) into the equations (3.4)-(3.5) for $\left(\boldsymbol{u}_{m}, \boldsymbol{d}_{m}\right)$ and using (3.1), we obtain

$$
\begin{array}{lll}
\partial_{t} \boldsymbol{u}_{m} & \text { is bounded in } & L^{1}\left(\boldsymbol{V}^{\prime}\right), \\
\partial_{t} \widehat{\boldsymbol{d}}_{m} & \text { is bounded in } & L^{2}\left(\boldsymbol{L}^{3 / 2}\right) .
\end{array}
$$

iv) Compactness and passing to the limit. It follows from (3.14), (3.15) and [36, that $\boldsymbol{u}_{m}$ and $\nabla \widehat{\boldsymbol{d}}_{m}$ are compact in $L^{2}\left(\boldsymbol{L}^{2}\right)$. Then a standard argument permits us to pass to the limit as $m \rightarrow \infty$ in the non-linear terms of (3.4)-(3.5) for $\left(\boldsymbol{u}_{m}, \boldsymbol{d}_{m}\right)$, thus yielding existence of a solution.

3.3. An equivalent problem. The maximum principle in Lemma 3.4 suggests to truncate the penalty function $\boldsymbol{f}$, i.e., define $\widetilde{\boldsymbol{f}}$ by

$$
\widetilde{\boldsymbol{f}}(\boldsymbol{d})=-\frac{1}{\varepsilon^{2}}\left(1-|\boldsymbol{d}|^{2}\right)_{+} \quad \text { i.e. } \tilde{\boldsymbol{f}}(\boldsymbol{d})= \begin{cases}\boldsymbol{f}(\boldsymbol{d}) & \text { if }|\boldsymbol{d}| \leq 1, \\ \mathbf{0} & \text { otherwise. }\end{cases}
$$

Observe that $\widetilde{\boldsymbol{f}}(\boldsymbol{d})=\boldsymbol{f}(T(\boldsymbol{d}))$, where

$$
T(\boldsymbol{d})= \begin{cases}\boldsymbol{d} & \text { if }|\boldsymbol{d}| \leq 1, \\ \frac{\boldsymbol{d}}{|\boldsymbol{d}|} & \text { otherwise }\end{cases}
$$

and $\widetilde{\boldsymbol{f}}$ has the potential function $\widetilde{F}$ :

$$
\forall \boldsymbol{d} \in \mathbb{R}^{N}, \widetilde{\boldsymbol{f}}(\boldsymbol{d})=\nabla_{\boldsymbol{d}}(\widetilde{F}(\boldsymbol{d})) \text {, where } \widetilde{F}(\boldsymbol{d})=\frac{1}{4 \varepsilon^{2}}\left[\left(1-|\boldsymbol{d}|^{2}\right)_{+}\right]^{2} .
$$

Hence, we replace $\boldsymbol{f}$ by $\widetilde{\boldsymbol{f}}$ in (3.5) and it is easy to check that this leads to a problem equivalent to (3.2)-(3.6), whenever the assumptions of Lemma 3.4 hold.

Proposition 3.7. Let $|\boldsymbol{l}(t, \boldsymbol{x})| \leq 1$ a.e. on $\partial \Omega \times] 0, T\left[\right.$ and $\left|\boldsymbol{d}_{0}(\boldsymbol{x})\right| \leq 1$ a.e. in $\Omega$. Then $(\boldsymbol{u}, \boldsymbol{d})$ is a semi-strong solution of (3.2)-(3.6) if and only if it is a semi-strong solution of the same problem with (3.5) replaced by

$$
\partial_{t} \boldsymbol{d}+\boldsymbol{u} \cdot \nabla \boldsymbol{d}+\gamma(\tilde{\boldsymbol{f}}(\boldsymbol{d})-\Delta \boldsymbol{d})=\mathbf{0} .
$$

From now on, we shall replace $\boldsymbol{f}$ by the truncated function $\tilde{\boldsymbol{f}}$; in particular, we shall use the bound

$$
\forall \boldsymbol{d} \in \mathbb{R}^{N},|\widetilde{\boldsymbol{f}}(\boldsymbol{d})| \leq \varepsilon^{-2},
$$

and refer to the Appendix for other properties of $\tilde{\boldsymbol{f}}$. 


\section{A MiXed FORMUlation With AN AUXiLiaRy VARIABle}

In order to eliminate $\Delta \boldsymbol{d}$ in (3.4) and (3.5), we define the auxiliary variable:

$$
\boldsymbol{w}=-\sqrt{\lambda} \Delta \boldsymbol{d}=-\mu \Delta \boldsymbol{d}=-\mu \Delta \widehat{\boldsymbol{d}} \quad \text { where } \mu=\sqrt{\lambda} .
$$

Considering that $\widehat{\boldsymbol{d}}$ vanishes on $\partial \Omega$, the relation between $\widehat{\boldsymbol{d}}$ and $\boldsymbol{w}$ is expressed in variational form through:

$$
\forall \boldsymbol{g} \in \boldsymbol{H}_{0}^{1}(\Omega), \mu(\nabla \widehat{\boldsymbol{d}}, \nabla \boldsymbol{g})-(\boldsymbol{w}, \boldsymbol{g})=0 .
$$

Thus, each $\boldsymbol{w}$ in $\boldsymbol{L}^{2}(\Omega)$ (and even in $\boldsymbol{H}^{-1}(\Omega)$ ) defines a unique $\widehat{\boldsymbol{d}}$ in $\boldsymbol{H}_{0}^{1}(\Omega)$ and we have the isometries:

$$
\|\boldsymbol{w}\|_{H^{-1}(\Omega)}=\mu\|\nabla \widehat{\boldsymbol{d}}\|_{L^{2}(\Omega)} \quad \text { and } \quad\|\boldsymbol{w}\|_{L^{2}(\Omega)}=\mu\|\Delta \widehat{\boldsymbol{d}}\|_{L^{2}(\Omega)} .
$$

Then, recovering the pressure $p$, we can replace (3.4) and (3.5) by the equivalent formulation

$$
\begin{aligned}
\forall \boldsymbol{v} \in \boldsymbol{H}_{0}^{1}(\Omega), d_{t}(\boldsymbol{u}, \boldsymbol{v})+\nu(\nabla \boldsymbol{u}, \nabla \boldsymbol{v})+(\boldsymbol{u} \cdot \nabla \boldsymbol{u}, \boldsymbol{v}) & -\mu\left((\nabla \boldsymbol{d})^{t} \boldsymbol{w}, \boldsymbol{v}\right)-(p, \nabla \cdot \boldsymbol{v})=0, \\
\forall \boldsymbol{e} \in \boldsymbol{L}^{2}(\Omega), \gamma(\boldsymbol{w}, \boldsymbol{e})+\mu\left\{d_{t}(\boldsymbol{d}, \boldsymbol{e})+(\boldsymbol{u} \cdot \nabla \boldsymbol{d}, \boldsymbol{e})+\gamma(\tilde{\boldsymbol{f}}(\boldsymbol{d}), \boldsymbol{e})\right\} & =0, \\
\forall q \in L_{0}^{2}(\Omega),(\nabla \cdot \boldsymbol{u}, q) & =0, \\
\forall \boldsymbol{g} \in \boldsymbol{H}_{0}^{1}(\Omega), \mu(\nabla \widehat{\boldsymbol{d}}, \nabla \boldsymbol{g})-(\boldsymbol{w}, \boldsymbol{g}) & =0,
\end{aligned}
$$

where $\boldsymbol{d}=\widehat{\boldsymbol{d}}+\widetilde{\boldsymbol{d}}, \tilde{\boldsymbol{d}}$ solves (3.8), $u \in L^{\infty}(\boldsymbol{H}) \cap L^{2}(\boldsymbol{V}), \boldsymbol{d} \in L^{\infty}\left(\boldsymbol{H}^{1}(\Omega)\right) \cap$ $L^{2}\left(\boldsymbol{H}^{3 / 2}(\Omega)\right), \boldsymbol{w} \in L^{2}\left(\boldsymbol{L}^{2}(\Omega)\right)$, and $p \in H^{-1}\left(0, T ; L_{0}^{2}(\Omega)\right)$. Since $H^{3 / 2}(\Omega)$ is embedded into $W^{1,3}(\Omega)$, all terms in the first two lines of (4.2) are well defined.

As the last line of (4.2) defines $\widehat{\boldsymbol{d}}$ (and hence $\boldsymbol{d}$ ) in terms of $\boldsymbol{w}$, we can swap $\boldsymbol{w}$ and $\boldsymbol{d}$ and consider that $\boldsymbol{w}$ is the main variable while $\boldsymbol{d}$ is the auxiliary variable that can be computed from $\boldsymbol{w}$. Hence, if $\boldsymbol{w}$ belongs to $L^{2}\left(\boldsymbol{L}^{2}(\Omega)\right)$, it follows from Theorem 2.3 that $\widehat{\boldsymbol{d}}$ belongs to $L^{2}\left(\boldsymbol{H}^{3 / 2}(\Omega)\right)$. With this idea in mind, let us set (4.2) into a mixed framework, introducing the following bilinear and trilinear continuous forms:

$$
\begin{aligned}
& a_{1}(\boldsymbol{u}, \boldsymbol{v})=\nu(\nabla \boldsymbol{u}, \nabla \boldsymbol{v}) \quad \forall \boldsymbol{u}, \boldsymbol{v} \in \boldsymbol{H}^{1}(\Omega), \\
& b(\boldsymbol{u}, q)=-(\nabla \cdot \boldsymbol{u}, q) \quad \forall \boldsymbol{u} \in \boldsymbol{H}^{1}(\Omega), q \in L^{2}(\Omega), \\
& c_{1}(\overline{\boldsymbol{u}}, \boldsymbol{u}, \boldsymbol{v})=(\overline{\boldsymbol{u}} \cdot \nabla \boldsymbol{u}, \boldsymbol{v}) \quad \forall \overline{\boldsymbol{u}}, \boldsymbol{u}, \boldsymbol{v} \in \boldsymbol{H}^{1}(\Omega) .
\end{aligned}
$$

On one hand, the identity

$$
\forall \boldsymbol{d}, \boldsymbol{v}, \boldsymbol{w} \in \mathbb{R}^{N},(\nabla \boldsymbol{d})^{t} \boldsymbol{w} \cdot \boldsymbol{v}=(\boldsymbol{v} \cdot \nabla) \boldsymbol{d} \cdot \boldsymbol{w},
$$

implies

$$
\forall \boldsymbol{d} \in \boldsymbol{W}^{1,3}(\Omega), \boldsymbol{w} \in \boldsymbol{L}^{2}(\Omega), \boldsymbol{v} \in \boldsymbol{H}^{1}(\Omega),\left((\nabla \boldsymbol{d})^{t} \boldsymbol{w}, \boldsymbol{v}\right)=c_{1}(\boldsymbol{v}, \boldsymbol{d}, \boldsymbol{w}) .
$$

On the other hand, in order to enforce the well-known antisymmetry of $c_{1}(\overline{\boldsymbol{u}}, \cdot \cdot \cdot)$ :

$$
\forall \bar{u} \in \boldsymbol{V}, \forall \boldsymbol{u}, \boldsymbol{v} \in \boldsymbol{H}_{0}^{1}(\Omega), c_{1}(\overline{\boldsymbol{u}}, \boldsymbol{u}, \boldsymbol{v})=-c_{1}(\overline{\boldsymbol{u}}, \boldsymbol{v}, \boldsymbol{u}),
$$


that may not necessarily hold when $\boldsymbol{V}$ is discretized, we replace $c_{1}(\overline{\boldsymbol{u}}, \boldsymbol{u}, \boldsymbol{v})$ by

$$
\widetilde{c_{1}}(\overline{\boldsymbol{u}}, \boldsymbol{u}, \boldsymbol{v}):=\frac{1}{2}\left\{c_{1}(\overline{\boldsymbol{u}}, \boldsymbol{u}, \boldsymbol{v})-c_{1}(\overline{\boldsymbol{u}}, \boldsymbol{v}, \boldsymbol{u})\right\}=c_{1}(\overline{\boldsymbol{u}}, \boldsymbol{u}, \boldsymbol{v})+\frac{1}{2}(\nabla \cdot \overline{\boldsymbol{u}}, \boldsymbol{u} \cdot \boldsymbol{v}) .
$$

Then the first two lines of (4.2) have the form

$$
\begin{gathered}
d_{t}(\boldsymbol{u}, \boldsymbol{v})+a_{1}(\boldsymbol{u}, \boldsymbol{v})+\widetilde{c_{1}}(\boldsymbol{u}, \boldsymbol{u}, \boldsymbol{v})-\mu c_{1}(\boldsymbol{v}, \boldsymbol{d}, \boldsymbol{w}) \\
+b(\boldsymbol{v}, p)=0 \quad \forall \boldsymbol{v} \in \boldsymbol{H}_{0}^{1}(\Omega), \\
\gamma(\boldsymbol{w}, \boldsymbol{e})+\mu\left\{d_{t}(\boldsymbol{d}, \boldsymbol{e})+c_{1}(\boldsymbol{u}, \boldsymbol{d}, \boldsymbol{e})+\gamma(\widetilde{\boldsymbol{f}}(\boldsymbol{d}), \boldsymbol{e})\right\}=0 \quad \forall \boldsymbol{e} \in \boldsymbol{L}^{2}(\Omega) .
\end{gathered}
$$

Finally, defining the spaces

$$
X=\boldsymbol{H}_{0}^{1}(\Omega) \times \boldsymbol{L}^{2}(\Omega), \quad Y=\boldsymbol{H}_{0}^{1}(\Omega) \times \boldsymbol{W}^{1,3}(\Omega), \quad M=L_{0}^{2}(\Omega) \times \boldsymbol{H}_{0}^{1}(\Omega),
$$

and the bilinear and trilinear forms $a: X \times X \rightarrow \mathbb{R}, c: Y \times X \times X \rightarrow \mathbb{R}$ through

$$
\begin{gathered}
a((\boldsymbol{u}, \boldsymbol{w}),(\boldsymbol{v}, \boldsymbol{e}))=a_{1}(\boldsymbol{u}, \boldsymbol{v})+\gamma(\boldsymbol{w}, \boldsymbol{e}) \quad \forall(\boldsymbol{u}, \boldsymbol{w}),(\boldsymbol{v}, \boldsymbol{e}) \in X, \\
\left\{\begin{array}{l}
c(\overline{\boldsymbol{u}}, \boldsymbol{d}),(\boldsymbol{u}, \boldsymbol{w}),(\boldsymbol{v}, \boldsymbol{e}))=\widetilde{c_{1}}(\overline{\boldsymbol{u}}, \boldsymbol{u}, \boldsymbol{v})+\mu\left\{c_{1}(\boldsymbol{u}, \boldsymbol{d}, \boldsymbol{e})-c_{1}(\boldsymbol{v}, \boldsymbol{d}, \boldsymbol{w})\right\}, \\
\forall(\overline{\boldsymbol{u}}, \boldsymbol{d}) \in Y, \quad \forall(\boldsymbol{u}, \boldsymbol{w}),(\boldsymbol{v}, \boldsymbol{e}) \in X,
\end{array}\right.
\end{gathered}
$$

we can express more compactly (4.3), (4.4) together with the last two lines of (4.2) and obtain the following problem: Find $\boldsymbol{u} \in L^{\infty}(\boldsymbol{H}) \cap L^{2}(\boldsymbol{V}), \boldsymbol{d} \in L^{\infty}\left(\boldsymbol{H}^{1}(\Omega)\right) \cap$ $L^{2}\left(\boldsymbol{H}^{3 / 2}(\Omega)\right), \boldsymbol{w} \in L^{2}\left(\boldsymbol{L}^{2}(\Omega)\right)$ and $p \in H^{-1}\left(0, T ; L_{0}^{2}(\Omega)\right)$ the solution of

$$
\left\{\begin{array}{c}
d_{t}((\boldsymbol{u}, \mu \boldsymbol{d}),(\boldsymbol{v}, \boldsymbol{e}))+a((\boldsymbol{u}, \boldsymbol{w}),(\boldsymbol{v}, \boldsymbol{e})) \\
+c((\boldsymbol{u}, \boldsymbol{d}),(\boldsymbol{u}, \boldsymbol{w}),(\boldsymbol{v}, \boldsymbol{e}))+b(\boldsymbol{v}, p)+\mu \gamma(\widetilde{\boldsymbol{f}}(\boldsymbol{d}), \boldsymbol{e})=0 \quad \forall(\boldsymbol{v}, \boldsymbol{e}) \in X \\
b(\boldsymbol{u}, q)+\mu(\nabla \widehat{\boldsymbol{d}}, \nabla \boldsymbol{g})-(\boldsymbol{w}, \boldsymbol{g})=0 \quad \forall(q, \boldsymbol{g}) \in M
\end{array}\right.
$$

with the initial conditions (3.6),

$$
\left.\boldsymbol{u}\right|_{t=0}=\left.\boldsymbol{u}_{0} \quad \boldsymbol{d}\right|_{t=0}=\boldsymbol{d}_{0}
$$

where $\widehat{\boldsymbol{d}}=\boldsymbol{d}-\tilde{\boldsymbol{d}}, \tilde{\boldsymbol{d}}$ solves (3.8):

$$
-\Delta \widetilde{\boldsymbol{d}}(t)=\mathbf{0} \text { in } \Omega,\left.\quad \tilde{\boldsymbol{d}}(t)\right|_{\partial \Omega}=\boldsymbol{l}(t) \text { on } \partial \Omega \text {, i.e., }(\nabla \widetilde{\boldsymbol{d}}, \nabla \boldsymbol{g})=0, \forall \boldsymbol{g} \in \boldsymbol{H}_{0}^{1}(\Omega),
$$

$\boldsymbol{l}$ is given in $H^{1}\left(0, T ; \boldsymbol{H}^{1}(\partial \Omega)\right)$ and $\left(\boldsymbol{u}_{0}, \boldsymbol{d}_{0}\right)$ in $\boldsymbol{H} \times \boldsymbol{H}^{1}(\Omega)$. The above considerations show that this problem is equivalent to (3.2)-(3.6).

The following properties of $a(\cdot, \cdot)$ and $b(\cdot, \cdot, \cdot)$ are easy to prove.

Lemma 4.1. The bilinear form a $(\cdot, \cdot): X \times X \rightarrow \mathbb{R}$ is continuous and coercive:

$$
\begin{gathered}
\forall(\boldsymbol{u}, \boldsymbol{w}),(\boldsymbol{v}, \boldsymbol{e}) \in X, \quad a((\boldsymbol{u}, \boldsymbol{w}),(\boldsymbol{v}, \boldsymbol{e})) \leq \max \{\nu, \gamma\}\|\boldsymbol{u}, \boldsymbol{w}\|_{X}\|\boldsymbol{v}, \boldsymbol{e}\|_{X}, \\
\forall(\boldsymbol{u}, \boldsymbol{w}) \in X, \quad a((\boldsymbol{u}, \boldsymbol{w}),(\boldsymbol{u}, \boldsymbol{w})) \geq \min \{\nu, \gamma\}\|\boldsymbol{u}, \boldsymbol{w}\|_{X}^{2} .
\end{gathered}
$$

The trilinear form $c(\cdot, \cdot, \cdot): Y \times X \times X \rightarrow \mathbb{R}$ is continuous:

$$
\begin{aligned}
& \forall(\overline{\boldsymbol{u}}, \boldsymbol{d}) \in Y, \forall(\boldsymbol{u}, \boldsymbol{w}),(\boldsymbol{v}, \boldsymbol{e}) \in X, \\
& \quad c((\overline{\boldsymbol{u}}, \boldsymbol{d}),(\boldsymbol{u}, \boldsymbol{w}),(\boldsymbol{v}, \boldsymbol{e})) \leq C\|\overline{\boldsymbol{u}}, \boldsymbol{d}\|_{\boldsymbol{L}^{3}(\Omega) \times \boldsymbol{W}^{1,3}(\Omega)}\|\boldsymbol{u}, \boldsymbol{w}\|_{X}\|\boldsymbol{v}, \boldsymbol{e}\|_{X},
\end{aligned}
$$


and verifies:

$$
\forall(\overline{\boldsymbol{u}}, \boldsymbol{d}) \in Y, \forall(\boldsymbol{u}, \boldsymbol{w}) \in X, \quad c((\overline{\boldsymbol{u}}, \boldsymbol{d}),(\boldsymbol{u}, \boldsymbol{w}),(\boldsymbol{u}, \boldsymbol{w}))=0 .
$$

\section{A FUlLy DISCRETE SCHEME}

Recall that $\Omega$ is a bounded, connected Lipschitz polyhedron in $\mathbb{R}^{3}$. Let $\mathcal{T}_{h}$ be a family of conforming triangulations of $\bar{\Omega}$, consisting of tetrahedra $T$ with diameter $h_{T}$ bounded by $h$, regular in the sense of Ciarlet [10]: there exists a constant $\sigma$, independent of $h$, such that

$$
\forall T \in \mathcal{T}_{h}, \frac{h_{T}}{\rho_{T}}:=\sigma_{T} \leq \sigma,
$$

where $\rho_{T}$ denotes the diameter of the ball inscribed in $T$. To simplify the discussion, we concentrate on finite element spaces of order one, but the following analysis carries over to elements of higher order. For an integer $k \geq 0$, let $\mathbb{P}_{k}$ denote the space of polynomials of total degree less than or equal to $k$. At each time step, we discretize the velocity $\boldsymbol{u}$ and pressure $p$ with a continuous stable approximation; more precisely, in each $T$ we take the mini-element $\left(\mathbb{P}_{1}+\mathbb{B}\right)^{3} \times \mathbb{P}_{1}$, where $\mathbb{B}$ is spanned by the bubble function

$$
b_{T}=\Pi_{i=1}^{4} \lambda_{i},
$$

and $\lambda_{i}$ are the barycentric coordinates of the vertices of $T$. It satisfies the inf-sup or LBB condition; cf. for instance [15] or [2]. Then, we discretize the direction vector $\boldsymbol{d}$ by a continuous approximation $\mathbb{P}_{1}$ in each $T$ and the auxiliary function $\boldsymbol{w}$ by discontinuous approximation $\mathbb{P}_{0}$ in each $T$. Thus, we introduce the finite element spaces:

$$
\begin{aligned}
& U_{h}=\left\{\boldsymbol{v}_{h} \in \mathcal{C}^{0}(\bar{\Omega})^{3} ; \forall T \in \mathcal{T}_{h},\left.\boldsymbol{v}_{h}\right|_{T} \in\left(\mathbb{P}_{1}+\mathbb{B}\right)^{3}\right\} \\
& U_{0 h}=U_{h} \cap \boldsymbol{H}_{0}^{1}(\Omega) \quad(\text { velocity }) \\
& P_{h}=\left\{q_{h} \in \mathcal{C}^{0}(\bar{\Omega}) ; \forall T \in \mathcal{T}_{h},\left.q_{h}\right|_{T} \in \mathbb{P}_{1}\right\} \cap L_{0}^{2}(\Omega) \quad \text { (pressure) }, \\
& W_{h}=\left\{\boldsymbol{w}_{h} \in L^{2}(\Omega)^{3} ; \forall T \in \mathcal{T}_{h},\left.\boldsymbol{w}_{h}\right|_{T} \in \mathbb{P}_{0}^{3}\right\} \quad \text { (auxiliary function), } \\
& D_{h}=\left\{\boldsymbol{g}_{h} \in \mathcal{C}^{0}(\bar{\Omega})^{3} ; \forall T \in \mathcal{T}_{h},\left.\boldsymbol{g}_{h}\right|_{T} \in \mathbb{P}_{1}^{3}\right\} \\
& D_{0 h}=D_{h} \cap \boldsymbol{H}_{0}^{1}(\Omega) \quad(\text { direction vector }), \\
& V_{h}=\left\{\boldsymbol{v}_{h} \in U_{0 h} ; \forall q_{h} \in P_{h}, b\left(\boldsymbol{v}_{h}, q_{h}\right)=0\right\}
\end{aligned}
$$

(discrete divergence-free velocity),

and we set $X_{h}=U_{0 h} \times W_{h}$ and $M_{h}=P_{h} \times D_{0 h}$.

As far as discretization in time is concerned, we choose an integer $M \geq 2$ and define the time step and subdivision points:

$$
k=\frac{T}{M}, \quad t_{n}=n k, 0 \leq n \leq M .
$$

For a sequence $\left(v^{n}\right)_{n}$, we denote the (backward) divided difference in time by

$$
\delta_{t} v^{n}=\frac{1}{k}\left(v^{n}-v^{n-1}\right)
$$

Next, we define a discrete lifting of the boundary data $\boldsymbol{l}$. As $\Omega$ is a polyhedron, the trace of $\mathcal{T}_{h}$ on $\partial \Omega$ induces a conforming triangulation, $\partial \mathcal{T}_{h}$ of $\partial \Omega$. Let $S Z_{h}$ denote the following Scott \& Zhang [34] regularization operator on $D_{h}$. With each 
node $\boldsymbol{a}$ of $\mathcal{T}_{h}$, we associate once and for all a face $e_{\boldsymbol{a}}$ of $\mathcal{T}_{h}$ with vertex $\boldsymbol{a}$, with the restriction that $e_{\boldsymbol{a}}$ be contained in $\partial \Omega$ when $\boldsymbol{a}$ is a boundary node. Then, for any function $v \in W^{1,1}(\Omega)$, and all vertices $\boldsymbol{a}$ of $\mathcal{T}_{h}$, we define

$$
S Z_{h} v(\boldsymbol{a})=\frac{1}{\left|e_{\boldsymbol{a}}\right|} \int_{e_{\boldsymbol{a}}} v(s) d s
$$

and we interpolate these values with $\mathbb{P}_{1}$ functions in each $T$. The operator $S Z_{h}$ is stable in $W^{1,1}(\Omega)$ and has optimal approximation properties. The trace of $S Z_{h} v$ on $\partial \Omega$ depends only on the trace of $v$ on $\partial \Omega$ and vanishes if $v$ vanishes on $\partial \Omega$. Thus it defines an interpolant of $\left.v\right|_{\partial \Omega}$ on $\partial \mathcal{T}_{h}$ that we denote by $S Z_{h} v$. Furthermore, it follows easily from (5.8) that the constraint $|\boldsymbol{d}| \leq 1$ implies $\left|S Z_{h} \boldsymbol{d}\right| \leq 1$. Now, recall that $\boldsymbol{l}$ is continuous in time and belongs to $\boldsymbol{H}^{1}(\partial \Omega)$; therefore at each time $t_{n}$ we define the discrete boundary values by $\boldsymbol{l}_{h}^{n}=S Z_{h}\left(\boldsymbol{l}\left(t_{n}\right)\right)$. For analyzing the numerical scheme, it is convenient to introduce the solution $\widetilde{\boldsymbol{d}}_{h}^{n} \in D_{h}$ of the discrete problem

$$
\left.\widetilde{\boldsymbol{d}}_{h}^{n}\right|_{\partial \Omega}=\boldsymbol{l}_{h}^{n} \quad \text { and } \quad \forall \boldsymbol{g}_{h} \in D_{0 h},\left(\nabla \widetilde{\boldsymbol{d}}_{h}^{n}, \nabla \boldsymbol{g}_{h}\right)=0
$$

By using the stability of $\left.S Z_{h}\right|_{\partial \Omega}$ in $H^{1 / 2}(\partial \Omega)$, one has

$$
\left\|\widetilde{\boldsymbol{d}}_{h}^{n}\right\|_{\boldsymbol{H}^{1}(\Omega)} \leq C\left\|\boldsymbol{l}_{h}^{n}\right\|_{\boldsymbol{H}^{1 / 2}(\partial \Omega)}=\left\|S Z_{h} \boldsymbol{l}\left(t_{n}\right)\right\|_{\boldsymbol{H}^{1 / 2}(\partial \Omega)} \leq C\left\|\boldsymbol{l}\left(t_{n}\right)\right\|_{\boldsymbol{H}^{1 / 2}(\partial \Omega)} .
$$

As an initial step, we take for $\boldsymbol{u}_{h}^{0}$ a suitable approximation of $\boldsymbol{u}_{0}$ in $U_{0 h}$, stable in $\boldsymbol{L}^{2}(\Omega)$, we take $\boldsymbol{d}_{h}^{0}=S Z_{h} \boldsymbol{d}_{0}$ and we set $\widehat{\boldsymbol{d}}_{h}^{0}=\boldsymbol{d}_{h}^{0}-\widetilde{\boldsymbol{d}}_{h}^{0}$. In particular, $\boldsymbol{d}_{h}^{0}$ and $\widehat{\boldsymbol{d}}_{h}^{0}$ are approximations of $\boldsymbol{d}_{0}$ and $\widehat{\boldsymbol{d}}_{0}$ in $D_{h}$ and $D_{0 h}$, respectively, both stable in $\boldsymbol{H}^{1}(\Omega)$.

Then the fully discrete scheme proceeds as follows:

Step $n$ in time $(n \geq 1)$. Knowing $\left(\boldsymbol{u}_{h}^{n-1}, \widehat{\boldsymbol{d}}_{h}^{n-1}\right) \in U_{0 h} \times D_{0 h}\left(\right.$ with $\boldsymbol{d}_{h}^{n-1}=\widehat{\boldsymbol{d}}_{h}^{n-1}+$ $\widetilde{\boldsymbol{d}}_{h}^{n-1}$ ), find $\left(\boldsymbol{u}_{h}^{n}, \boldsymbol{w}_{h}^{n}\right) \in X_{h}$ and $\left(p_{h}^{n}, \widehat{\boldsymbol{d}}_{h}^{n}\right) \in M_{h}$ (with $\left.\boldsymbol{d}_{h}^{n}=\widehat{\boldsymbol{d}}_{h}^{n}+\widetilde{\boldsymbol{d}}_{h}^{n}\right)$ solution of:

$$
\begin{aligned}
& \left(\delta_{t}\left(\boldsymbol{u}_{h}^{n}, \mu \widehat{\boldsymbol{d}}_{h}^{n}\right),(\boldsymbol{v}, \boldsymbol{e})\right)+a\left(\left(\boldsymbol{u}_{h}^{n}, \boldsymbol{w}_{h}^{n}\right),(\boldsymbol{v}, \boldsymbol{e})\right)+b\left(\boldsymbol{v}, p_{h}^{n}\right) \\
& +c\left(\left(\boldsymbol{u}_{h}^{n-1}, \boldsymbol{d}_{h}^{n-1}\right),\left(\boldsymbol{u}_{h}^{n}, \boldsymbol{w}_{h}^{n}\right),(\boldsymbol{v}, \boldsymbol{e})\right) \\
& =-\mu\left(\gamma \widetilde{\boldsymbol{f}}\left(\boldsymbol{d}_{h}^{n-1}\right)+\delta_{t} \widetilde{\boldsymbol{d}}_{h}^{n}, \boldsymbol{e}\right) \quad \forall(\boldsymbol{v}, \boldsymbol{e}) \in X_{h}, \\
& b\left(\boldsymbol{u}_{h}^{n}, q\right)+\mu\left(\nabla \widehat{\boldsymbol{d}}_{h}^{n}, \nabla \boldsymbol{g}\right)-\left(\boldsymbol{w}_{h}^{n}, \boldsymbol{g}\right)=0 \quad \forall(q, \boldsymbol{g}) \in M_{h} .
\end{aligned}
$$

It is easy to check that (5.10), (5.11) is a square linear system in finite dimension; therefore, uniqueness of its solution implies existence. Uniqueness of $\boldsymbol{u}_{h}^{n}, \boldsymbol{w}_{h}^{n}$ and $\widehat{\boldsymbol{d}}_{h}^{n}$ follows from the energy estimates (5.13) in the next subsection; then uniqueness of $p_{h}^{n}$ is a consequence of the inf-sup condition (see [3], [7], or [15]).

Remark 5.1. Of course, the particular lifting $\widetilde{\boldsymbol{d}}_{h}^{n}$ satisfying (5.9) is only a matter of theoretical convenience and (5.10), (5.11) is equivalent to: Knowing $\left(\boldsymbol{u}_{h}^{n-1}, \boldsymbol{d}_{h}^{n-1}\right) \in$ 
$U_{0 h} \times D_{h}$, find $\left(\boldsymbol{u}_{h}^{n}, \boldsymbol{w}_{h}^{n}\right)$ and $\left(p_{h}^{n}, \boldsymbol{d}_{h}^{n}\right)$ with $\left.\boldsymbol{d}_{h}^{n}\right|_{\partial \Omega}=\boldsymbol{l}_{h}^{n}$, the solution of:

$$
\begin{aligned}
& \left(\delta_{t}\left(\boldsymbol{u}_{h}^{n}, \mu \boldsymbol{d}_{h}^{n}\right),(\boldsymbol{v}, \boldsymbol{e})\right)+a\left(\left(\boldsymbol{u}_{h}^{n}, \boldsymbol{w}_{h}^{n}\right),(\boldsymbol{v}, \boldsymbol{e})\right)+b\left(\boldsymbol{v}, p_{h}^{n}\right) \\
& \quad+c\left(\left(\boldsymbol{u}_{h}^{n-1}, \boldsymbol{d}_{h}^{n-1}\right),\left(\boldsymbol{u}_{h}^{n}, \boldsymbol{w}_{h}^{n}\right),(\boldsymbol{v}, \boldsymbol{e})\right) \\
& \quad=-\mu\left(\gamma \widetilde{\boldsymbol{f}}\left(\boldsymbol{d}_{h}^{n-1}\right), \boldsymbol{e}\right) \quad \forall(\boldsymbol{v}, \boldsymbol{e}) \in X_{h}, \\
& b\left(\boldsymbol{u}_{h}^{n}, q\right)+\mu\left(\nabla \boldsymbol{d}_{h}^{n}, \nabla \boldsymbol{g}\right)-\left(\boldsymbol{w}_{h}^{n}, \boldsymbol{g}\right)=0 \quad \forall(q, \boldsymbol{g}) \in M_{h} .
\end{aligned}
$$

Moreover, in practical situations, we can assume that $\boldsymbol{l}$ is continuous in space (e.g. a little better than $\boldsymbol{H}^{1}(\partial \Omega)$ when $N=3$ ). Then for implementing (5.12), we approximate $\boldsymbol{l}$ by interpolating its pointwise values on $\partial \mathcal{T}_{h}$.

Remark 5.2. Owing to the degree of the polynomials, if the inner dihedral angles of each $T$ in $\mathcal{T}_{h}$ is not larger than $\pi / 2$, then $\widetilde{\boldsymbol{d}}_{h}^{n}$ defined by (5.9) satisfies the maximum principle (cf. Ciarlet [10]) and hence

$$
\forall \boldsymbol{x} \in \Omega,\left|\widetilde{\boldsymbol{d}}_{h}^{n}(\boldsymbol{x})\right| \leq 1
$$

5.1. Energy estimates. In the sequel, $C$ denotes different constants, always independent of $k$ and $h$, but possibly depending exponentially on $1 / \varepsilon^{2}$.

The following stability result is obtained by using standard techniques in the Navier-Stokes framework: by choosing $(\boldsymbol{v}, \boldsymbol{e})=\left(\boldsymbol{u}_{h}^{n}, \boldsymbol{w}_{h}^{n}\right) \in X_{h}$ as test functions in (5.10) and $(q, \boldsymbol{g})=\left(p_{h}^{n}, \mu \delta_{t} \widehat{\boldsymbol{d}}_{h}^{n}\right) \in M_{h}$ in (5.11), taking into account that

$$
k \sum_{n \geq 1}\left\|\delta_{t} \widetilde{\boldsymbol{d}}_{h}^{n}\right\|_{\boldsymbol{L}^{2}(\Omega)}^{2} \leq C \int_{0}^{T}\left\|\partial_{t} \boldsymbol{l}(t)\right\|_{\boldsymbol{H}^{1 / 2}(\partial \Omega)}^{2} d t \leq C,
$$

and using the coercivity of $a(\cdot, \cdot)$ and the antisymmetry of $c(\cdot, \cdot, \cdot)$ given in (4.8), i.e.,

$$
c\left(\left(\boldsymbol{u}_{h}^{n-1}, \boldsymbol{d}_{h}^{n-1}\right),\left(\boldsymbol{u}_{h}^{n}, \boldsymbol{w}_{h}^{n}\right),\left(\boldsymbol{u}_{h}^{n}, \boldsymbol{w}_{h}^{n}\right)\right)=0 .
$$

Lemma 5.3. There exists $C=C\left(\Omega, \nu, \gamma, \lambda,\left\|\boldsymbol{u}_{0}, \boldsymbol{d}_{0}\right\|_{\boldsymbol{L}^{2}(\Omega) \times \boldsymbol{H}^{1}(\Omega)}, \boldsymbol{l}, \varepsilon\right)>0$ such that

$$
\begin{aligned}
& \sup _{0 \leq n \leq M}\left\|\boldsymbol{u}_{h}^{n}, \widehat{\boldsymbol{d}}_{h}^{n}\right\|_{\boldsymbol{L}^{2}(\Omega) \times \boldsymbol{H}^{1}(\Omega)} \leq C, \quad k \sum_{n=1}^{M}\left\|\boldsymbol{u}_{h}^{n}, \boldsymbol{w}_{h}^{n}\right\|_{\boldsymbol{H}^{1}(\Omega) \times \boldsymbol{L}^{2}(\Omega)}^{2} \leq C, \\
& \sum_{n=1}^{M}\left\|\boldsymbol{u}_{h}^{n}-\boldsymbol{u}_{h}^{n-1}, \widehat{\boldsymbol{d}}_{h}^{n}-\widehat{\boldsymbol{d}}_{h}^{n-1}\right\|_{\boldsymbol{L}^{2}(\Omega) \times \boldsymbol{H}^{1}(\Omega)}^{2} \leq C .
\end{aligned}
$$

As $\left\|\widetilde{\boldsymbol{d}}_{h}^{n}\right\|_{\boldsymbol{H}^{1}(\Omega)} \leq C\left\|\boldsymbol{l}\left(t_{n}\right)\right\|_{\boldsymbol{H}^{1 / 2}(\partial \Omega)}$, the assumptions on $\boldsymbol{l}$ imply that

$$
\sup _{0 \leq n \leq M}\left\|\widetilde{\boldsymbol{d}}_{h}^{n}\right\|_{\boldsymbol{H}^{1}(\Omega)} \leq C
$$

therefore, the first part of (5.13) yields

$$
\sup _{0 \leq n \leq M}\left\|\boldsymbol{d}_{h}^{n}\right\|_{\boldsymbol{H}^{1}(\Omega)} \leq C .
$$

Remark 5.4. When applying Gronwall's Lemma in the proof of Lemma 5.3, the term $\mu \gamma \tilde{\boldsymbol{f}}\left(\boldsymbol{d}_{h}^{n-1}\right)$ in the right-hand side of (5.10) is responsible for the exponential dependence of $C$ on $1 / \varepsilon^{2}$. 
5.2. $W^{1,3}$ a priori estimates in space for the director field. From now on, in order to apply some inverse inequalities, we assume that the triangulation of $\Omega$ is quasi-uniform: There exists a constant $\tau$, independent of $h$, such that

$$
\forall T \in \mathcal{T}_{h}, \tau h \leq h_{T} \leq \sigma \rho_{T} .
$$

The following crucial result enables us to bypass a $\mathcal{C}^{1}$ discretization of $\boldsymbol{d}$.

Lemma 5.5. There exists a constant $C>0$ (independent of $h$ and $k$ ), such that, for each $n \geq 1$, given $\boldsymbol{w}_{h}^{n}$, the solution $\widehat{\boldsymbol{d}}_{h}^{n}$ of (5.11) satisfies:

$$
\left\|\widehat{\boldsymbol{d}}_{h}^{n}\right\|_{\boldsymbol{W}^{1, r}(\Omega)} \leq C\left\|\boldsymbol{w}_{h}^{n}\right\|_{\boldsymbol{L}^{s}(\Omega)},
$$

where $r=3$ and $s=3 / 2$ if $N=3$, and $r=4$ and $s=4 / 3$ if $N=2$. In particular, for each $n \geq 1$,

$$
\left\|\widehat{\boldsymbol{d}}_{h}^{n}\right\|_{\boldsymbol{W}^{1,3}(\Omega)} \leq C\left\|\boldsymbol{w}_{h}^{n}\right\|_{\boldsymbol{L}^{2}(\Omega)} .
$$

Proof. We sketch the proof for the reader's convenience; it is a standard application of Sobolev imbeddings, elliptic $L^{p}$ regularity, inverse inequalities and approximation properties of $S Z_{h}$ (cf. for instance [15]). Let $\boldsymbol{w}_{h}^{n} \in W_{h}$ and let $\widehat{\boldsymbol{d}}(h) \in \boldsymbol{H}^{1}(\Omega)$ solve the problem:

$$
-\mu \Delta \widehat{\boldsymbol{d}}(h)=\boldsymbol{w}_{h}^{n} \text { in } \Omega, \quad \widehat{\boldsymbol{d}}(h)=0 \text { on } \partial \Omega .
$$

According to Theorems 2.1 and 2.3, we have

- If $N=2$, then $\widehat{\boldsymbol{d}}(h) \in \boldsymbol{W}^{2,4 / 3}(\Omega)$ and $\|\widehat{\boldsymbol{d}}(h)\|_{\boldsymbol{W}^{2,4 / 3}(\Omega)} \leq C\left\|\boldsymbol{w}_{h}^{n}\right\|_{\boldsymbol{L}^{4 / 3}(\Omega)}$.

- If $N=3$, then $\widehat{\boldsymbol{d}}(h) \in \boldsymbol{H}^{3 / 2}(\Omega)$ and $\|\widehat{\boldsymbol{d}}(h)\|_{\boldsymbol{H}^{3 / 2}(\Omega)} \leq C\left\|\boldsymbol{w}_{h}^{n}\right\|_{\boldsymbol{L}^{3 / 2}(\Omega)}$.

When $N=2$, Sobolev imbedding $W^{2,4 / 3}(\Omega) \hookrightarrow W^{1,4}(\Omega)$ yields $\|\widehat{\boldsymbol{d}}(h)\|_{W^{1,4}(\Omega)} \leq$ $C\left\|\boldsymbol{w}_{h}^{n}\right\|_{L^{4 / 3}(\Omega)}$, and when $N=3$, Sobolev imbedding $H^{3 / 2}(\Omega) \hookrightarrow W^{1,3}(\Omega)$ gives

$$
\|\widehat{\boldsymbol{d}}(h)\|_{\boldsymbol{W}^{1,3}(\Omega)} \leq C\left\|\boldsymbol{w}_{h}^{n}\right\|_{\boldsymbol{L}^{3 / 2}(\Omega)} .
$$

Let us write the remainder of the proof for $N=3$, the proof being similar and giving a sharper result when $N=2$. We split $\nabla \widehat{\boldsymbol{d}}_{h}^{n}$ as follows:

$$
\begin{aligned}
\left\|\nabla \widehat{\boldsymbol{d}}_{h}^{n}\right\|_{L^{3}(\Omega)} \leq & \left\|\nabla\left(\widehat{\boldsymbol{d}}_{h}^{n}-S Z_{h} \widehat{\boldsymbol{d}}(h)\right)\right\|_{L^{3}(\Omega)}+\left\|\nabla\left(S Z_{h} \widehat{\boldsymbol{d}}(h)-\widehat{\boldsymbol{d}}(h)\right)\right\|_{L^{3}(\Omega)} \\
& +\|\nabla \widehat{\boldsymbol{d}}(h)\|_{L^{3}(\Omega)} .
\end{aligned}
$$

The last term in the above right-hand side is bounded by (5.20) and a bound for the second one follows easily from the stability of $S Z_{h}$ and (5.20):

$$
\left\|S Z_{h} \widehat{\boldsymbol{d}}(h)-\widehat{\boldsymbol{d}}(h)\right\|_{W^{1,3}(\Omega)} \leq C\|\widehat{\boldsymbol{d}}(h)\|_{W^{1,3}(\Omega)} \leq C\left\|\boldsymbol{w}_{h}^{n}\right\|_{\boldsymbol{L}^{3 / 2}(\Omega)} .
$$

Therefore, it suffices to estimate the first term. For this, setting (5.19) into variational form and comparing with (5.11) with $q=0$, we obtain the orthogonality relation

$$
\left(\nabla\left(\widehat{\boldsymbol{d}}_{h}^{n}-\widehat{\boldsymbol{d}}(h)\right), \nabla \boldsymbol{g}\right)=0 \quad \forall \boldsymbol{g} \in D_{0 h}
$$


Consequently, taking $\boldsymbol{g}=\widehat{\boldsymbol{d}}_{h}^{n}-S Z_{h} \widehat{\boldsymbol{d}}(h) \in D_{0 h}$, using the approximation properties of $S Z_{h}$ and (5.20), we derive

$$
\begin{aligned}
\left\|\nabla\left(\widehat{\boldsymbol{d}}_{h}^{n}-S Z_{h} \widehat{\boldsymbol{d}}(h)\right)\right\|_{\boldsymbol{L}^{2}(\Omega)} & \leq\left\|\nabla\left(\widehat{\boldsymbol{d}}(h)-S Z_{h} \widehat{\boldsymbol{d}}(h)\right)\right\|_{\boldsymbol{L}^{2}(\Omega)} \\
& \leq C h^{1 / 2}\|\widehat{\boldsymbol{d}}(h)\|_{\boldsymbol{H}^{3 / 2}(\Omega)} \\
& \leq C h^{1 / 2}\left\|\boldsymbol{w}_{h}^{n}\right\|_{\boldsymbol{L}^{3 / 2}(\Omega)} .
\end{aligned}
$$

Then the inverse inequality (that holds on any finite-element space defined on a quasi-uniform triangulation in three dimensions)

$$
\left\|v_{h}\right\|_{L^{3}(\Omega)} \leq C h^{-1 / 2}\left\|v_{h}\right\|_{L^{2}(\Omega)},
$$

yields

$$
\left\|\nabla\left(\widehat{\boldsymbol{d}}_{h}^{n}-S Z_{h} \widehat{\boldsymbol{d}}(h)\right)\right\|_{\boldsymbol{L}^{3}(\Omega)} \leq C\left\|\boldsymbol{w}_{h}^{n}\right\|_{\boldsymbol{L}^{3 / 2}(\Omega)},
$$

and (5.17) follows from these inequalities.

Remark 5.6. When $\Omega$ is convex, as (5.21) means that $\widehat{\boldsymbol{d}}_{h}^{n}$ is the finite-element elliptic projection of $\widehat{\boldsymbol{d}}(h)$, the sharp results of [33] and [6] give immediately

$$
\left\|\nabla \widehat{\boldsymbol{d}}_{h}^{n}\right\|_{\boldsymbol{L}^{3}(\Omega)} \leq\|\nabla \widehat{\boldsymbol{d}}(h)\|_{\boldsymbol{L}^{3}(\Omega)} .
$$

Also, in this case, $\widehat{\boldsymbol{d}}(h)$ belongs to $\boldsymbol{H}^{2}(\Omega)$ and the above argument permits us to somewhat relax the quasi-uniformity of the triangulation. But we prefer to avoid this convexity assumption.

Note that (5.13) and (5.18) imply

$$
k \sum_{n \geq 1}\left\|\widehat{\boldsymbol{d}}_{h}^{n}\right\|_{\boldsymbol{W}^{1,3}(\Omega)}^{2} \leq C .
$$

Furthermore, the proof of Lemma 5.5 also shows that

$$
\left\|\tilde{\boldsymbol{d}}_{h}^{n}\right\|_{\boldsymbol{W}^{1,3}(\Omega)} \leq C\left\|\boldsymbol{l}\left(t_{n}\right)\right\|_{\boldsymbol{H}^{1}(\partial \Omega)} .
$$

Indeed, as $\boldsymbol{l}\left(t_{n}\right)$ belongs to $\boldsymbol{H}^{1}(\partial \Omega)$, so does $\boldsymbol{l}_{h}^{n}$ and by virtue of Theorem 2.3, the solution $\widetilde{\boldsymbol{d}}(h) \in \boldsymbol{H}^{1}(\Omega)$ of

$$
-\Delta \widetilde{\boldsymbol{d}}(h)=\mathbf{0} \quad \text { in } \Omega, \quad \tilde{\boldsymbol{d}}(h)=\boldsymbol{l}_{h}^{n} \quad \text { on } \partial \Omega,
$$

belongs to $\boldsymbol{H}^{3 / 2}(\Omega)$ and $\|\widetilde{\boldsymbol{d}}(h)\|_{\boldsymbol{H}^{3 / 2}(\Omega)} \leq C\left\|\boldsymbol{l}\left(t_{n}\right)\right\|_{\boldsymbol{H}^{1 / 2}(\partial \Omega)}$. Then the proof of (5.25) proceeds as in Lemma 5.5. Hence

$$
k \sum_{n \geq 1}\left\|\widetilde{\boldsymbol{d}}_{h}^{n}\right\|_{\boldsymbol{W}^{1,3}(\Omega)}^{2} \leq C,
$$

and therefore

$$
k \sum_{n \geq 1}\left\|\boldsymbol{d}_{h}^{n}\right\|_{\boldsymbol{W}^{1,3}(\Omega)}^{2} \leq C .
$$


5.3. A priori fractional estimates in time for the discrete velocity and director fields. To prove compactness in time of $\boldsymbol{u}_{h}^{n}$ and $\boldsymbol{d}_{h}^{n}$, let us sharpen (5.14) as follows.

Proposition 5.7. There exists a constant $C>0$, independent of $h$ and $k$ but depending on $\varepsilon$, such that for all integers $\ell$ with $1 \leq \ell \leq M-1$,

$$
I_{\ell}:=k \sum_{m=1}^{M-\ell}\left\|\boldsymbol{u}_{h}^{m+\ell}-\boldsymbol{u}_{h}^{m}, \mu\left(\widehat{\boldsymbol{d}}_{h}^{m+\ell}-\widehat{\boldsymbol{d}}_{h}^{m}\right)\right\|_{L^{2}(\Omega) \times \boldsymbol{H}^{1}(\Omega)}^{2} \leq C(\ell k)^{1 / 2} .
$$

Proof. Let us sum (5.10) over $n$ from $n=m+1$ to $m+\ell$ and take $(\boldsymbol{v}, \boldsymbol{e})=$ $\left(\boldsymbol{u}_{h}^{m+\ell}-\boldsymbol{u}_{h}^{m}, \boldsymbol{w}_{h}^{m+\ell}-\boldsymbol{w}_{h}^{m}\right)$ as test functions. Owing to (5.11), we have

$$
\mu\left(\nabla\left(\widehat{\boldsymbol{d}}_{h}^{m+\ell}-\widehat{\boldsymbol{d}}_{h}^{m}\right), \nabla \boldsymbol{g}\right)=\left(\boldsymbol{w}_{h}^{m+\ell}-\boldsymbol{w}_{h}^{m}, \boldsymbol{g}\right), \quad b\left(\boldsymbol{u}_{h}^{m+\ell}-\boldsymbol{u}_{h}^{m}, q\right)=0 .
$$

This gives

$$
\left(\mu\left(\widehat{\boldsymbol{d}}_{h}^{m+\ell}-\widehat{\boldsymbol{d}}_{h}^{m}\right), \boldsymbol{w}_{h}^{m+\ell}-\boldsymbol{w}_{h}^{m}\right)=\left\|\mu \nabla\left(\widehat{\boldsymbol{d}}_{h}^{m+\ell}-\widehat{\boldsymbol{d}}_{h}^{m}\right)\right\|_{\boldsymbol{L}^{2}(\Omega)}^{2}
$$

and

$$
\sum_{n=m+1}^{m+\ell} b\left(\boldsymbol{u}_{h}^{m+\ell}-\boldsymbol{u}_{h}^{m}, p_{h}^{n}\right)=0 .
$$

Therefore,

$$
\begin{aligned}
& \left\|\boldsymbol{u}_{h}^{m+\ell}-\boldsymbol{u}_{h}^{m}, \mu\left(\widehat{\boldsymbol{d}}_{h}^{m+\ell}-\widehat{\boldsymbol{d}}_{h}^{m}\right)\right\|_{\boldsymbol{L}^{2}(\Omega) \times \boldsymbol{H}^{1}(\Omega)}^{2} \\
= & -k \sum_{n=m+1}^{m+\ell} a\left(\left(\boldsymbol{u}_{h}^{n}, \boldsymbol{w}_{h}^{n}\right),\left(\boldsymbol{u}_{h}^{m+\ell}-\boldsymbol{u}_{h}^{m}, \boldsymbol{w}_{h}^{m+\ell}-\boldsymbol{w}_{h}^{m}\right)\right) \\
& -k \sum_{n=m+1}^{m+\ell} c\left(\left(\boldsymbol{u}_{h}^{n-1}, \boldsymbol{d}_{h}^{n-1}\right),\left(\boldsymbol{u}_{h}^{n}, \boldsymbol{w}_{h}^{n}\right),\left(\boldsymbol{u}_{h}^{m+\ell}-\boldsymbol{u}_{h}^{m}, \boldsymbol{w}_{h}^{m+\ell}-\boldsymbol{w}_{h}^{m}\right)\right) \\
& -k \mu \sum_{n=m+1}^{m+\ell}\left(\gamma \widetilde{\boldsymbol{f}}\left(\boldsymbol{d}_{h}^{n-1}\right)+\delta_{t} \widetilde{\boldsymbol{d}}_{h}^{n}, \boldsymbol{w}_{h}^{m+\ell}-\boldsymbol{w}_{h}^{m}\right) .
\end{aligned}
$$

Let us multiply the above expression by $k$, sum it over $m$ from $m=1$ to $m=M-\ell$ and invert the order of summation; then $n$ runs from 2 to $M$ and $m$ runs from $\max (1, n-\ell)$ to $\min (n-1, M-\ell)$. The contribution to $I_{\ell}$ of the term involving $a_{1}$ has the bound:

$$
\begin{aligned}
& k^{2}\left|\sum_{m=1}^{M-\ell} \sum_{n=m+1}^{m+\ell} \nu\left(\nabla \boldsymbol{u}_{h}^{n}, \nabla\left(\boldsymbol{u}_{h}^{m+\ell}-\boldsymbol{u}_{h}^{m}\right)\right)\right| \\
& \leq k^{2} \nu \sum_{n=2}^{M}\left\|\nabla \boldsymbol{u}_{h}^{n}\right\|_{L^{2}(\Omega)} \sum_{m=\max (1, n-\ell)}^{\min (n-1, M-\ell)}\left\|\nabla\left(\boldsymbol{u}_{h}^{m+\ell}-\boldsymbol{u}_{h}^{m}\right)\right\|_{\boldsymbol{L}^{2}(\Omega)} .
\end{aligned}
$$

But

$$
\min (n-1, M-\ell)-\max (1, n-\ell) \leq \ell-1 .
$$


Therefore, a Cauchy-Schwarz inequality applied to both sums yields

$$
\begin{gathered}
k^{2}\left|\sum_{m=1}^{M-\ell} \sum_{n=m+1}^{m+\ell} \nu\left(\nabla \boldsymbol{u}_{h}^{n}, \nabla\left(\boldsymbol{u}_{h}^{m+\ell}-\boldsymbol{u}_{h}^{m}\right)\right)\right| \leq \nu(k(\ell-1))^{1 / 2}(k(M-1))^{1 / 2} \\
\times\left(\sum_{n=2}^{M} k\left\|\nabla \boldsymbol{u}_{h}^{n}\right\|_{\boldsymbol{L}^{2}(\Omega)}^{2}\right)^{1 / 2}\left(\sum_{m=1}^{M-\ell} k\left\|\nabla\left(\boldsymbol{u}_{h}^{m+\ell}-\boldsymbol{u}_{h}^{m}\right)\right\|_{\boldsymbol{L}^{2}(\Omega)}^{2}\right)^{1 / 2} .
\end{gathered}
$$

In view of the second part of (5.13), both sums in this right-hand side are bounded by a constant $C$, independent of $h$ and $k$. Thus

$$
k^{2}\left|\sum_{m=1}^{M-\ell} \sum_{n=m+1}^{m+\ell} \nu\left(\nabla \boldsymbol{u}_{h}^{n}, \nabla\left(\boldsymbol{u}_{h}^{m+\ell}-\boldsymbol{u}_{h}^{m}\right)\right)\right| \leq C \nu(k \ell)^{1 / 2} T^{1 / 2}
$$

By replacing all occurrences of $\nabla \boldsymbol{u}_{h}^{i}$ by $\boldsymbol{w}_{h}^{i}$, the term involving $a_{2}$ has a similar bound with another constant $C$ :

$$
k^{2}\left|\sum_{m=1}^{M-\ell} \sum_{n=m+1}^{m+\ell} \gamma\left(\boldsymbol{w}_{h}^{n}, \boldsymbol{w}_{h}^{m+\ell}-\boldsymbol{w}_{h}^{m}\right)\right| \leq C \gamma(k \ell)^{1 / 2} T^{1 / 2} .
$$

As far as the non-linear terms are concerned, consider the term $\left(\left(\nabla \boldsymbol{d}_{h}^{n-1}\right)^{t} \boldsymbol{w}_{h}^{n}\right.$, $\left.\boldsymbol{u}_{h}^{m+\ell}-\boldsymbol{u}_{h}^{m}\right)$. By the above argument and Sobolev imbeddings, we obtain

$$
\begin{aligned}
\mu k^{2} \mid & \sum_{m=1}^{M-\ell} \sum_{n=m+1}^{m+\ell}\left(\left(\nabla \boldsymbol{d}_{h}^{n-1}\right)^{t} \boldsymbol{w}_{h}^{n}, \boldsymbol{u}_{h}^{m+\ell}-\boldsymbol{u}_{h}^{m}\right) \mid \leq C \mu(k(\ell-1))^{1 / 2} \\
& \times\left(\sum_{n=2}^{M} k\left\|\boldsymbol{w}_{h}^{n}\right\|_{\boldsymbol{L}^{2}(\Omega)}^{2}\right)^{1 / 2}\left(\sum_{n=1}^{M-1} k\left\|\nabla \boldsymbol{d}_{h}^{n}\right\|_{\boldsymbol{L}^{3}(\Omega)}^{2}\right)^{1 / 2} \\
& \times\left(\sum_{m=1}^{M-\ell} k\left\|\nabla\left(\boldsymbol{u}_{h}^{m+\ell}-\boldsymbol{u}_{h}^{m}\right)\right\|_{\boldsymbol{L}^{2}(\Omega)}^{2}\right)^{1 / 2} \leq C \mu(k \ell)^{1 / 2}
\end{aligned}
$$

owing to the second part of (5.13) and (5.26).

In view of (3.17), the term involving $\widetilde{\boldsymbol{f}}$ has the bound

$$
\begin{gathered}
\mu k^{2}\left|\sum_{m=1}^{M-\ell} \sum_{n=m+1}^{m+\ell} \gamma\left(\tilde{\boldsymbol{f}}\left(\boldsymbol{d}_{h}^{n-1}\right), \boldsymbol{w}_{h}^{m+\ell}-\boldsymbol{w}_{h}^{m}\right)\right| \\
\leq \gamma \frac{\mu}{\varepsilon^{2}}|\Omega|^{1 / 2} T(k(\ell-1))^{1 / 2}\left(\sum_{m=1}^{M-\ell} k\left\|\boldsymbol{w}_{h}^{m+\ell}-\boldsymbol{w}_{h}^{m}\right\|_{\boldsymbol{L}^{2}(\Omega)}^{2}\right)^{1 / 2} \\
\leq C \gamma \frac{\mu}{\varepsilon^{2}}|\Omega|^{1 / 2} T(k \ell)^{1 / 2} .
\end{gathered}
$$


Finally, the term involving $\delta_{t} \widetilde{\boldsymbol{d}}_{h}^{n}$ has the bound

$$
\begin{aligned}
\mu k^{2} \mid & \sum_{m=1}^{M-\ell} \sum_{n=m+1}^{m+\ell}\left(\delta_{t} \widetilde{\boldsymbol{d}}_{h}^{n}, \boldsymbol{w}_{h}^{m+\ell}-\boldsymbol{w}_{h}^{m}\right) \mid \\
& \leq \mu(k(\ell-1))^{1 / 2} T^{1 / 2}\left(\sum_{n=2}^{M} k\left\|\delta_{t} \widetilde{\boldsymbol{d}}_{h}^{n}\right\|_{\boldsymbol{L}^{2}(\Omega)}^{2}\right)^{1 / 2}\left(\sum_{m=1}^{M-\ell}\left\|\boldsymbol{w}_{h}^{m+\ell}-\boldsymbol{w}_{h}^{m}\right\|_{\boldsymbol{L}^{2}(\Omega)}^{2}\right)^{1 / 2} \\
& \leq C \mu(k \ell)^{1 / 2} T^{1 / 2},
\end{aligned}
$$

owing to (5.25) and the assumption on $\boldsymbol{l}$. Then (5.27) follows by collecting these estimates.

Remark 5.8. As stated in Remark 5.2 there are particular meshes on which the lifting $\widetilde{\boldsymbol{d}}_{h}^{n}$ satisfies the maximum principle. But extending this result to $\boldsymbol{d}_{h}^{n}$ is problematic. In particular, the steps in the proof of Lemma 3.4 cannot be applied to the discrete system (5.10), (5.11).

5.4. Weak convergence. In order to establish convergence, it is convenient to associate the following functions with the solution of (5.10), (5.11). First, let $\boldsymbol{u}_{h k} \in \mathcal{C}^{0}\left([0, T] ; \boldsymbol{H}_{0}^{1}(\Omega)\right)$ and $\boldsymbol{d}_{h k} \in \mathcal{C}^{0}\left([0, T] ; \boldsymbol{H}^{1}(\Omega)\right)$ be affine in each subinterval $\left[t_{n-1}, t_{n}\right], 1 \leq n \leq M$, with $\boldsymbol{u}_{h k}\left(t_{n}\right)=\boldsymbol{u}_{h}^{n}, \boldsymbol{d}_{h k}\left(t_{n}\right)=\boldsymbol{d}_{h}^{n}, 0 \leq n \leq M$. Next, let $\boldsymbol{u}_{h k}^{r}, \boldsymbol{u}_{h k}^{l}, \boldsymbol{d}_{h k}^{r}, \boldsymbol{d}_{h k}^{l}$ be constant in each subinterval $] t_{n-1}, t_{n}[, 1 \leq n \leq M$, with $\left.\left(\boldsymbol{u}_{h k}^{r}, \boldsymbol{d}_{h k}^{r}\right)\right|_{\left[t_{n-1}, t_{n}[\right.}=\left(\boldsymbol{u}_{h}^{n}, \boldsymbol{d}_{h}^{n}\right),\left.\left(\boldsymbol{u}_{h k}^{l}, \boldsymbol{d}_{h k}^{l}\right)\right|_{\left.] t_{n-1}, t_{n}\right]}=\left(\boldsymbol{u}_{h}^{n-1}, \boldsymbol{d}_{h}^{n-1}\right)$ respectively, $1 \leq n \leq M$. We define similarly the continuous functions $\widehat{\boldsymbol{d}}_{h k}, \widetilde{\boldsymbol{d}}_{h k}$ and the piecewise constant functions $\boldsymbol{w}_{h k}^{r}, \widehat{\boldsymbol{d}}_{h k}^{r}, \widetilde{\boldsymbol{d}}_{h k}^{r}, \widehat{\boldsymbol{d}}_{h k}^{l}$ and $\widetilde{\boldsymbol{d}}_{h k}^{l}$. Then the scheme (5.10) - (5.11) can be written in terms of these functions as follows, eliminating the pressure as usual by taking $\boldsymbol{v}$ in $V_{h}$, see (5.7):

$$
\begin{array}{r}
\forall(\boldsymbol{v}, \boldsymbol{e}) \in V_{h} \times W_{h},\left(d_{t}\left(\boldsymbol{u}_{h k}, \mu \widehat{\boldsymbol{d}}_{h k}\right),(\boldsymbol{v}, \boldsymbol{e})\right)+a\left(\left(\boldsymbol{u}_{h k}^{r}, \boldsymbol{w}_{h k}^{r}\right),(\boldsymbol{v}, \boldsymbol{e})\right) \\
+c\left(\left(\boldsymbol{u}_{h k}^{l}, \boldsymbol{d}_{h k}^{l}\right),\left(\boldsymbol{u}_{h k}^{r}, \boldsymbol{w}_{h k}^{r}\right),(\boldsymbol{v}, \boldsymbol{e})\right)=-\mu\left(\gamma \widetilde{\boldsymbol{f}}\left(\boldsymbol{d}_{h k}^{l}\right)+d_{t} \widetilde{\boldsymbol{d}}_{h k}, \boldsymbol{e}\right),
\end{array}
$$

and

$$
\forall(q, \boldsymbol{g}) \in M_{h}, b\left(\boldsymbol{u}_{h k}^{r}, q\right)+\mu\left(\nabla \widehat{\boldsymbol{d}}_{h k}^{r}, \nabla \boldsymbol{g}\right)-\left(\boldsymbol{w}_{h k}^{r}, \boldsymbol{g}\right)=0 .
$$

Lemma 5.3 implies, in particular, that the following inclusions hold with constants independent of $k$ and $h$, but depending on $\varepsilon$ :

$$
\begin{gathered}
\left(\boldsymbol{u}_{h k}^{r}, \widehat{\boldsymbol{d}}_{h k}^{r}\right),\left(\boldsymbol{u}_{h k}^{l}, \widehat{\boldsymbol{d}}_{h k}^{l}\right),\left(\boldsymbol{u}_{h k}, \widehat{\boldsymbol{d}}_{h k}\right) \text { in } L^{\infty}\left(\boldsymbol{L}^{2}(\Omega) \times \boldsymbol{H}^{1}(\Omega)\right), \\
\left(\boldsymbol{u}_{h k}^{r}, \boldsymbol{w}_{h k}^{r}\right) \text { in } L^{2}\left(\boldsymbol{H}^{1}(\Omega) \times \boldsymbol{L}^{2}(\Omega)\right) .
\end{gathered}
$$

Furthermore, it stems from (5.15) that the following inclusions are also uniform in $h$ and $k$,

$$
\boldsymbol{d}_{h k}^{r}, \boldsymbol{d}_{h k}^{l}, \boldsymbol{d}_{h k} \text { in } L^{\infty}\left(\boldsymbol{H}^{1}(\Omega)\right) .
$$

Moreover, (5.14) easily implies that

$$
\begin{aligned}
\left\|\boldsymbol{u}_{h k}^{r}-\boldsymbol{u}_{h k}^{l}, \widehat{\boldsymbol{d}}_{h k}^{r}-\widehat{\boldsymbol{d}}_{h k}^{l}\right\|_{L^{2}\left(\boldsymbol{L}^{2}(\Omega) \times \boldsymbol{H}^{1}(\Omega)\right)} \leq C k^{1 / 2}, \\
\left\|\boldsymbol{u}_{h k}^{r}-\boldsymbol{u}_{h k}, \widehat{\boldsymbol{d}}_{h k}^{r}-\widehat{\boldsymbol{d}}_{h k}\right\|_{L^{2}\left(\boldsymbol{L}^{2}(\Omega) \times \boldsymbol{H}^{1}(\Omega)\right)} \leq C k^{1 / 2} .
\end{aligned}
$$


Finally, (5.24) and (5.26) yield that the next inclusions are uniform in $h$ and $k$ :

$$
\widehat{\boldsymbol{d}}_{h k}^{r}, \boldsymbol{d}_{h k}^{r} \text { in } L^{2}\left(\boldsymbol{W}^{1,3}(\Omega)\right) .
$$

From these uniform bounds, we infer that there is a subsequence $\left(h^{\prime}, k^{\prime}\right) \subset(h, k)$, still denoted by $(h, k)$, and limit functions $\left(\boldsymbol{u}^{r}, \widehat{\boldsymbol{d}}^{r}\right),\left(\boldsymbol{u}^{l}, \widehat{\boldsymbol{d}}^{l}\right),(\boldsymbol{u}, \widehat{\boldsymbol{d}})$ and $\boldsymbol{w}$ such that

$$
\begin{gathered}
\lim _{(h, k) \rightarrow(0,0)}\left(\boldsymbol{u}_{h k}^{r}, \widehat{\boldsymbol{d}}_{h k}^{r}\right)=\left(\boldsymbol{u}^{r}, \widehat{\boldsymbol{d}}^{r}\right) \text { weakly* in } L^{\infty}\left(\boldsymbol{L}^{2}(\Omega) \times \boldsymbol{H}^{1}(\Omega)\right), \\
\lim _{(h, k) \rightarrow(0,0)}\left(\boldsymbol{u}_{h k}^{l}, \widehat{\boldsymbol{d}}_{h k}^{l}\right)=\left(\boldsymbol{u}^{l}, \widehat{\boldsymbol{d}}^{l}\right) \text { weakly* in } L^{\infty}\left(\boldsymbol{L}^{2}(\Omega) \times \boldsymbol{H}^{1}(\Omega)\right), \\
\lim _{(h, k) \rightarrow(0,0)}\left(\boldsymbol{u}_{h k}, \widehat{\boldsymbol{d}}_{h k}\right)=(\boldsymbol{u}, \widehat{\boldsymbol{d}}) \text { weakly* in } L^{\infty}\left(\boldsymbol{L}^{2}(\Omega) \times \boldsymbol{H}^{1}(\Omega)\right), \\
\lim _{(h, k) \rightarrow(0,0)}\left(\boldsymbol{u}_{h k}^{r}, \boldsymbol{w}_{h k}^{r}\right)=\left(\boldsymbol{u}^{r}, \boldsymbol{w}\right) \text { weakly in } L^{2}\left(\boldsymbol{H}^{1}(\Omega) \times \boldsymbol{L}^{2}(\Omega)\right) .
\end{gathered}
$$

Next, (5.32) implies that the limit functions satisfy

$$
\boldsymbol{u}^{r}=\boldsymbol{u}^{l}=\boldsymbol{u}, \widehat{\boldsymbol{d}}^{r}=\widehat{\boldsymbol{d}}^{l}=\widehat{\boldsymbol{d}}
$$

Similarly, the properties of the lifting sequences $\widetilde{\boldsymbol{d}}_{h k}^{r}, \widetilde{\boldsymbol{d}}_{h k}^{l}$ and $\widetilde{\boldsymbol{d}}_{h k}$, imply that this convergence can be extended to $\boldsymbol{d}_{h k}^{r}, \boldsymbol{d}_{h k}^{l}$ and $\boldsymbol{d}_{h k}$. Finally,

$$
\lim _{(h, k) \rightarrow 0} \widehat{\boldsymbol{d}}_{h k}^{r}=\widehat{\boldsymbol{d}} \text { weakly in } L^{2}\left(\boldsymbol{W}^{1,3}(\Omega)\right) .
$$

Clearly, these weak convergences together with the approximation properties of the finite element spaces $U_{h}, P_{h}, W_{h}, D_{h}$, and $V_{h}$ (for which we use the inf-sup condition), allow us to pass to the limit in all bilinear terms of (5.30) and (5.31). But passing to the limit in the non-linear terms requires compactness in space and time. While compactness in space is a straightforward consequence of Sobolev's imbeddings, proving compactness in time is much more delicate. This is the object of the next section.

\subsection{Compactness and passing to the limit. For proving that}

$$
\begin{aligned}
\lim _{(h, k) \rightarrow(0,0)} c\left(\left(\boldsymbol{u}_{h k}^{l}, \boldsymbol{d}_{h k}^{l}\right),\left(\boldsymbol{u}_{h k}^{r}, \boldsymbol{w}_{h k}^{r}\right),(\boldsymbol{v}, \boldsymbol{e})\right) & =c((\boldsymbol{u}, \boldsymbol{d}),(\boldsymbol{u}, \boldsymbol{w}),(\boldsymbol{v}, \boldsymbol{e})), \\
\lim _{(h, k) \rightarrow(0,0)}\left(\widetilde{\boldsymbol{f}}\left(\boldsymbol{d}_{h k}^{l}\right), \boldsymbol{e}\right) & =(\widetilde{\boldsymbol{f}}(\boldsymbol{d}), \boldsymbol{e}),
\end{aligned}
$$

which will lead to the conclusion that $(\boldsymbol{u}, \boldsymbol{d}, \boldsymbol{w})$ solves (4.5), (4.6) with test function $\boldsymbol{v}$ in $\boldsymbol{V}$, it suffices to show that the sequence of functions $\left(\boldsymbol{u}_{h k}^{r}, \widehat{\boldsymbol{d}}_{h k}^{r}\right)$ is compact in $L^{2}\left(\boldsymbol{L}^{2}(\Omega) \times \boldsymbol{H}^{1}(\Omega)\right)$. Indeed, in view of (5.32), the same result will be true for $\left(\boldsymbol{u}_{h k}^{l}, \widehat{\boldsymbol{d}}_{h k}^{l}\right)$ and $\left(\boldsymbol{u}_{h k}, \widehat{\boldsymbol{d}}_{h k}\right)$. This compactness argument will be split into two steps:

Step 1. Compactness of $\left(\boldsymbol{u}_{h k}^{r}, \widehat{\boldsymbol{d}}_{h k}^{r}\right)$ in $L^{2}\left(\boldsymbol{L}^{2}(\Omega) \times \boldsymbol{L}^{2}(\Omega)\right)$.

We shall use the following theorem applied to $\left(\boldsymbol{u}_{h k}^{r}, \widehat{\boldsymbol{d}}_{h k}^{r}\right)$. 
Theorem 5.9. The sequence of solutions $\left(\boldsymbol{u}_{h k}^{r}, \widehat{\boldsymbol{d}}_{h k}^{r}\right)$ is compact in $L^{2}\left(\boldsymbol{L}^{2}(\Omega) \times\right.$ $\left.\boldsymbol{L}^{2}(\Omega)\right)$ if there exists a constant $C$, independent of $h$ and $k$, such that

$$
\begin{aligned}
&\forall \delta \in] 0, T\left[, \int_{0}^{T-\delta}\right.\left\|\boldsymbol{u}_{h k}^{r}(t+\delta)-\boldsymbol{u}_{h k}^{r}(t), \mu\left(\widehat{\boldsymbol{d}}_{h k}^{r}(t+\delta)-\widehat{\boldsymbol{d}}_{h k}^{r}(t)\right)\right\|_{\boldsymbol{L}^{2}(\Omega) \times \boldsymbol{H}^{1}(\Omega)}^{2} d t \\
& \leq C \delta^{1 / 2} .
\end{aligned}
$$

Proof. Considering that the sequence $\left(\boldsymbol{u}_{h k}^{r}, \widehat{\boldsymbol{d}}_{h k}^{r}\right)$ is bounded in $L^{2}\left(\boldsymbol{H}^{1}(\Omega) \times \boldsymbol{H}^{1}(\Omega)\right)$, the Kazhikhov's type a priori fractional estimate in time (5.33) implies compactness of $\left(\boldsymbol{u}_{h k}^{r}, \widehat{\boldsymbol{d}}_{h k}^{r}\right)$ in $L^{2}\left(\boldsymbol{L}^{2}(\Omega) \times \boldsymbol{L}^{2}(\Omega)\right)$, as a consequence of a vector extension of the Kolmogorov's characterization of relatively compact sets in $L^{2}(0, T)$; see for instance [36.

The corollary below states that the a priori estimate (5.33) stems from Proposition 5.7 .

Corollary 5.10. Proposition 5.7 implies that the sequence of solutions $\left(\boldsymbol{u}_{h k}^{r}, \widehat{\boldsymbol{d}}_{h k}^{r}\right)$ is compact in $L^{2}\left(\boldsymbol{L}^{2}(\Omega) \times \boldsymbol{L}^{2}(\Omega)\right)$.

Proof. We must prove (5.33) for all real numbers $\delta \in] 0, T$ [, and it suffices to write the proof for $\boldsymbol{u}_{h k}^{r}$. The argument depends upon the value of $\delta$.

(i) If $\delta \in] 0, k\left[\right.$, then for any function $f$ in $L^{1}(0, T)$, we can write

$$
\int_{0}^{T-\delta} f(t) d t=\sum_{i=0}^{M-2}\left(\int_{t_{i}}^{t_{i+1}-\delta} f(t) d t+\int_{t_{i+1}-\delta}^{t_{i+1}} f(t) d t\right)+\int_{t_{M-1}}^{t_{M}-\delta} f(t) d t .
$$

As the functions $\boldsymbol{u}_{h k}^{r}$ are independent of time on each subinterval $\left.] t_{n-1}, t_{n}\right], 1 \leq$ $n \leq M$, this splitting leads to:

$$
\int_{0}^{T-\delta}\left\|\boldsymbol{u}_{h k}^{r}(t+\delta)-\boldsymbol{u}_{h k}^{r}(t)\right\|_{\boldsymbol{L}^{2}(\Omega)}^{2} d t=\delta \sum_{i=1}^{M-1}\left\|\boldsymbol{u}_{h}^{i+1}-\boldsymbol{u}_{h}^{i}\right\|_{\boldsymbol{L}^{2}(\Omega)}^{2} .
$$

But (5.14) implies that

$$
\delta \sum_{i=1}^{M-1}\left\|\boldsymbol{u}_{h}^{i+1}-\boldsymbol{u}_{h}^{i}\right\|_{L^{2}(\Omega)}^{2} \leq C \delta,
$$

and as $\delta<k$ and necessarily $k<1$, this implies (5.33) for $0<\delta<k$.

(ii) Now, let $1 \leq \ell \leq M-1$ and take $\delta=\ell k$. Proceeding as above and applying (5.27), we easily derive:

$$
\int_{0}^{T-\ell k}\left\|\boldsymbol{u}_{h k}^{r}(t+\ell k)-\boldsymbol{u}_{h k}^{r}(t)\right\|_{\boldsymbol{L}^{2}(\Omega)}^{2} d t=k \sum_{m=1}^{M-\ell}\left\|\boldsymbol{u}_{h}^{m+\ell}-\boldsymbol{u}_{h}^{m}\right\|_{\boldsymbol{L}^{2}(\Omega)}^{2} \leq C(\ell k)^{1 / 2} .
$$

This establishes (5.33) for $\delta=\ell k$.

(iii) Finally, consider the remaining case where $1 \leq \ell \leq M-1$ and $\delta=k(\ell+\eta)$, with $\eta \in] 0,1[$. Then the left-hand side of (5.33) splits as follows:

$$
\begin{aligned}
\int_{0}^{T-\delta} & \left\|\boldsymbol{u}_{h k}^{r}(t+\delta)-\boldsymbol{u}_{h k}^{r}(t)\right\|_{\boldsymbol{L}^{2}(\Omega)}^{2} d t \\
& =k(1-\eta) \sum_{m=1}^{M-\ell}\left\|\boldsymbol{u}_{h}^{m+\ell}-\boldsymbol{u}_{h}^{m}\right\|_{\boldsymbol{L}^{2}(\Omega)}^{2}+k \eta \sum_{m=1}^{M-(\ell+1)}\left\|\boldsymbol{u}_{h}^{m+\ell+1}-\boldsymbol{u}_{h}^{m}\right\|_{\boldsymbol{L}^{2}(\Omega)}^{2} .
\end{aligned}
$$


Applying (5.27), this becomes:

$$
\int_{0}^{T-\delta}\left\|\boldsymbol{u}_{h k}^{r}(t+\delta)-\boldsymbol{u}_{h k}^{r}(t)\right\|_{\boldsymbol{L}^{2}(\Omega)}^{2} d t \leq C k^{1 / 2}\left((1-\eta) \ell^{1 / 2}+\eta(\ell+1)^{1 / 2}\right) .
$$

But all $\eta \in] 0,1[$ and all positive integers $\ell$ satisfy

$$
(1-\eta) \ell^{1 / 2}+\eta(\ell+1)^{1 / 2} \leq \sqrt{2}(\ell+\eta)^{1 / 2},
$$

whence (5.33) holds for $\delta=k(\ell+\eta)$ and therefore in all cases.

Step 2. Compactness of $\boldsymbol{d}_{h k}^{r}$ in $L^{2}\left(\boldsymbol{H}^{1}(\Omega)\right)$.

The compactness of $\widehat{\boldsymbol{d}}_{h k}^{r}$ in $L^{2}\left(\boldsymbol{H}_{0}^{1}(\Omega)\right)$ follows from the convergence

$$
\lim _{(h, k) \rightarrow(0,0)}\left\|\widehat{\boldsymbol{d}}_{h k}^{r}\right\|_{L^{2}\left(\boldsymbol{H}_{0}^{1}(\Omega)\right)}=\|\boldsymbol{d}\|_{L^{2}\left(\boldsymbol{H}_{0}^{1}(\Omega)\right)} .
$$

Indeed, by taking $\boldsymbol{g}=\widehat{\boldsymbol{d}}_{h k}^{r}$ in (5.31), using the compactness of $\widehat{\boldsymbol{d}}_{h k}^{r}$ in $L^{2}\left(\boldsymbol{L}^{2}(\Omega)\right)$ and finally taking $\boldsymbol{g}=\boldsymbol{d}$ in (4.6), we obtain

$$
\begin{aligned}
\int_{0}^{T}\left\|\nabla \widehat{\boldsymbol{d}}_{h k}^{r}(t)\right\|_{\boldsymbol{L}^{2}(\Omega)}^{2} d t & =\int_{0}^{T}\left(\boldsymbol{w}_{h k}(t), \widehat{\boldsymbol{d}}_{h k}^{r}(t)\right) d t \rightarrow \int_{0}^{T}(\boldsymbol{w}(t), \boldsymbol{d}(t)) d t \\
& =\int_{0}^{T}\|\nabla \boldsymbol{d}(t)\|_{\boldsymbol{L}^{2}(\Omega)}^{2} d t .
\end{aligned}
$$

Then the compactness of the lifting sequence $\widetilde{\boldsymbol{d}}_{h k}^{r}$, yields the compactness of $\boldsymbol{d}_{h k}^{r}$ in $L^{2}\left(\boldsymbol{H}^{1}(\Omega)\right)$.

By combining this with the material of Section 5.4, we obtain immediately the following convergence theorem.

Theorem 5.11. The limit functions $\boldsymbol{u}, \boldsymbol{d}$ and $\boldsymbol{w}$ solve problem (4.5), (4.6) with test function $\boldsymbol{v}$ in $\boldsymbol{V}$.

Remark 5.12. As in the Navier-Stokes equations, establishing convergence of the pressure is more delicate because it involves convergence of the time derivative of the discrete velocity whose proof is fairly long and intricate; cf. Lions [27] and Temam [37. To avoid this difficulty and save space we turn directly to error estimates.

\section{ERror estimates}

In this section, we shall prove that, if the exact solution is sufficiently smooth, the error of the scheme (5.10), (5.11) (or equivalently (5.12) ) is of order one in time and space. As usual, this is achieved by inserting in the error equations suitable interpolations of the exact solution.

We shall need to approximate both the velocity and its time derivative by the same operator, and since we do not want to impose much regularity on this derivative, we need an operator that is stable in a larger space than $W^{1,1}(\Omega)$. For this, it is convenient to use the following variant $J_{h}$ of $S Z_{h}$, introduced in [14], that takes averages in elements instead of faces. Thus, for each interior node $\boldsymbol{a}$ of $\mathcal{T}_{h}$, we associate once and for all an element $T_{\boldsymbol{a}}$ of $\mathcal{T}_{h}$ with vertex $\boldsymbol{a}$, and for any function $v \in L^{1}(\Omega)$, and all interior vertices $\boldsymbol{a}$ of $\mathcal{T}_{h}$, we define

$$
J_{h} v(\boldsymbol{a})=\frac{1}{\left|T_{\boldsymbol{a}}\right|} \int_{T_{\boldsymbol{a}}} v(\boldsymbol{x}) d \boldsymbol{x} .
$$


To enforce the zero boundary value, we set $J_{h} v(\boldsymbol{a})=0$ on all boundary vertices $\boldsymbol{a}$, and we interpolate these values with $\mathbb{P}_{1}$ functions in each $T$. This operator is stable in $L^{r}(\Omega)$ for any $r$ and it has optimal approximation properties in spaces of functions that vanish on the boundary. Now, to approximate the velocity in each element $T$, we define the operator $I_{h}$ by

$$
\forall \boldsymbol{v} \in \boldsymbol{L}^{1}(T),\left.I_{h} \boldsymbol{v}\right|_{T}=\left.J_{h} \boldsymbol{v}\right|_{T}+b_{T} \boldsymbol{c}_{T}, \quad \boldsymbol{c}_{T}=\frac{1}{\int_{T} b_{T} d \boldsymbol{x}} \int_{T}\left(\boldsymbol{v}-J_{h} \boldsymbol{v}\right)(\boldsymbol{x}) d \boldsymbol{x},
$$

where $b_{T}$ is the bubble function defined in (5.2). By construction, $I_{h} \boldsymbol{v}$ belongs to $U_{0 h}$ if $\boldsymbol{v}$ belongs to $\boldsymbol{L}^{1}(\Omega)$ and it is easy to check that the approximation properties of $I_{h}$ stem directly from those of $J_{h}$. Furthermore, it satisfies

$$
\forall T \in \mathcal{T}_{h}, \forall \boldsymbol{v} \in \boldsymbol{L}^{1}(T), \int_{T}\left(\boldsymbol{v}-I_{h} \boldsymbol{v}\right)(\boldsymbol{x}) d \boldsymbol{x}=0 .
$$

This property implies on one hand that (cf. [15])

$$
\forall \boldsymbol{v} \in \boldsymbol{H}_{0}^{1}(\Omega), \forall q_{h} \in P_{h},\left(\nabla \cdot\left(\boldsymbol{v}-I_{h} \boldsymbol{v}\right), q_{h}\right)=0,
$$

which in turn implies a uniform inf-sup condition. On the other hand, (6.3) readily yields that

$$
\forall \boldsymbol{v} \in \boldsymbol{L}^{2}(\Omega),\left\|\boldsymbol{v}-I_{h} \boldsymbol{v}\right\|_{H^{-1}(\Omega)} \leq C h\left\|\boldsymbol{v}-I_{h} \boldsymbol{v}\right\|_{L^{2}(\Omega)} .
$$

We approximate the director field and the pressure with the regularization operator $S Z_{h}$ defined in (5.8). Strictly speaking $S Z_{h}$ does not preserve the zero mean value in $\Omega$, but it is easy to correct it without changing its approximation properties. To avoid a multiplicity of notation, we still denote the corrected operator by $S Z_{h}$. Finally, we approximate the auxiliary field $\boldsymbol{w}$ by the $L^{2}$ projection operator $K_{h}$ on constants in each $T$ :

$$
\forall T \in \mathcal{T}_{h}, \forall w \in L^{2}(T),\left.K_{h} w\right|_{T}=\frac{1}{|T|} \int_{T} w(\boldsymbol{x}) d \boldsymbol{x} .
$$

In addition to (6.5), we shall use the following approximation and stability properties of $I_{h}, S Z_{h}$ and $K_{h}$, valid for any number $p \in[1, \infty]$ :

$$
\begin{gathered}
\left\|I_{h} v\right\|_{W^{n, p}(\Omega)} \leq C\|v\|_{W^{n, p}(\Omega)} \quad n=0,1 \\
\left\|I_{h} v-v\right\|_{W^{n, p}(\Omega)} \leq C h|v|_{W^{n+1, p}(\Omega)} \quad n=0,1 \\
\left\|S Z_{h} d-d\right\|_{W^{n, p}(\Omega)} \leq C h|d|_{W^{n+1, p}(\Omega)} \quad n=0,1 \\
\left\|K_{h} w\right\|_{L^{p}(\Omega)} \leq C\|w\|_{L^{p}(\Omega)}, \quad\left\|K_{h} w-w\right\|_{L^{p}(\Omega)} \leq C h|w|_{W^{1, p}(\Omega)}, \\
\left\|S Z_{h} p-p\right\|_{L^{2}(\Omega)} \leq C h|p|_{H^{1}(\Omega)} .
\end{gathered}
$$

With these operators, we define the (fully discrete) errors

$$
\boldsymbol{e}_{\boldsymbol{u}}^{n}:=\boldsymbol{u}_{h}^{n}-I_{h} \boldsymbol{u}\left(t_{n}\right), \quad \boldsymbol{e}_{\boldsymbol{d}}^{n}:=\boldsymbol{d}_{h}^{n}-S Z_{h} \boldsymbol{d}\left(t_{n}\right), \quad \boldsymbol{e}_{\boldsymbol{w}}^{n}:=\boldsymbol{w}_{h}^{n}-K_{h} \boldsymbol{w}\left(t_{n}\right) .
$$

6.1. Consistency errors. By integrating (4.5) with respect to time between $t_{n-1}$ and $t_{n}$, by taking (4.6) at time $t=t_{n}$, and by subtracting (5.12), we obtain the 
error equations respectively for all $\left(\boldsymbol{v}_{h}, \boldsymbol{e}_{h}\right) \in X_{h}$ and $\left(q_{h}, \boldsymbol{g}_{h}\right) \in M_{h}$ :

$$
\begin{aligned}
& k \delta_{t}\left(\left(\boldsymbol{u}\left(t_{n}\right)-\boldsymbol{u}_{h}^{n}, \mu\left(\boldsymbol{d}\left(t_{n}\right)-\boldsymbol{d}_{h}^{n}\right)\right),\left(\boldsymbol{v}_{h}, \boldsymbol{e}_{h}\right)\right) \\
& +\int_{t_{n-1}}^{t_{n}} a\left(\left(\boldsymbol{u}(t)-\boldsymbol{u}_{h}^{n}, \boldsymbol{w}(t)-\boldsymbol{w}_{h}^{n}\right),\left(\boldsymbol{v}_{h}, \boldsymbol{e}_{h}\right)\right) d t+\int_{t_{n-1}}^{t_{n}} b\left(\boldsymbol{v}_{h}, p(t)-p_{h}^{n}\right) d t \\
& +\int_{t_{n-1}}^{t_{n}}\left(c\left((\boldsymbol{u}(t), \boldsymbol{d}(t)),(\boldsymbol{u}(t), \boldsymbol{w}(t)),\left(\boldsymbol{v}_{h}, \boldsymbol{e}_{h}\right)\right)\right. \\
& \left.\quad-c\left(\left(\boldsymbol{u}_{h}^{n-1}, \boldsymbol{d}_{h}^{n-1}\right),\left(\boldsymbol{u}_{h}^{n}, \boldsymbol{w}_{h}^{n}\right),\left(\boldsymbol{v}_{h}, \boldsymbol{e}_{h}\right)\right)\right) d t \\
& +\mu \gamma \int_{t_{n-1}}^{t_{n}}\left(\widetilde{\boldsymbol{f}}(\boldsymbol{d}(t))-\widetilde{\boldsymbol{f}}\left(\boldsymbol{d}_{h}^{n-1}\right), \boldsymbol{e}_{h}\right) d t=0, \\
& b\left(\boldsymbol{u}\left(t_{n}\right)-\boldsymbol{u}_{h}^{n}, q_{h}\right)+\mu\left(\nabla\left(\boldsymbol{d}\left(t_{n}\right)-\boldsymbol{d}_{h}^{n}\right), \nabla \boldsymbol{g}_{h}\right)-\left(\boldsymbol{w}\left(t_{n}\right)-\boldsymbol{w}_{h}^{n}, \boldsymbol{g}_{h}\right)=0 .
\end{aligned}
$$

This system involves the following consistency error terms (the derivative with respect to time is denoted by a prime):

$$
\begin{aligned}
& \mathcal{E}_{h, 1}^{n}\left(\boldsymbol{v}_{h}, \boldsymbol{e}_{h}\right)=\int_{t_{n-1}}^{t_{n}}\left(\left(\boldsymbol{u}^{\prime}(t)-I_{h} \boldsymbol{u}^{\prime}(t), \mu\left(\boldsymbol{d}^{\prime}(t)-S Z_{h} \boldsymbol{d}^{\prime}(t)\right)\right),\left(\boldsymbol{v}_{h}, \boldsymbol{e}_{h}\right)\right) d t, \\
& \mathcal{E}_{h, a}^{n}\left(\boldsymbol{v}_{h}, \boldsymbol{e}_{h}\right)=\mathcal{E}_{h, a, 1}^{n}\left(\boldsymbol{v}_{h}, \boldsymbol{e}_{h}\right)+\mathcal{E}_{h, a, 2}^{n}\left(\boldsymbol{v}_{h}, \boldsymbol{e}_{h}\right) \\
& \quad \int_{t_{n-1}}^{t_{n}} a\left(\left(\boldsymbol{u}(t)-I_{h} \boldsymbol{u}(t), \boldsymbol{w}(t)-K_{h} \boldsymbol{w}(t)\right),\left(\boldsymbol{v}_{h}, \boldsymbol{e}_{h}\right)\right) d t \\
& \quad+\int_{t_{n-1}}^{t_{n}} a\left(\left(I_{h}\left(\boldsymbol{u}(t)-\boldsymbol{u}\left(t_{n}\right)\right), K_{h}\left(\boldsymbol{w}(t)-\boldsymbol{w}\left(t_{n}\right)\right)\right),\left(\boldsymbol{v}_{h}, \boldsymbol{e}_{h}\right)\right) d t, \\
& \mathcal{E}_{h, b}^{n}\left(\boldsymbol{v}_{h}\right)=\int_{t_{n-1}}^{t_{n}}\left(p(t)-S Z_{h} p(t), \nabla \cdot \boldsymbol{v}_{h}\right) d t \\
& \quad+\int_{t_{n-1}}^{t_{n}}\left(S Z_{h}\left(p(t)-p\left(t_{n}\right)\right), \nabla \cdot \boldsymbol{v}_{h}\right) d t, \\
& \mathcal{E}_{h, f}^{n}\left(\boldsymbol{e}_{h}\right)=\mathcal{E}_{h,, 1}^{n}\left(\boldsymbol{e}_{h}\right)+\mathcal{E}_{h, f, 2}^{n}\left(\boldsymbol{e}_{h}\right) \\
& \quad \mu \gamma \int_{t_{n-1}}^{t_{n}}\left(\widetilde{\boldsymbol{f}}(\boldsymbol{d}(t))-\widetilde{\boldsymbol{f}}\left(S Z_{h} \boldsymbol{d}(t)\right), \boldsymbol{e}_{h}\right) d t \\
& \quad+\mu \gamma \int_{t_{n-1}}^{t_{n}}\left(\widetilde{\boldsymbol{f}}\left(S Z_{h} \boldsymbol{d}(t)\right)-\widetilde{\boldsymbol{f}}\left(S Z_{h} \boldsymbol{d}\left(t_{n-1}\right)\right), \boldsymbol{e}_{h}\right) d t, \\
& \mathcal{E}_{h, c}^{n}\left(\boldsymbol{v}_{h}, \boldsymbol{e}_{h}\right)=\mathcal{E}_{h, c, 1}^{n}\left(\boldsymbol{v}_{h}, \boldsymbol{e}_{h}\right)+\mathcal{E}_{h, c, 2}^{n}\left(\boldsymbol{v}_{h}, \boldsymbol{e}_{h}\right)+\mathcal{E}_{h, c, 3}^{n}\left(\boldsymbol{v}_{h}, \boldsymbol{e}_{h}\right)+\mathcal{E}_{h, c, 4}^{n}\left(\boldsymbol{v}_{h}, \boldsymbol{e}_{h}\right) \\
& \quad=\int_{t_{n-1}}^{t_{n}} c\left((\boldsymbol{u}(t), \boldsymbol{d}(t)),\left(\boldsymbol{u}(t)-I_{h} \boldsymbol{u}(t), \boldsymbol{w}(t)-K_{h} \boldsymbol{w}(t)\right),\left(\boldsymbol{v}_{h}, \boldsymbol{e}_{h}\right)\right) d t \\
& \quad+\int_{t_{n-1}}^{t_{n}} c\left((\boldsymbol{u}(t), \boldsymbol{d}(t)),\left(I_{h}\left(\boldsymbol{u}(t)-\boldsymbol{u}\left(t_{n}\right)\right), K_{h}\left(\boldsymbol{w}(t)-\boldsymbol{w}\left(t_{n}\right)\right)\right),\left(\boldsymbol{v}_{h}, \boldsymbol{e}_{h}\right)\right) d t \\
& \quad+\int_{t_{n-1}}^{t_{n}} c\left(\left(\boldsymbol{u}(t)-I_{h} \boldsymbol{u}(t), \boldsymbol{d}(t)-S Z_{h} \boldsymbol{d}(t)\right),\left(I_{h} \boldsymbol{u}\left(t_{n}\right), K_{h} \boldsymbol{w}\left(t_{n}\right)\right),\left(\boldsymbol{v}_{h}, \boldsymbol{e}_{h}\right)\right) d t \\
& \quad
\end{aligned}
$$




$$
\begin{gathered}
+\int_{t_{n-1}}^{t_{n}} c\left(\left(I_{h}\left(\boldsymbol{u}(t)-\boldsymbol{u}\left(t_{n-1}\right)\right), S Z_{h}\left(\boldsymbol{d}(t)-\boldsymbol{d}\left(t_{n-1}\right)\right)\right),\right. \\
\left.\left(I_{h} \boldsymbol{u}\left(t_{n}\right), K_{h} \boldsymbol{w}\left(t_{n}\right)\right),\left(\boldsymbol{v}_{h}, \boldsymbol{e}_{h}\right)\right) d t, \\
\widetilde{\mathcal{E}}_{h}^{n}\left(\boldsymbol{g}_{h}\right)=\mu\left(\nabla\left(\boldsymbol{d}\left(t_{n}\right)-S Z_{h} \boldsymbol{d}\left(t_{n}\right)\right), \nabla \boldsymbol{g}_{h}\right)-\left(\boldsymbol{w}\left(t_{n}\right)-K_{h} \boldsymbol{w}\left(t_{n}\right), \boldsymbol{g}_{h}\right) .
\end{gathered}
$$

Note that on one hand the second term in $\mathcal{E}_{h, b}^{n}\left(\boldsymbol{v}_{h}\right)$ vanishes because $\boldsymbol{v}_{h}$ belongs to $V_{h}$, and on the other hand, we use (6.4) in the definition of $\widetilde{\mathcal{E}}_{h}^{n}\left(\boldsymbol{g}_{h}\right)$.

Let us bound each term, assuming that the solution is sufficiently smooth. To simplify the notation, we drop the dependence on $\boldsymbol{v}_{h}$ and $\boldsymbol{e}_{h}$ and we set $I_{n}=$ $\left(t_{n-1}, t_{n}\right)$. For estimating $\mathcal{E}_{h, 1}^{n}$, we apply (6.5) and the first part of (6.7) with $n=0, p=2$ :

$$
\left|\int_{t_{n-1}}^{t_{n}}\left(\boldsymbol{u}^{\prime}(t)-I_{h} \boldsymbol{u}^{\prime}(t), \boldsymbol{v}_{h}\right) d t\right| \leq C h\left\|\boldsymbol{u}^{\prime}\right\|_{L^{2}\left(I_{n} ; \boldsymbol{L}^{2}(\Omega)\right)} k^{1 / 2}\left|\boldsymbol{v}_{h}\right|_{\boldsymbol{H}^{1}(\Omega)}
$$

and we apply (6.8) with $n=0, p=2$ :

$$
\left|\int_{t_{n-1}}^{t_{n}}\left(\boldsymbol{d}^{\prime}(t)-S Z_{h} \boldsymbol{d}^{\prime}(t), \boldsymbol{e}_{h}\right) d t\right| \leq C h\left\|\boldsymbol{d}^{\prime}\right\|_{L^{2}\left(I_{n} ; \boldsymbol{H}^{1}(\Omega)\right)} k^{1 / 2}\left\|\boldsymbol{e}_{h}\right\|_{\boldsymbol{L}^{2}(\Omega)} .
$$

Thus

$$
\left|\mathcal{E}_{h, 1}^{n}\right| \leq C h k^{1 / 2}\left\|\boldsymbol{u}^{\prime}, \mu \boldsymbol{d}^{\prime}\right\|_{L^{2}\left(I_{n} ; \boldsymbol{L}^{2}(\Omega) \times \boldsymbol{H}^{1}(\Omega)\right)}\left\|\boldsymbol{v}_{h}, \boldsymbol{e}_{h}\right\|_{X} .
$$

Similarly, by applying the second part of (6.7) with $n=1, p=2$ and (6.9), we obtain

$$
\left|\mathcal{E}_{h, a, 1}^{n}\right| \leq C h k^{1 / 2}\|\nu \boldsymbol{u}, \gamma \boldsymbol{w}\|_{L^{2}\left(I_{n} ; \boldsymbol{H}^{2}(\Omega) \times \boldsymbol{H}^{1}(\Omega)\right)}\left\|\boldsymbol{v}_{h}, \boldsymbol{e}_{h}\right\|_{X} .
$$

To estimate the second term in $\mathcal{E}_{h, a}^{n}$, we consider, for instance,

$$
\begin{aligned}
& \int_{t_{n-1}}^{t_{n}}\left(\nabla I_{h}\left(\boldsymbol{u}(t)-\boldsymbol{u}\left(t_{n}\right)\right), \nabla \boldsymbol{v}_{h}\right) d t=\left(\nabla I_{h} \int_{t_{n-1}}^{t_{n}}\left(\boldsymbol{u}(t)-\boldsymbol{u}\left(t_{n}\right)\right) d t, \nabla \boldsymbol{v}_{h}\right) \\
& =-\left(\nabla I_{h} \int_{t_{n-1}}^{t_{n}}\left(\int_{t}^{t_{n}} \boldsymbol{u}^{\prime}(s) d s\right) d t, \nabla \boldsymbol{v}_{h}\right)=-\int_{t_{n-1}}^{t_{n}}\left(s-t_{n-1}\right)\left(\nabla I_{h} \boldsymbol{u}^{\prime}(s), \nabla \boldsymbol{v}_{h}\right) d s .
\end{aligned}
$$

Thus, applying the first part of (6.7) with $n=1, p=2$, we derive:

$$
\left|\int_{t_{n-1}}^{t_{n}}\left(\nabla I_{h}\left(\boldsymbol{u}(t)-\boldsymbol{u}\left(t_{n}\right)\right), \nabla \boldsymbol{v}_{h}\right) d t\right| \leq \frac{C}{\sqrt{3}} k^{3 / 2}\left\|\boldsymbol{u}^{\prime}\right\|_{L^{2}\left(I_{n} ; \boldsymbol{H}^{1}(\Omega)\right)}\left|\boldsymbol{v}_{h}\right|_{\boldsymbol{H}^{1}(\Omega)} .
$$

Hence,

$$
\left|\mathcal{E}_{h, a, 2}^{n}\right| \leq \frac{C}{\sqrt{3}} k^{3 / 2}\left\|\nu \boldsymbol{u}^{\prime}, \gamma \boldsymbol{w}^{\prime}\right\|_{L^{2}\left(I_{n} ; \boldsymbol{H}^{1}(\Omega) \times \boldsymbol{L}^{2}(\Omega)\right)}\left\|\boldsymbol{v}_{h}, \boldsymbol{e}_{h}\right\|_{X} .
$$

Similarly,

$$
\left|\mathcal{E}_{h, b}^{n}\right| \leq C h\|p\|_{L^{2}\left(I_{n} ; H^{1}(\Omega)\right)} k^{1 / 2}\left|\boldsymbol{v}_{h}\right|_{\boldsymbol{H}^{1}(\Omega)} .
$$

Now, we turn to the non-linear terms. First, considering that $|\boldsymbol{d}| \leq 1$ and the operator $S Z_{h}$ preserves this property, we apply formula (8.1) in the Appendix and obtain

$$
\left|\widetilde{\boldsymbol{f}}(\boldsymbol{d}(t))-\widetilde{\boldsymbol{f}}\left(S Z_{h} \boldsymbol{d}(t)\right)\right| \leq \frac{3}{\varepsilon^{2}}\left|\boldsymbol{d}(t)-S Z_{h} \boldsymbol{d}(t)\right| .
$$


Applying (6.8) with $n=0, p=2$, yields

$$
\left|\mathcal{E}_{h, f, 1}^{n}\right| \leq \mu \gamma \frac{3}{\varepsilon^{2}} C h\|\boldsymbol{d}\|_{L^{2}\left(I_{n} ; \boldsymbol{H}^{1}(\Omega)\right)} k^{1 / 2}\left\|\boldsymbol{e}_{h}\right\|_{\boldsymbol{L}^{2}(\Omega)} .
$$

For the second term in $\mathcal{E}_{h, f}^{n}$, by applying again formula (8.1), we have

$$
\left|\widetilde{\boldsymbol{f}}\left(S Z_{h} \boldsymbol{d}(t)\right)-\tilde{\boldsymbol{f}}\left(S Z_{h} \boldsymbol{d}\left(t_{n-1}\right)\right)\right| \leq \frac{3}{\varepsilon^{2}}\left|S Z_{h}\left(\boldsymbol{d}(t)-\boldsymbol{d}\left(t_{n-1}\right)\right)\right| .
$$

Therefore, proceeding as in the derivation of (6.15), we obtain

$$
\left|\mathcal{E}_{h, f, 2}^{n}\right| \leq \mu \gamma \frac{\sqrt{3}}{\varepsilon^{2}} C\left\|\boldsymbol{d}^{\prime}\right\|_{L^{2}\left(I_{n} ; \boldsymbol{H}^{1}(\Omega)\right)} k^{3 / 2}\left\|\boldsymbol{e}_{h}\right\|_{\boldsymbol{L}^{2}(\Omega)} .
$$

Therefore,

$$
\left|\mathcal{E}_{h, f}^{n}\right| \leq \mu \gamma \frac{C}{\varepsilon^{2}} k^{1 / 2}\left(3 h\|\boldsymbol{d}\|_{L^{2}\left(I_{n} ; \boldsymbol{H}^{1}(\Omega)\right)}+\sqrt{3} k\left\|\boldsymbol{d}^{\prime}\right\|_{L^{2}\left(I_{n} ; \boldsymbol{H}^{1}(\Omega)\right)}\right)\left\|\boldsymbol{e}_{h}\right\|_{\boldsymbol{L}^{2}(\Omega)} .
$$

It remains to consider the terms involving trilinear form $c$. First, as $\boldsymbol{u}(t)$ belongs to $\boldsymbol{V}$, we write the first term of $\mathcal{E}_{h, a, 1}^{n}$ as follows:

$$
\int_{t_{n-1}}^{t_{n}}\left(\boldsymbol{u}(t) \cdot \nabla\left(\boldsymbol{u}(t)-I_{h} \boldsymbol{u}(t)\right), \boldsymbol{v}_{h}\right) d t=-\int_{t_{n-1}}^{t_{n}}\left(\boldsymbol{u}(t) \cdot \nabla \boldsymbol{v}_{h}, \boldsymbol{u}(t)-I_{h} \boldsymbol{u}(t)\right) d t .
$$

Therefore, applying the second part of (6.7) with $n=0, p=3$ and Sobolev's imbedding, we obtain

$$
\begin{aligned}
& \quad\left|\int_{t_{n-1}}^{t_{n}}\left(\boldsymbol{u}(t) \cdot \nabla\left(\boldsymbol{u}(t)-I_{h} \boldsymbol{u}(t)\right), \boldsymbol{v}_{h}\right) d t\right| \\
& \quad \leq C h k^{1 / 2}\|\boldsymbol{u}\|_{L^{\infty}\left(I_{n} ; \boldsymbol{H}^{1}(\Omega)\right)}\|\boldsymbol{u}\|_{L^{2}\left(I_{n} ; \boldsymbol{W}^{1,3}(\Omega)\right)}\left|\boldsymbol{v}_{h}\right|_{\boldsymbol{H}^{1}(\Omega)} .
\end{aligned}
$$

For the second term in $\mathcal{E}_{h, c, 1}^{n}$, we use the second part of (6.7) with $n=0, p=6$ and (6.9) with $p=2$ :

$$
\begin{gathered}
\left|\int_{t_{n-1}}^{t_{n}}\left(\left(\boldsymbol{u}(t)-I_{h} \boldsymbol{u}(t)\right) \cdot \nabla \boldsymbol{d}(t), \boldsymbol{e}_{h}\right) d t\right|+\left|\int_{t_{n-1}}^{t_{n}}\left(\boldsymbol{v}_{h} \cdot \nabla \boldsymbol{d}(t), \boldsymbol{w}(t)-K_{h} \boldsymbol{w}(t)\right) d t\right| \\
\leq C h k^{1 / 2}\|\boldsymbol{d}\|_{L^{\infty}\left(I_{n} ; \boldsymbol{W}^{1,3}(\Omega)\right)}\|\boldsymbol{u}, \boldsymbol{w}\|_{L^{2}\left(I_{n} ; \boldsymbol{W}^{1,6}(\Omega) \times \boldsymbol{H}^{1}(\Omega)\right)}\left\|\boldsymbol{v}_{h}, \boldsymbol{e}_{h}\right\|_{X} .
\end{gathered}
$$

The sum of these two bounds gives

$$
\begin{aligned}
\left|\mathcal{E}_{h, c, 1}^{n}\right| \leq C h k^{1 / 2}( & \mu\left\|\boldsymbol{e}_{h}\right\|_{\boldsymbol{L}^{2}(\Omega)}\|\boldsymbol{d}\|_{L^{\infty}\left(I_{n} ; \boldsymbol{W}^{1,3}(\Omega)\right)}\|\boldsymbol{u}\|_{L^{2}\left(I_{n} ; \boldsymbol{W}^{1,6}(\Omega)\right)} \\
& +\left|\boldsymbol{v}_{h}\right|_{\boldsymbol{H}^{1}(\Omega)}\left(\|\boldsymbol{u}\|_{L^{\infty}\left(I_{n} ; \boldsymbol{H}^{1}(\Omega)\right)}\|\boldsymbol{u}\|_{L^{2}\left(I_{n} ; \boldsymbol{W}^{1,3}(\Omega)\right)}\right. \\
& \left.\left.+\mu\|\boldsymbol{d}\|_{L^{\infty}\left(I_{n} ; \boldsymbol{W}^{1,3}(\Omega)\right)}\|\boldsymbol{w}\|_{L^{2}\left(I_{n} ; \boldsymbol{H}^{1}(\Omega)\right)}\right)\right) .
\end{aligned}
$$

Next, proceeding as in the derivation of (6.15), we write

$$
\int_{t_{n-1}}^{t_{n}}\left(\boldsymbol{u}(t) \cdot \nabla I_{h}\left(\boldsymbol{u}(t)-\boldsymbol{u}\left(t_{n}\right)\right), \boldsymbol{v}_{h}\right) d t=-\int_{t_{n-1}}^{t_{n}}\left(\boldsymbol{u}(t) \cdot \nabla \boldsymbol{v}_{h}, \int_{t_{n}}^{t} I_{h} \boldsymbol{u}^{\prime}(s) d s\right) d t .
$$

Hence, applying the first part of (6.7) with $n=0, p=3$, we have

$$
\begin{aligned}
& \left|\int_{t_{n-1}}^{t_{n}}\left(\boldsymbol{u}(t) \cdot \nabla I_{h}\left(\boldsymbol{u}(t)-\boldsymbol{u}\left(t_{n}\right)\right), \boldsymbol{v}_{h}\right) d t\right| \\
& \quad \leq \frac{C}{\sqrt{3}} k^{3 / 2}\|\boldsymbol{u}\|_{L^{\infty}\left(I_{n} ; \boldsymbol{H}^{1}(\Omega)\right)}\left\|\boldsymbol{u}^{\prime}\right\|_{L^{2}\left(I_{n} ; \boldsymbol{L}^{3}(\Omega)\right)}\left|\boldsymbol{v}_{h}\right|_{\boldsymbol{H}^{1}(\Omega)} .
\end{aligned}
$$


Similarly, arguing as in the proof of (6.15), we have the following bound for the second term in $\mathcal{E}_{h, c, 2}^{n}$ :

$$
\begin{aligned}
\mid \int_{t_{n-1}}^{t_{n}}\left(I_{h}\left(\boldsymbol{u}(t)-\boldsymbol{u}\left(t_{n}\right)\right)\right. & \left.\cdot \nabla \boldsymbol{d}(t), \boldsymbol{e}_{h}\right) d t|+| \int_{t_{n-1}}^{t_{n}}\left(\boldsymbol{v}_{h} \cdot \nabla \boldsymbol{d}(t), K_{h}\left(\boldsymbol{w}(t)-\boldsymbol{w}\left(t_{n}\right)\right)\right) d t \mid \\
& \leq \frac{C}{\sqrt{3}} k^{3 / 2}\left(\|\boldsymbol{d}\|_{L^{\infty}\left(I_{n} ; \boldsymbol{W}^{1,6}(\Omega)\right)}\left\|\boldsymbol{u}^{\prime}\right\|_{L^{2}\left(I_{n} ; \boldsymbol{L}^{3}(\Omega)\right)}\left\|\boldsymbol{e}_{h}\right\|_{\boldsymbol{L}^{2}(\Omega)}\right. \\
& \left.+\|\boldsymbol{d}\|_{L^{\infty}\left(I_{n} ; \boldsymbol{W}^{1,3}(\Omega)\right)}\left\|\boldsymbol{w}^{\prime}\right\|_{L^{2}\left(\Omega \times I_{n}\right)}\left|\boldsymbol{v}_{h}\right|_{\boldsymbol{H}^{1}(\Omega)}\right) .
\end{aligned}
$$

Hence,

$$
\begin{aligned}
\left|\mathcal{E}_{h, c, 2}^{n}\right| \leq \frac{C}{\sqrt{3}} k^{3 / 2}( & \mu\left\|\boldsymbol{e}_{h}\right\|_{\boldsymbol{L}^{2}(\Omega)}\|\boldsymbol{d}\|_{L^{\infty}\left(I_{n} ; \boldsymbol{W}^{1,6}(\Omega)\right)}\left\|\boldsymbol{u}^{\prime}\right\|_{L^{2}\left(I_{n} ; \boldsymbol{L}^{3}(\Omega)\right)} \\
& +\left|\boldsymbol{v}_{h}\right|_{\boldsymbol{H}^{1}(\Omega)}\left(\|\boldsymbol{u}\|_{L^{\infty}\left(I_{n} ; \boldsymbol{H}^{1}(\Omega)\right)}\left\|\boldsymbol{u}^{\prime}\right\|_{L^{2}\left(I_{n} ; \boldsymbol{L}^{3}(\Omega)\right)}\right. \\
& \left.\left.+\mu\|\boldsymbol{d}\|_{L^{\infty}\left(I_{n} ; \boldsymbol{W}^{1,3}(\Omega)\right)}\left\|\boldsymbol{w}^{\prime}\right\|_{L^{2}\left(\Omega \times I_{n}\right)}\right)\right)
\end{aligned}
$$

Next, the bound for $\mathcal{E}_{h, c, 3}^{n}$ is a straightforward variant of (6.19), and we skip the details:

$$
\begin{aligned}
\left|\mathcal{E}_{h, c, 3}^{n}\right| \leq C h k^{1 / 2}( & \mu\left\|\boldsymbol{e}_{h}\right\|_{\boldsymbol{L}^{2}(\Omega)}\|\boldsymbol{u}\|_{L^{\infty}\left(I_{n} ; \boldsymbol{H}^{1}(\Omega)\right)}\|\boldsymbol{d}\|_{L^{2}\left(I_{n} ; \boldsymbol{W}^{2,3}(\Omega)\right)} \\
& +\left|\boldsymbol{v}_{h}\right|_{\boldsymbol{H}^{1}(\Omega)}\left(\|\boldsymbol{u}\|_{L^{\infty}\left(I_{n} ; \boldsymbol{H}^{1}(\Omega)\right)}\|\boldsymbol{u}\|_{L^{2}\left(I_{n} ; \boldsymbol{W}^{1,3}(\Omega)\right)}\right. \\
& \left.\left.+\mu\|\boldsymbol{w}\|_{L^{\infty}\left(I_{n} ; \boldsymbol{L}^{2}(\Omega)\right)}\|\boldsymbol{d}\|_{L^{2}\left(I_{n} ; \boldsymbol{W}^{2,3}(\Omega)\right)}\right)\right) .
\end{aligned}
$$

Similarly, the bound for $\mathcal{E}_{h, c, 4}^{n}$ is a straightforward variant of (6.20)

$$
\begin{aligned}
\left|\mathcal{E}_{h, c, 4}^{n}\right| \leq \frac{C}{\sqrt{3}} k^{3 / 2}( & \mu\left\|\boldsymbol{e}_{h}\right\|_{\boldsymbol{L}^{2}(\Omega)}\|\boldsymbol{u}\|_{L^{\infty}\left(I_{n} ; \boldsymbol{H}^{1}(\Omega)\right)}\left\|\boldsymbol{d}^{\prime}\right\|_{L^{2}\left(I_{n} ; \boldsymbol{W}^{1,3}(\Omega)\right)} \\
& +\left|\boldsymbol{v}_{h}\right|_{\boldsymbol{H}^{1}(\Omega)}\left(\|\boldsymbol{u}\|_{L^{\infty}\left(I_{n} ; \boldsymbol{H}^{1}(\Omega)\right)}\left\|\boldsymbol{u}^{\prime}\right\|_{L^{2}\left(I_{n} ; \boldsymbol{L}^{3}(\Omega)\right)}\right. \\
& \left.\left.+\mu\left\|\boldsymbol{d}^{\prime}\right\|_{L^{2}\left(I_{n} ; \boldsymbol{W}^{1,3}(\Omega)\right)}\|\boldsymbol{w}\|_{L^{\infty}\left(I_{n} ; \boldsymbol{L}^{2}(\Omega)\right)}\right)\right) .
\end{aligned}
$$

Finally, we easily derive a bound for $\widetilde{\mathcal{E}}_{h}^{n}$, applying (6.8) with $p=2, n=1$ and the second part of (6.9) with $p=6 / 5$ :

$$
\left|\widetilde{\mathcal{E}}_{h}^{n}\left(\boldsymbol{g}_{h}\right)\right| \leq C h\left|\boldsymbol{g}_{h}\right|_{\boldsymbol{H}^{1}(\Omega)}\left(\mu\left\|\boldsymbol{d}\left(t_{n}\right)\right\|_{\boldsymbol{H}^{2}(\Omega)}+\left\|\boldsymbol{w}\left(t_{n}\right)\right\|_{\boldsymbol{W}^{1,6 / 5}(\Omega)}\right) .
$$

6.2. Total error estimates. Let $\mathcal{E}_{h}^{n}$ denote the sum of the consistency errors in the first system:

$$
\mathcal{E}_{h}^{n}\left(\boldsymbol{v}_{h}, \boldsymbol{e}_{h}\right)=\mathcal{E}_{h, 1}^{n}\left(\boldsymbol{v}_{h}, \boldsymbol{e}_{h}\right)+\mathcal{E}_{h, a}^{n}\left(\boldsymbol{v}_{h}, \boldsymbol{e}_{h}\right)+\mathcal{E}_{h, b}^{n}\left(\boldsymbol{v}_{h}\right)+\mathcal{E}_{h, f}^{n}\left(\boldsymbol{e}_{h}\right)+\mathcal{E}_{h, c}^{n}\left(\boldsymbol{v}_{h}, \boldsymbol{e}_{h}\right) .
$$

Then the error equations (6.11), (6.12) can be expressed in terms of the total errors $e_{u}^{n}, e_{d}^{n}, e_{w}^{n}$ :

$$
\left\{\begin{aligned}
\forall & \left(\boldsymbol{v}_{h}, \boldsymbol{e}_{h}\right) \in V_{h} \times W_{h}, \\
& k\left(\delta_{t}\left(\boldsymbol{e}_{\boldsymbol{u}}^{n}, \mu \boldsymbol{e}_{\boldsymbol{d}}^{n}\right),\left(\boldsymbol{v}_{h}, \boldsymbol{e}_{h}\right)\right)+k a\left(\left(\boldsymbol{e}_{\boldsymbol{u}}^{n}, \boldsymbol{e}_{\boldsymbol{w}}^{n}\right),\left(\boldsymbol{v}_{h}, \boldsymbol{e}_{h}\right)\right) \\
+ & k c\left(\left(I_{h} \boldsymbol{u}\left(t_{n-1}\right), S Z_{h} \boldsymbol{d}\left(t_{n-1}\right)\right),\left(\boldsymbol{e}_{\boldsymbol{u}}^{n}, \boldsymbol{e}_{\boldsymbol{w}}^{n}\right),\left(\boldsymbol{v}_{h}, \boldsymbol{e}_{h}\right)\right) \\
+ & k c\left(\left(\boldsymbol{e}_{\boldsymbol{u}}^{n-1}, \boldsymbol{e}_{\boldsymbol{d}}^{n-1}\right),\left(\boldsymbol{u}_{h}^{n}, \boldsymbol{w}_{h}^{n}\right),\left(\boldsymbol{v}_{h}, \boldsymbol{e}_{h}\right)\right) \\
& =\mathcal{E}_{h}^{n}\left(\boldsymbol{v}_{h}, \boldsymbol{e}_{h}\right)-k \mu \gamma\left(\widetilde{\boldsymbol{f}}\left(\boldsymbol{d}_{h}^{n-1}\right)-\widetilde{\boldsymbol{f}}\left(S Z_{h} \boldsymbol{d}\left(t_{n-1}\right)\right), \boldsymbol{e}_{h}\right),
\end{aligned}\right.
$$




$$
\forall \boldsymbol{g}_{h} \in \boldsymbol{D}_{0 h}, k \mu\left(\nabla \boldsymbol{e}_{\boldsymbol{d}}^{n}, \nabla \boldsymbol{g}_{h}\right)-k\left(\boldsymbol{e}_{\boldsymbol{w}}^{n}, \boldsymbol{g}_{h}\right)=k \widetilde{\mathcal{E}}_{h}^{n}\left(\boldsymbol{g}_{h}\right) .
$$

Note that there is no pressure error term in the left-hand side of (6.24) because $\boldsymbol{v}_{h}$ belongs to $V_{h}$ and similarly, there is no velocity error term in the left-hand side of (6.25) because $\boldsymbol{e}_{\boldsymbol{u}}^{n}$ belongs to $V_{h}$.

Let us choose $\left(\boldsymbol{v}_{h}, \boldsymbol{e}_{h}\right)=\left(\boldsymbol{e}_{\boldsymbol{u}}^{n}, \boldsymbol{e}_{\boldsymbol{w}}^{n}\right) \in V_{h} \times W_{h}$ in (6.24) and substitute into it (6.25) with $\boldsymbol{g}_{h}=\mu \delta_{t} \boldsymbol{e}_{\boldsymbol{d}}^{n} \in D_{0 h}$. Since

$$
k\left(\boldsymbol{e}_{\boldsymbol{w}}^{n}, \mu \delta_{t} \boldsymbol{e}_{\boldsymbol{d}}^{n}\right)=k \mu^{2}\left(\nabla \boldsymbol{e}_{\boldsymbol{d}}^{n}, \nabla \delta_{t} \boldsymbol{e}_{\boldsymbol{d}}^{n}\right)-k \widetilde{\mathcal{E}}_{h}^{n}\left(\mu \delta_{t} \boldsymbol{e}_{\boldsymbol{d}}^{n}\right),
$$

and in view of (4.8),

$$
\begin{aligned}
& c\left(\left(I_{h}\left(\boldsymbol{u}\left(t_{n-1}\right), S Z_{h} \boldsymbol{d}\left(t_{n-1}\right)\right),\left(\boldsymbol{e}_{\boldsymbol{u}}^{n}, \boldsymbol{e}_{\boldsymbol{w}}^{n}\right),\left(\boldsymbol{e}_{\boldsymbol{u}}^{n}, \boldsymbol{e}_{\boldsymbol{w}}^{n}\right)\right)=0,\right. \\
& c\left(\left(\boldsymbol{e}_{\boldsymbol{u}}^{n-1}, \boldsymbol{e}_{\boldsymbol{d}}^{n-1}\right),\left(\boldsymbol{u}_{h}^{n}, \boldsymbol{w}_{h}^{n}\right),\left(\boldsymbol{e}_{\boldsymbol{u}}^{n}, \boldsymbol{e}_{\boldsymbol{w}}^{n}\right)\right) \\
& \quad=c\left(\left(\boldsymbol{e}_{\boldsymbol{u}}^{n-1}, \boldsymbol{e}_{\boldsymbol{d}}^{n-1}\right),\left(I_{h} \boldsymbol{u}\left(t_{n}\right), K_{h} \boldsymbol{w}\left(t_{n}\right)\right),\left(\boldsymbol{e}_{\boldsymbol{u}}^{n}, \boldsymbol{e}_{\boldsymbol{w}}^{n}\right)\right),
\end{aligned}
$$

this gives

$$
\begin{aligned}
& \frac{1}{2}\left\{\left\|\boldsymbol{e}_{\boldsymbol{u}}^{n}\right\|_{\boldsymbol{L}^{2}(\Omega)}^{2}-\left\|\boldsymbol{e}_{\boldsymbol{u}}^{n-1}\right\|_{\boldsymbol{L}^{2}(\Omega)}^{2}+\left\|\boldsymbol{e}_{\boldsymbol{u}}^{n}-\boldsymbol{e}_{\boldsymbol{u}}^{n-1}\right\|_{\boldsymbol{L}^{2}(\Omega)}^{2}\right\} \\
& +\frac{\mu^{2}}{2}\left\{\left|\boldsymbol{e}_{\boldsymbol{d}}^{n}\right|_{\boldsymbol{H}^{1}(\Omega)}^{2}-\left|\boldsymbol{e}_{\boldsymbol{d}}^{n-1}\right|_{\boldsymbol{H}^{1}(\Omega)}^{2}+\left|\boldsymbol{e}_{\boldsymbol{d}}^{n}-\boldsymbol{e}_{\boldsymbol{d}}^{n-1}\right|_{\boldsymbol{H}^{1}(\Omega)}^{2}\right\} \\
& +\nu k\left|\boldsymbol{e}_{\boldsymbol{u}}^{n}\right|_{\boldsymbol{H}^{1}(\Omega)}^{2}+\gamma k\left\|\boldsymbol{e}_{\boldsymbol{w}}^{n}\right\|_{\boldsymbol{L}^{2}(\Omega)}^{2}=\mathcal{E}_{h}^{n}\left(\boldsymbol{e}_{\boldsymbol{u}}^{n}, \boldsymbol{e}_{\boldsymbol{w}}^{n}\right)+k \mu \widetilde{\mathcal{E}}_{h}^{n}\left(\delta_{t} \boldsymbol{e}_{\boldsymbol{d}}^{n}\right) \\
& -k c\left(\left(\boldsymbol{e}_{\boldsymbol{u}}^{n-1}, \boldsymbol{e}_{\boldsymbol{d}}^{n-1}\right),\left(I_{h} \boldsymbol{u}\left(t_{n}\right), K_{h} \boldsymbol{w}\left(t_{n}\right)\right),\left(\boldsymbol{e}_{\boldsymbol{u}}^{n}, \boldsymbol{e}_{\boldsymbol{w}}^{n}\right)\right) \\
& -k \mu \gamma\left(\tilde{\boldsymbol{f}}\left(\boldsymbol{d}_{h}^{n-1}\right)-\widetilde{\boldsymbol{f}}\left(S Z_{h} \boldsymbol{d}\left(t_{n-1}\right)\right), \boldsymbol{e}_{\boldsymbol{w}}^{n}\right) \\
& :=I_{1}+I_{2}+I_{3}+I_{4} .
\end{aligned}
$$

Let us bound first $I_{1}, I_{3}$ and $I_{4}$. The bound for $I_{1}$ is based on (6.13), (6.16) - 6.22). Next,

$$
\begin{aligned}
\left|I_{3}\right| \leq & C k\left\|\boldsymbol{u}\left(t_{n}\right), \boldsymbol{w}\left(t_{n}\right)\right\|_{\left(\boldsymbol{W}^{1,3}(\Omega) \cap \boldsymbol{L}^{\infty}(\Omega)\right) \times \boldsymbol{L}^{3}(\Omega)} \\
& \times\left\|\boldsymbol{e}_{\boldsymbol{u}}^{n-1}, \mu \boldsymbol{e}_{\boldsymbol{d}}^{n-1}\right\|_{\boldsymbol{L}^{2}(\Omega) \times \boldsymbol{H}^{1}(\Omega)}\left\|\boldsymbol{e}_{\boldsymbol{u}}^{n}, \boldsymbol{e}_{\boldsymbol{w}}^{n}\right\|_{X},
\end{aligned}
$$

owing to the first part of (6.7) with $p=3, n=1$ and the first part of (6.9) with $p=3$. Finally,

$$
\begin{aligned}
\left|I_{4}\right| & \leq \mu \gamma \frac{k}{\varepsilon^{2}} \int_{\Omega} \operatorname{Max}\left(3,1+\left|\boldsymbol{d}_{h}^{n-1}\right|\right)\left|\boldsymbol{e}_{\boldsymbol{d}}^{n-1} \| \boldsymbol{e}_{\boldsymbol{w}}^{n}\right| d \boldsymbol{x} \\
& \leq C k \frac{\mu \gamma}{\varepsilon^{2}}\left(\left\|\boldsymbol{d}_{h}^{n-1}\right\|_{\boldsymbol{L}^{3}(\Omega)}+1\right)\left\|\boldsymbol{e}_{\boldsymbol{d}}^{n-1}\right\|_{\boldsymbol{L}^{6}(\Omega)}\left\|\boldsymbol{e}_{\boldsymbol{w}}^{n}\right\|_{\boldsymbol{L}^{2}(\Omega)} \\
& \leq C k \frac{\mu \gamma}{\varepsilon^{2}}\left\|\boldsymbol{e}_{\boldsymbol{d}}^{n-1}\right\|_{\boldsymbol{L}^{6}(\Omega)}\left\|\boldsymbol{e}_{\boldsymbol{w}}^{n}\right\|_{\boldsymbol{L}^{2}(\Omega)}
\end{aligned}
$$

owing to properties (8.1) and (8.2) of $\tilde{f}$ established in the Appendix, and owing to the uniform bound for $\left\|\boldsymbol{d}_{h}^{n-1}\right\|_{\boldsymbol{L}^{3}(\Omega)}$ that stems from (5.15).

Accordingly, by summing (6.26) from $n=1$ to $n=m$, applying suitably Young's inequality, and using the above inequalities, estimate for $\boldsymbol{d}_{h}^{n}$ given in (5.15), and 
the fact that the initial errors are zero, we derive the intermediate error inequality:

$$
\begin{aligned}
& \left\|\boldsymbol{e}_{\boldsymbol{u}}^{m}\right\|_{\boldsymbol{L}^{2}(\Omega)}^{2}+\mu^{2}\left|\boldsymbol{e}_{\boldsymbol{d}}^{m}\right|_{\boldsymbol{H}^{1}(\Omega)}^{2}+\sum_{n=1}^{m}\left(\left\|\boldsymbol{e}_{\boldsymbol{u}}^{n}-\boldsymbol{e}_{\boldsymbol{u}}^{n-1}\right\|_{\boldsymbol{L}^{2}(\Omega)}^{2}+\mu^{2}\left|\boldsymbol{e}_{\boldsymbol{d}}^{n}-\boldsymbol{e}_{\boldsymbol{d}}^{n-1}\right|_{\boldsymbol{H}^{1}(\Omega)}^{2}\right) \\
& +\sum_{n=1}^{m} k\left(\nu\left|\boldsymbol{e}_{\boldsymbol{u}}^{n}\right|_{\boldsymbol{H}^{1}(\Omega)}^{2}+\gamma\left\|\boldsymbol{e}_{\boldsymbol{w}}^{n}\right\|_{\boldsymbol{L}^{2}(\Omega)}^{2}\right) \\
& \leq C\left(\|\boldsymbol{u}, \boldsymbol{w}\|_{L^{\infty}}^{2}\left(\left(\boldsymbol{W}^{1,3}(\Omega) \cap \boldsymbol{L}^{\infty}(\Omega)\right) \times \boldsymbol{L}^{3}(\Omega)\right)\right. \\
& +\sum_{n=1}^{m} k\left(\left\|\boldsymbol{e}_{\boldsymbol{u}}^{n-1}\right\|_{\boldsymbol{L}^{2}(\Omega)}^{2}+\mu^{2}\left|\boldsymbol{e}_{\boldsymbol{d}}^{n-1}\right|_{\boldsymbol{H}^{1}(\Omega)}^{2}\right) \\
& +C h^{2}\left(\|\boldsymbol{u}, \boldsymbol{w}\|_{L^{2}\left(\boldsymbol{H}^{2}(\Omega) \times \boldsymbol{H}^{1}(\Omega)\right)}^{2}+\|\boldsymbol{d}\|_{H^{1}\left(\boldsymbol{H}^{1}(\Omega)\right)}^{2}\right. \\
& +\left\|\boldsymbol{u}^{\prime}\right\|_{L^{2}\left(\boldsymbol{L}^{2}(\Omega)\right)}^{2}+\|p\|_{L^{2}\left(H^{1}(\Omega)\right)}^{2} \\
& +\|\boldsymbol{d}\|_{L^{\infty}\left(\boldsymbol{W}^{1,3}(\Omega)\right)}^{2}\|\boldsymbol{u}, \boldsymbol{w}\|_{L^{2}\left(\boldsymbol{W}^{1,6}(\Omega) \times \boldsymbol{H}^{1}(\Omega)\right)}^{2} \\
& +\|\boldsymbol{d}\|_{L^{2}\left(\boldsymbol{W}^{2,3}(\Omega)\right)}^{2}\|\boldsymbol{u}, \boldsymbol{w}\|_{L^{\infty}\left(\boldsymbol{H}^{1}(\Omega) \times \boldsymbol{L}^{2}(\Omega)\right)}^{2} \\
& \left.+\|\boldsymbol{u}\|_{L^{2}\left(\boldsymbol{W}^{1,3}(\Omega)\right)}^{2}\|\boldsymbol{u}\|_{L^{\infty}\left(\boldsymbol{H}^{1}(\Omega)\right)}^{2}\right) \\
& +C k^{2}\left(\left\|\boldsymbol{u}^{\prime}, \boldsymbol{w}^{\prime}\right\|_{L^{2}\left(\boldsymbol{H}^{1}(\Omega) \times \boldsymbol{L}^{2}(\Omega)\right)}^{2}+\|\boldsymbol{d}\|_{L^{\infty}\left(\boldsymbol{W}^{1,6}(\Omega)\right)}^{2}\left\|\boldsymbol{u}^{\prime}, \boldsymbol{w}^{\prime}\right\|_{L^{2}\left(\boldsymbol{L}^{3}(\Omega) \times \boldsymbol{L}^{2}(\Omega)\right)}^{2}\right. \\
& +\left\|\boldsymbol{d}^{\prime}\right\|_{L^{2}\left(\boldsymbol{H}^{1}(\Omega)\right)}^{2}+\left\|\boldsymbol{d}^{\prime}\right\|_{L^{2}\left(\boldsymbol{W}^{1,3}(\Omega)\right)}^{2}\|\boldsymbol{u}, \boldsymbol{w}\|_{L^{\infty}\left(\boldsymbol{H}^{1}(\Omega) \times \boldsymbol{L}^{2}(\Omega)\right)}^{m} \\
& \left.+\|\boldsymbol{u}\|_{L^{\infty}\left(\boldsymbol{H}^{1}(\Omega)\right)}^{2}\left\|\boldsymbol{u}^{\prime}\right\|_{L^{2}\left(\boldsymbol{L}^{3}(\Omega)\right)}^{2}\right)+2 \mu\left|\sum_{n=1}^{m} k \widetilde{\mathcal{E}}_{h}^{n}\left(\delta_{t} \boldsymbol{e}_{\boldsymbol{d}}^{n}\right)\right|
\end{aligned}
$$

In order to estimate $I_{2}$, it is useful to switch the discrete time derivative from $\boldsymbol{e}_{\boldsymbol{d}}^{n}$ to $\widetilde{\mathcal{E}}_{h}^{n}$ by means of a "discrete" integration by parts, as follows:

$$
k \mu \sum_{n=1}^{m} \widetilde{\mathcal{E}}_{h}^{n}\left(\delta_{t} \boldsymbol{e}_{\boldsymbol{d}}^{n}\right)=-k \mu \sum_{n=2}^{m} \delta_{t} \widetilde{\mathcal{E}}_{h}^{n}\left(\boldsymbol{e}_{\boldsymbol{d}}^{n-1}\right)+\mu \widetilde{\mathcal{E}}_{h}^{m}\left(\boldsymbol{e}_{\boldsymbol{d}}^{m}\right)
$$

and

$$
\begin{aligned}
\delta_{t} \widetilde{\mathcal{E}}_{h}^{m}\left(\boldsymbol{e}_{\boldsymbol{d}}^{m-1}\right)= & \mu\left(\nabla \delta_{t}\left(\boldsymbol{d}\left(t_{m}\right)-S Z_{h} \boldsymbol{d}\left(t_{m}\right)\right), \nabla \boldsymbol{e}_{d}^{m-1}\right) \\
& -\left(\delta_{t}\left(\boldsymbol{w}\left(t_{m}\right)-K_{h} \boldsymbol{w}\left(t_{m}\right)\right), \boldsymbol{e}_{d}^{m-1}\right) .
\end{aligned}
$$

Then, applying (6.23), we derive

$$
\begin{array}{rl}
2 \mid \sum_{n=1}^{m} & k \widetilde{\mathcal{E}}_{h}^{n}\left(\mu \delta_{t} \boldsymbol{e}_{\boldsymbol{d}}^{n}\right) \mid \\
& \leq C \mu h k^{1 / 2} \sum_{n=1}^{m}\left(\mu\left\|\boldsymbol{d}^{\prime}\right\|_{L^{2}\left(I_{n} ; \boldsymbol{H}^{2}(\Omega)\right)}+\left\|\boldsymbol{w}^{\prime}\right\|_{L^{2}\left(I_{n} ; \boldsymbol{W}^{1,6 / 5}(\Omega)\right)}\right)\left|\boldsymbol{e}_{\boldsymbol{d}}^{n-1}\right|_{\boldsymbol{H}^{1}(\Omega)} \\
& +C \mu h\|(\mu \boldsymbol{d}, \boldsymbol{w})\|_{L^{\infty}\left(\boldsymbol{H}^{2}(\Omega) \times \boldsymbol{W}^{1,6 / 5}(\Omega)\right)}\left|\boldsymbol{e}_{\boldsymbol{d}}^{m}\right|_{\boldsymbol{H}^{1}(\Omega)} \\
& \leq \delta \mu^{2} \sum_{n=1}^{m} k\left|\boldsymbol{e}_{\boldsymbol{d}}^{n-1}\right|_{\boldsymbol{H}^{1}(\Omega)}^{2}+\delta \mu^{2}\left|\boldsymbol{e}_{\boldsymbol{d}}^{m}\right|_{\boldsymbol{H}^{1}(\Omega)}^{2} \\
& +\frac{C}{\delta} \mu h^{2}\left(\left\|\mu \boldsymbol{d}^{\prime}, \boldsymbol{w}^{\prime}\right\|_{L^{2}\left(\boldsymbol{H}^{2}(\Omega) \times \boldsymbol{W}^{1,6 / 5}(\Omega)\right)}^{2}+\|\mu \boldsymbol{d}, \boldsymbol{w}\|_{L^{\infty}\left(\boldsymbol{H}^{2}(\Omega) \times \boldsymbol{W}^{1,6 / 5}(\Omega)\right)}^{2}\right),
\end{array}
$$


for any $\delta>0$, for instance, $\delta=1 / 2$. When substituted into (6.29), this choice yields

$$
\begin{aligned}
& \left\|\boldsymbol{e}_{\boldsymbol{u}}^{m}\right\|_{\boldsymbol{L}^{2}(\Omega)}^{2}+\frac{1}{2} \mu^{2}\left|\boldsymbol{e}_{\boldsymbol{d}}^{m}\right|_{\boldsymbol{H}^{1}(\Omega)}^{2}+\sum_{n=1}^{m}\left(\left\|\boldsymbol{e}_{\boldsymbol{u}}^{n}-\boldsymbol{e}_{\boldsymbol{u}}^{n-1}\right\|_{\boldsymbol{L}^{2}(\Omega)}^{2}+\mu^{2}\left|\boldsymbol{e}_{\boldsymbol{d}}^{n}-\boldsymbol{e}_{\boldsymbol{d}}^{n-1}\right|_{\boldsymbol{H}^{1}(\Omega)}^{2}\right) \\
& +\sum_{n=1}^{m} k\left(\nu\left|\boldsymbol{e}_{\boldsymbol{u}}^{n}\right|_{\boldsymbol{H}^{1}(\Omega)}^{2}+\gamma\left\|\boldsymbol{e}_{\boldsymbol{w}}^{n}\right\|_{\boldsymbol{L}^{2}(\Omega)}^{2}\right) \\
& \leq C\left(h^{2}+k^{2}\right)+C \sum_{n=1}^{m} k\left(\left\|\boldsymbol{e}_{\boldsymbol{u}}^{n-1}\right\|_{\boldsymbol{L}^{2}(\Omega)}^{2}+\mu^{2}\left|\boldsymbol{e}_{\boldsymbol{d}}^{n-1}\right|_{\boldsymbol{H}^{1}(\Omega)}^{2}\right) .
\end{aligned}
$$

Then Gronwall's Lemma implies the main result of this section.

Theorem 6.1. Assume that the triangulation is quasi-uniform (see (5.16)) and the solution $(\boldsymbol{u}, p, \boldsymbol{d})$ has the following regularity:

$$
\begin{gathered}
(\boldsymbol{u}, \boldsymbol{d}, \boldsymbol{w}) \in L^{2}\left(\boldsymbol{H}^{2}(\Omega) \times \boldsymbol{W}^{2,3}(\Omega) \times \boldsymbol{H}^{1}(\Omega)\right), \\
\boldsymbol{u} \in L^{\infty}\left(\boldsymbol{W}^{1,3}(\Omega) \cap \boldsymbol{L}^{\infty}(\Omega)\right), p \in L^{2}\left(H^{1}(\Omega)\right), \\
\left(\partial_{t} \boldsymbol{u}, \partial_{t} \boldsymbol{d}, \partial_{t} \boldsymbol{w}\right) \in L^{2}\left(\boldsymbol{H}^{1}(\Omega) \times \boldsymbol{H}^{2}(\Omega) \times \boldsymbol{W}^{1,6 / 5}(\Omega)\right) .
\end{gathered}
$$

Then the fully discrete scheme (5.10), (5.11) satisfies the optimal error estimates for $1 \leq m \leq n$ :

$$
\begin{aligned}
\left\|\boldsymbol{e}_{\boldsymbol{u}}^{m}\right\|_{\boldsymbol{L}^{2}(\Omega)}^{2}+\frac{1}{2} \mu^{2}\left|\boldsymbol{e}_{\boldsymbol{d}}^{m}\right|_{\boldsymbol{H}^{1}(\Omega)}^{2} & \\
+ & \sum_{n=1}^{m}\left(\left\|\boldsymbol{e}_{\boldsymbol{u}}^{n}-\boldsymbol{e}_{\boldsymbol{u}}^{n-1}\right\|_{\boldsymbol{L}^{2}(\Omega)}^{2}+\mu^{2}\left|\boldsymbol{e}_{\boldsymbol{d}}^{n}-\boldsymbol{e}_{\boldsymbol{d}}^{n-1}\right|_{\boldsymbol{H}^{1}(\Omega)}^{2}\right) \\
& +\sum_{n=1}^{m} k\left(\nu\left|\boldsymbol{e}_{\boldsymbol{u}}^{n}\right|_{\boldsymbol{H}^{1}(\Omega)}^{2}+\gamma\left\|\boldsymbol{e}_{\boldsymbol{w}}^{n}\right\|_{\boldsymbol{L}^{2}(\Omega)}^{2}\right) \leq C\left(h^{2}+k^{2}\right),
\end{aligned}
$$

with a constant $C$ that depends exponentially on $\frac{1}{\varepsilon^{2}}$, but is independent of $h, k$, and $m$.

Note that the regularity assumptions on the velocity and pressure do not require a global compatibility condition on the initial data. More precisely, Lin and Liu establish in 24] local existence in time of solutions with this regularity and global existence in time with the same regularity when the viscosity parameter $\nu$ is large enough.

Pressure error estimates are similar to those obtained for the Navier-Stokes equations 37, but as expected, they rely on upper bounds for the difference quotients $\delta_{t} \boldsymbol{e}_{\boldsymbol{u}}^{n}$ and $\delta_{t} \boldsymbol{e}_{\boldsymbol{d}}^{n}$. In three dimensions, these can be respectively derived by testing the velocity's error system with the discrete time derivative $\delta_{t} \boldsymbol{e}_{\boldsymbol{u}}^{n}$ and applying strong estimates for the linear discrete Stokes problem, and by testing the auxiliary variable's $(\boldsymbol{w})$ error system with the discrete time derivative $\delta_{t} \boldsymbol{e}_{\boldsymbol{w}}^{n}$ and applying strong estimates for the linear discrete Poisson problem. This argument is rather different from that used in deriving error estimates for the velocity and will be the subject of a forthcoming work 20 . 


\section{Decoupling the Computation of the Velocity and DiRections fields}

Computing the solution of (5.10), (5.11) is highly time-consuming because this system, albeit linear, couples all unknowns and its matrix has a complex structure. In this section, we propose three algorithms for decoupling, at least partly, this computation.

7.1. Algorithms. For any $n \geq 1$, given $\boldsymbol{w}_{h}^{n-1}, \boldsymbol{d}_{h}^{n-1}, \boldsymbol{u}_{h}^{n-1}$ and $p_{h}^{n-1}$, we present three iterative algorithms for approximating $\boldsymbol{w}_{h}^{n}, \boldsymbol{d}_{h}^{n}, \boldsymbol{u}_{h}^{n}$ and $p_{h}^{n}$.

(1) (a) Let $\boldsymbol{w}_{0}$ be a first guess for $\boldsymbol{w}_{h}^{n}$; for instance, $\boldsymbol{w}_{0}=\boldsymbol{w}_{h}^{n-1}$, if $n>1$, or $\boldsymbol{w}_{0}=-\mu K_{h}\left(\Delta \boldsymbol{d}_{0}\right)$, if $n=1$; see (6.6).

(b) For $i \geq 1$, knowing $\boldsymbol{w}_{i-1}$, compute $\left(\boldsymbol{u}_{i}, p_{i}\right) \in U_{0 h} \times P_{h}$ the solution of:

$$
\begin{aligned}
\forall \boldsymbol{v} \in U_{0 h}, \frac{1}{k}\left(\boldsymbol{u}_{i}-\boldsymbol{u}_{h}^{n-1}, \boldsymbol{v}\right)+\nu\left(\nabla \boldsymbol{u}_{i}, \nabla \boldsymbol{v}\right)-\left(p_{i}, \nabla \cdot \boldsymbol{v}\right) & \\
+\left(\boldsymbol{u}_{h}^{n-1} \cdot \nabla \boldsymbol{u}_{i}+\frac{1}{2} \nabla \cdot \boldsymbol{u}_{h}^{n-1} \boldsymbol{u}_{i}, \boldsymbol{v}\right) & =\mu\left(\left(\nabla \boldsymbol{d}_{h}^{n-1}\right)^{t} \boldsymbol{w}_{i-1}, \boldsymbol{v}\right), \\
\forall q \in P_{h},\left(\nabla \cdot \boldsymbol{u}_{i}, q\right) & =0 .
\end{aligned}
$$

(c) Next, compute $\left(\boldsymbol{w}_{i}, \boldsymbol{d}_{i}\right) \in W_{h} \times D_{h}$, with $\left.\left(\boldsymbol{d}_{i}\right)\right|_{\partial \Omega}=\boldsymbol{l}_{h}^{n}$, the solution of:

$$
\begin{aligned}
\forall \boldsymbol{e} \in W_{h}, \frac{1}{k}\left(\boldsymbol{d}_{i}-\boldsymbol{d}_{h}^{n-1}, \boldsymbol{e}\right)+\frac{\gamma}{\mu}\left(\boldsymbol{w}_{i}, \boldsymbol{e}\right)=-\left(\boldsymbol{u}_{i} \cdot \nabla \boldsymbol{d}_{h}^{n-1}, \boldsymbol{e}\right)-\gamma\left(\tilde{\boldsymbol{f}}\left(\boldsymbol{d}_{h}^{n-1}\right), \boldsymbol{e}\right) \\
\forall \boldsymbol{g} \in D_{0 h}, \mu\left(\nabla \boldsymbol{d}_{i}, \nabla \boldsymbol{g}\right)-\left(\boldsymbol{w}_{i}, \boldsymbol{g}\right)=0
\end{aligned}
$$

(2) (a) Let $\boldsymbol{u}_{0}=\boldsymbol{u}_{h}^{n-1}$.

(b) Knowing $\boldsymbol{u}_{i-1}$, compute $\left(\boldsymbol{w}_{i}, \boldsymbol{d}_{i}\right) \in W_{h} \times D_{h}$, with $\left.\left(\boldsymbol{d}_{i}\right)\right|_{\partial \Omega}=\boldsymbol{l}_{h}^{n}$, the solution of:

$$
\begin{gathered}
\forall \boldsymbol{e} \in W_{h}, \frac{1}{k}\left(\boldsymbol{d}_{i}-\boldsymbol{d}_{h}^{n-1}, \boldsymbol{e}\right)+\frac{\gamma}{\mu}\left(\boldsymbol{w}_{i}, \boldsymbol{e}\right)=-\left(\boldsymbol{u}_{i-1} \cdot \nabla \boldsymbol{d}_{h}^{n-1}, \boldsymbol{e}\right)-\gamma\left(\tilde{\boldsymbol{f}}\left(\boldsymbol{d}_{h}^{n-1}\right), \boldsymbol{e}\right), \\
\forall \boldsymbol{g} \in D_{0 h}, \mu\left(\nabla \boldsymbol{d}_{i}, \nabla \boldsymbol{g}\right)-\left(\boldsymbol{w}_{i}, \boldsymbol{g}\right)=0 .
\end{gathered}
$$

(c) Next, compute $\left(\boldsymbol{u}_{i}, p_{i}\right) \in U_{0 h} \times P_{h}$ solving:

$$
\begin{aligned}
\forall \boldsymbol{v} \in U_{0 h}, \frac{1}{k}\left(\boldsymbol{u}_{i}-\boldsymbol{u}_{h}^{n-1}, \boldsymbol{v}\right)+\nu\left(\nabla \boldsymbol{u}_{i}, \nabla \boldsymbol{v}\right)-\left(p_{i}, \nabla \cdot \boldsymbol{v}\right) & \\
+\left(\boldsymbol{u}_{h}^{n-1} \cdot \nabla \boldsymbol{u}_{i}+\frac{1}{2} \nabla \cdot \boldsymbol{u}_{h}^{n-1} \boldsymbol{u}_{i}, \boldsymbol{v}\right) & =\mu\left(\left(\nabla \boldsymbol{d}_{h}^{n-1}\right)^{t} \boldsymbol{w}_{i}, \boldsymbol{v}\right), \\
\forall q \in P_{h},\left(\nabla \cdot \boldsymbol{u}_{i}, q\right) & =0 .
\end{aligned}
$$

(3) (a) Let $\boldsymbol{u}_{0}=\boldsymbol{u}_{h}^{n-1}$ and $\boldsymbol{w}_{0}=\boldsymbol{w}_{h}^{n-1}$, if $n>1$, or $\boldsymbol{w}_{0}=-\mu K_{h}\left(\Delta \boldsymbol{d}_{0}\right)$, if $n=1$.

(b) Knowing $\boldsymbol{u}_{i-1}$ and $\boldsymbol{w}_{i-1}$, compute in parallel

- $\left(\boldsymbol{w}_{i}, \boldsymbol{d}_{i}\right) \in W_{h} \times D_{h}$, with $\left.\left(\boldsymbol{d}_{i}\right)\right|_{\partial \Omega}=\boldsymbol{l}_{h}^{n}$, the solution of

$$
\begin{gathered}
\forall \boldsymbol{e} \in W_{h}, \frac{1}{k}\left(\boldsymbol{d}_{i}-\boldsymbol{d}_{h}^{n-1}, \boldsymbol{e}\right)+\frac{\gamma}{\mu}\left(\boldsymbol{w}_{i}, \boldsymbol{e}\right)=-\left(\boldsymbol{u}_{i-1} \cdot \nabla \boldsymbol{d}_{h}^{n-1}, \boldsymbol{e}\right)-\gamma\left(\tilde{\boldsymbol{f}}\left(\boldsymbol{d}_{h}^{n-1}\right), \boldsymbol{e}\right) \\
\forall \boldsymbol{g} \in D_{0 h}, \mu\left(\nabla \boldsymbol{d}_{i}, \nabla \boldsymbol{g}\right)-\left(\boldsymbol{w}_{i}, \boldsymbol{g}\right)=0
\end{gathered}
$$


- and $\left(\boldsymbol{u}_{i}, p_{i}\right) \in U_{0 h} \times P_{h}$, the solution of

$$
\begin{aligned}
\forall \boldsymbol{v} & \in U_{0 h}, \frac{1}{k}\left(\boldsymbol{u}_{i}-\boldsymbol{u}_{h}^{n-1}, \boldsymbol{v}\right)+\nu\left(\nabla \boldsymbol{u}_{i}, \nabla \boldsymbol{v}\right)-\left(p_{i}, \nabla \cdot \boldsymbol{v}\right) \\
& =\mu\left(\left(\nabla \boldsymbol{d}_{h}^{n-1}\right)^{t} \boldsymbol{w}_{i-1}, \boldsymbol{v}\right)-\left(\boldsymbol{u}_{h}^{n-1} \cdot \nabla \boldsymbol{u}_{i-1}+\frac{1}{2} \nabla \cdot \boldsymbol{u}_{h}^{n-1} \boldsymbol{u}_{i-1}, \boldsymbol{v}\right),
\end{aligned}
$$$$
\forall q \in P_{h},\left(\nabla \cdot \boldsymbol{u}_{i}, q\right)=0 .
$$

In the practical implementation of these three schemes, the problems for $\left(\boldsymbol{w}_{i}, \boldsymbol{d}_{i}\right)$ have the generic form: Given $\boldsymbol{F}$, compute $(\boldsymbol{w}, \boldsymbol{d}) \in W_{h} \times D_{h}$, with $\left.\boldsymbol{d}\right|_{\partial \Omega}=\boldsymbol{l}_{h}^{n}$, such that:

$$
\begin{aligned}
\forall \boldsymbol{e} \in W_{h}, \mu \frac{1}{k}(\boldsymbol{d}, \boldsymbol{e})+\gamma(\boldsymbol{w}, \boldsymbol{e}) & =(\boldsymbol{F}, \boldsymbol{e}), \\
\forall \boldsymbol{g} \in D_{0 h}, \mu(\nabla \boldsymbol{d}, \nabla \boldsymbol{g})-(\boldsymbol{w}, \boldsymbol{g}) & =0 .
\end{aligned}
$$

Note that, since $W_{h}$ is generated by constant functions in each element, the term $\gamma(\boldsymbol{w}, \boldsymbol{e})$ has a diagonal mass matrix, and hence (7.1) defines $\boldsymbol{w}$ as an explicit function of $\boldsymbol{d}$. When substituted into (17.2), this gives a linear elliptic equation where $\boldsymbol{d}$ is the only unknown. Once this equation is solved, $\boldsymbol{w}$ is recovered from its explicit expression in terms of $\boldsymbol{d}$.

Remark 7.1. If we change the discrete space $W_{h}$ to the space generated by $\mathbb{P}_{1}$ finite elements, i.e., $W_{h}=D_{h}$, then choosing $\boldsymbol{e}=\boldsymbol{g} \in D_{0 h}$ in (7.1) and using (7.2), we arrive at the following problem: Compute $\boldsymbol{d} \in D_{h}$ with $\left.\boldsymbol{d}\right|_{\partial \Omega}=\boldsymbol{l}_{h}^{n}$ such that

$$
\forall \boldsymbol{g} \in D_{0 h}, \frac{1}{k}(\boldsymbol{d}, \boldsymbol{g})+\gamma(\nabla \boldsymbol{d}, \nabla \boldsymbol{g})=\frac{1}{\mu}(\boldsymbol{F}, \boldsymbol{g}) .
$$

Afterward, $\boldsymbol{w}$ can be computed from (7.1), the matrix of this system being the mass matrix (with $\mathbb{P}_{1}$ basis functions) arising from the term $\gamma(\boldsymbol{w}, \boldsymbol{e})$.

7.2. Convergence. The convergence of these algorithms when $i \rightarrow \infty$ (for fixed $h, k, n)$ follows from a fixed-point argument. For the sake of conciseness, we only present this proof for the parallel algorithm that achieves a stronger decoupling, but the proofs of convergence for the other algorithms follow the same lines.

Introducing the notation

$$
\boldsymbol{U}_{i}=\boldsymbol{u}_{i}-\boldsymbol{u}_{i-1}, \quad P_{i}=p_{i}-p_{i-1}, \quad \boldsymbol{D}_{i}=\boldsymbol{d}_{i}-\boldsymbol{d}_{i-1}, \quad \boldsymbol{W}_{i}=\boldsymbol{w}_{i}-\boldsymbol{w}_{i-1},
$$

we obtain the following equations for $i \geq 2$ :

- $\left(\boldsymbol{W}_{i}, \boldsymbol{D}_{i}\right) \in W_{h} \times D_{0 h}$, satisfies:

$$
\begin{aligned}
\forall \boldsymbol{e} \in W_{h}, \frac{\mu}{k}\left(\boldsymbol{D}_{i}, \boldsymbol{e}\right)+\gamma\left(\boldsymbol{W}_{i}, \boldsymbol{e}\right) & =-\mu\left(\boldsymbol{U}_{i-1} \cdot \nabla \boldsymbol{d}_{h}^{n-1}, \boldsymbol{e}\right), \\
\forall \boldsymbol{g} \in D_{0 h}, \mu\left(\nabla \boldsymbol{D}_{i}, \nabla \boldsymbol{g}\right) & =\left(\boldsymbol{W}_{i}, \boldsymbol{g}\right)
\end{aligned}
$$

- $\left(\boldsymbol{U}_{i}, P_{i}\right) \in U_{0 h} \times P_{h}$ satisfies:

$$
\begin{aligned}
\forall \boldsymbol{v} \in U_{0 h}, \frac{1}{k}\left(\boldsymbol{U}_{i}, \boldsymbol{v}\right)+\nu & \left(\nabla \boldsymbol{U}_{i}, \nabla \boldsymbol{v}\right)-\left(P_{i}, \nabla \cdot \boldsymbol{v}\right)=\mu\left(\left(\nabla \boldsymbol{d}_{h}^{n-1}\right)^{t} \boldsymbol{W}_{i-1}, \boldsymbol{v}\right) \\
& -\left(\boldsymbol{u}_{h}^{n-1} \cdot \nabla \boldsymbol{U}_{i-1}+\frac{1}{2} \nabla \cdot \boldsymbol{u}_{h}^{n-1} \boldsymbol{U}_{i-1}, \boldsymbol{v}\right) \\
\forall q \in P_{h},\left(\nabla \cdot \boldsymbol{U}_{i}, q\right) & =0 .
\end{aligned}
$$


The first system with test function $(\boldsymbol{e}, \boldsymbol{g})=\left(\boldsymbol{W}_{i}, \boldsymbol{D}_{i}\right)$ gives

$$
\frac{1}{k}\left\|\mu \nabla \boldsymbol{D}_{i}\right\|_{\boldsymbol{L}^{2}(\Omega)}^{2}+\gamma\left\|\boldsymbol{W}_{i}\right\|_{\boldsymbol{L}^{2}(\Omega)}^{2}=-\mu\left(\boldsymbol{U}_{i-1} \cdot \nabla \boldsymbol{d}_{h}^{n-1}, \boldsymbol{W}_{i}\right) .
$$

The second system with test function $(\boldsymbol{v}, q)=\left(\boldsymbol{U}_{i}, P_{i}\right)$ becomes

$$
\begin{gathered}
\frac{1}{k}\left\|\boldsymbol{U}_{i}\right\|_{\boldsymbol{L}^{2}(\Omega)}^{2}+\nu\left\|\nabla \boldsymbol{U}_{i}\right\|_{\boldsymbol{L}^{2}(\Omega)}^{2}=\mu\left(\left(\nabla \boldsymbol{d}_{h}^{n-1}\right)^{t} \boldsymbol{W}_{i-1}, \boldsymbol{U}_{i}\right) \\
-\left(\boldsymbol{u}_{h}^{n-1} \cdot \nabla \boldsymbol{U}_{i-1}+\frac{1}{2} \nabla \cdot \boldsymbol{u}_{h}^{n-1} \boldsymbol{U}_{i-1}, \boldsymbol{U}_{i}\right) .
\end{gathered}
$$

By adding these two equations, we have the following a priori estimates for $\boldsymbol{W}_{i}, \boldsymbol{D}_{i}$ and $\boldsymbol{U}_{i}$ :

$$
\begin{aligned}
& \frac{1}{k}\left(\left\|\mu \nabla \boldsymbol{D}_{i}\right\|_{\boldsymbol{L}^{2}(\Omega)}^{2}+\left\|\boldsymbol{U}_{i}\right\|_{\boldsymbol{L}^{2}(\Omega)}^{2}\right)+\gamma\left\|\boldsymbol{W}_{i}\right\|_{\boldsymbol{L}^{2}(\Omega)}^{2}+\nu\left\|\nabla \boldsymbol{U}_{i}\right\|_{\boldsymbol{L}^{2}(\Omega)}^{2} \\
& \quad \leq\left\|\boldsymbol{u}_{h}^{n-1}\right\|_{\boldsymbol{L}^{6}(\Omega)}\left\|\nabla \boldsymbol{U}_{i-1}\right\|_{\boldsymbol{L}^{2}(\Omega)}\left\|\boldsymbol{U}_{i}\right\|_{\boldsymbol{L}^{3}(\Omega)} \\
& \quad+\frac{1}{2}\left\|\nabla \cdot \boldsymbol{u}_{h}^{n-1}\right\|_{\boldsymbol{L}^{2}(\Omega)}\left\|\boldsymbol{U}_{i-1}\right\|_{\boldsymbol{L}^{6}(\Omega)}\left\|\boldsymbol{U}_{i}\right\|_{\boldsymbol{L}^{3}(\Omega)} \\
& \quad+\mu\left\|\nabla \boldsymbol{d}_{h}^{n-1}\right\|_{\boldsymbol{L}^{6}(\Omega)}\left(\left\|\boldsymbol{W}_{i-1}\right\|_{\boldsymbol{L}^{2}(\Omega)}\left\|\boldsymbol{U}_{i}\right\|_{\boldsymbol{L}^{3}(\Omega)}+\left\|\boldsymbol{U}_{i-1}\right\|_{\boldsymbol{L}^{3}(\Omega)}\left\|\boldsymbol{W}_{i}\right\|_{\boldsymbol{L}^{2}(\Omega)}\right)
\end{aligned}
$$

These estimates yield useful bounds provided $\boldsymbol{d}_{h}^{n}$ is bounded in $\boldsymbol{W}^{1,6}(\Omega)$ and $\boldsymbol{u}_{h}^{n}$ is bounded in $\boldsymbol{H}^{1}(\Omega)$. This is the subject of the next lemma.

Lemma 7.2. Let the space and time steps satisfy the mild condition $h^{2} \leq \theta k$ for some constant $\theta$ independent of $h$ and $k$. Suppose $\Omega$ is convex and $\boldsymbol{l}$ is the trace of a function $\boldsymbol{l}$ in $\boldsymbol{H}^{2}(\Omega)$. Then, under the hypotheses of Theorem 6.1 , there exists a constant $C$, independent of $h$ and $n$, such that

$$
\forall n \geq 1,\left\|\boldsymbol{d}_{h}^{n}\right\|_{\boldsymbol{W}^{1,6}(\Omega)} \leq C .
$$

Similarly,

$$
\forall n \geq 1,\left\|\boldsymbol{u}_{h}^{n}\right\|_{\boldsymbol{H}^{1}(\Omega)} \leq C .
$$

Proof. The proof is a straightforward variant of that of Lemma 5.5, with the function $\boldsymbol{d}(h)$ defined by

$$
-\mu \Delta \boldsymbol{d}(h)=\boldsymbol{w}_{h}^{n} \quad \text { in } \Omega,\left.\quad \boldsymbol{d}(h)\right|_{\partial \Omega}=\left.\boldsymbol{L}\right|_{\partial \Omega} .
$$

The function $\boldsymbol{d}(h)-\boldsymbol{L} \in \boldsymbol{H}_{0}^{1}(\Omega)$ solves a Laplace equation with right-hand side $\boldsymbol{w}_{h}^{n}+\mu \Delta \boldsymbol{L} \in \boldsymbol{L}^{2}(\Omega)$. Since $\Omega$ is convex, it belongs to $\boldsymbol{H}^{2}(\Omega)$ and we easily derive that

$$
\|\boldsymbol{d}(h)\|_{\boldsymbol{W}^{1,6}(\Omega)} \leq C\left(\left\|\boldsymbol{w}_{h}^{n}\right\|_{\boldsymbol{L}^{2}(\Omega)}+\mu\|\boldsymbol{L}\|_{\boldsymbol{H}^{2}(\Omega)}\right) .
$$

Then (7.4) follows from the a priori estimate (6.30), and the fact that the relation between $h$ and $k$ implies that there exists a constant $C$, independent of $h$ and $n$, such that

$$
\forall n \geq 1,\left\|\boldsymbol{w}_{h}^{n}\right\|_{\boldsymbol{L}^{2}(\Omega)} \leq C .
$$

The second inequality (7.5) is a straightforward consequence of (6.30) and the relation between $h$ and $k$.

Then, we have the following convergence theorem. 
Theorem 7.3. Under the hypotheses of Lemma 7.2, and if $k$ is sufficiently small, there exists a constant $L<1$, independent of $h$ and $k$, such that for all $i \geq 1$ :

$$
\begin{aligned}
& \left\|\mu \nabla\left(\boldsymbol{d}_{i}-\boldsymbol{d}_{h}^{n}\right)\right\|_{\boldsymbol{L}^{2}(\Omega)}^{2}+\left\|\boldsymbol{u}_{i}-\boldsymbol{u}_{h}^{n}\right\|_{\boldsymbol{L}^{2}(\Omega)}^{2}+k\left(\gamma\left\|\boldsymbol{w}_{i}-\boldsymbol{w}_{h}^{n}\right\|_{\boldsymbol{L}^{2}(\Omega)}^{2}\right. \\
& \left.\quad+\nu\left\|\nabla\left(\boldsymbol{u}_{i}-\boldsymbol{u}_{h}^{n}\right)\right\|_{\boldsymbol{L}^{2}(\Omega)}^{2}\right) \\
& \leq L^{i}\left\{\left\|\boldsymbol{u}_{h}^{n-1}-\boldsymbol{u}_{h}^{n}\right\|_{\boldsymbol{L}^{2}(\Omega)}^{2}+k\left(\gamma\left\|\boldsymbol{w}_{h}^{n-1}-\boldsymbol{w}_{h}^{n}\right\|_{\boldsymbol{L}^{2}(\Omega)}^{2}+\nu\left\|\nabla\left(\boldsymbol{u}_{h}^{n-1}-\boldsymbol{u}_{h}^{n}\right)\right\|_{\boldsymbol{L}^{2}(\Omega)}^{2}\right)\right\} .
\end{aligned}
$$

Proof. For any $i \geq 1$, set

$$
A_{i}=\left\|\mu \nabla \boldsymbol{D}_{i}\right\|_{\boldsymbol{L}^{2}(\Omega)}^{2}+\left\|\boldsymbol{U}_{i}\right\|_{\boldsymbol{L}^{2}(\Omega)}^{2}+k\left(\gamma\left\|\boldsymbol{W}_{i}\right\|_{\boldsymbol{L}^{2}(\Omega)}^{2}+\nu\left\|\nabla \boldsymbol{U}_{i}\right\|_{\boldsymbol{L}^{2}(\Omega)}^{2}\right) .
$$

By substituting (7.4) and (7.5) into (7.3), and applying the interpolation inequality

$$
\forall v \in H_{0}^{1}(\Omega),\|v\|_{L^{3}(\Omega)}^{2} \leq C\|v\|_{L^{2}(\Omega)}\|\nabla v\|_{L^{2}(\Omega)},
$$

and Young's inequality, we obtain

$$
\begin{aligned}
A_{i} \leq & C k\left(\left\|\nabla \boldsymbol{U}_{i-1}\right\|_{\boldsymbol{L}^{2}(\Omega)}\left\|\boldsymbol{U}_{i}\right\|_{\boldsymbol{L}^{2}(\Omega)}^{1 / 2}\left\|\nabla \boldsymbol{U}_{i}\right\|_{\boldsymbol{L}^{2}(\Omega)}^{1 / 2}\right. \\
& +\mu\left\|\boldsymbol{W}_{i-1}\right\|_{\boldsymbol{L}^{2}(\Omega)}\left\|\boldsymbol{U}_{i}\right\|_{\boldsymbol{L}^{2}(\Omega)}^{1 / 2}\left\|\nabla \boldsymbol{U}_{i}\right\|_{\boldsymbol{L}^{2}(\Omega)}^{1 / 2} \\
& \left.+\mu\left\|\boldsymbol{W}_{i}\right\|_{\boldsymbol{L}^{2}(\Omega)}\left\|\boldsymbol{U}_{i-1}\right\|_{\boldsymbol{L}^{2}(\Omega)}^{1 / 2}\left\|\nabla \boldsymbol{U}_{i-1}\right\|_{\boldsymbol{L}^{2}(\Omega)}^{1 / 2}\right) \\
\leq & \frac{1}{2} A_{i}+\frac{1}{2} E \sqrt{k}\left(\left\|\boldsymbol{U}_{i-1}\right\|_{\boldsymbol{L}^{2}(\Omega)}^{2}+k\left(\gamma\left\|\boldsymbol{W}_{i-1}\right\|_{\boldsymbol{L}^{2}(\Omega)}^{2}+\nu\left\|\nabla \boldsymbol{U}_{i-1}\right\|_{\boldsymbol{L}^{2}(\Omega)}^{2}\right)\right),
\end{aligned}
$$

where $E=E(\mu, \gamma, \nu)>0$. Let $0<L<1$ be a fixed number and define $k_{0}>0$ by

$$
\sqrt{k_{0}}=\frac{L}{E} \quad\left(\text { i.e. } E \sqrt{k_{0}}=L\right) .
$$

Then, for all $k \leq k_{0}$, we have

$$
A_{i} \leq L A_{i-1}
$$

Thus the mapping defining $\boldsymbol{d}_{i}, \boldsymbol{u}_{i}, \boldsymbol{w}_{i}$ is a contraction and

$$
\lim _{i \rightarrow \infty} \boldsymbol{d}_{i}=\boldsymbol{d}_{h}^{n} \text { in } \boldsymbol{H}^{1}(\Omega), \lim _{i \rightarrow \infty} \boldsymbol{u}_{i}=\boldsymbol{u}_{h}^{n} \text { in } \boldsymbol{H}^{1}(\Omega), \lim _{i \rightarrow \infty} \boldsymbol{w}_{i}=\boldsymbol{w}_{h}^{n} \text { in } \boldsymbol{L}^{2}(\Omega) .
$$

Of course, by taking the difference between the equations of the algorithm and (5.10), (5.11), we immediately derive that the differences $\boldsymbol{u}_{i}-\boldsymbol{u}_{h}^{n}, \boldsymbol{d}_{i}-\boldsymbol{d}_{h}^{n}, \boldsymbol{w}_{i}-\boldsymbol{w}_{h}^{n}$ satisfy the analogue of the estimate (17.3) with the same constants. Therefore, the above argument yields

$$
\begin{aligned}
\| \mu & \nabla\left(\boldsymbol{d}_{i}-\boldsymbol{d}_{h}^{n}\right)\left\|_{\boldsymbol{L}^{2}(\Omega)}^{2}+\right\| \boldsymbol{u}_{i}-\boldsymbol{u}_{h}^{n} \|_{\boldsymbol{L}^{2}(\Omega)}^{2} \\
& +k\left(\gamma\left\|\boldsymbol{w}_{i}-\boldsymbol{w}_{h}^{n}\right\|_{\boldsymbol{L}^{2}(\Omega)}^{2}+\nu\left\|\nabla\left(\boldsymbol{u}_{i}-\boldsymbol{u}_{h}^{n}\right)\right\|_{\boldsymbol{L}^{2}(\Omega)}^{2}\right) \\
\leq & L^{i}\left\{\left\|\boldsymbol{u}_{0}-\boldsymbol{u}_{h}^{n}\right\|_{\boldsymbol{L}^{2}(\Omega)}^{2}+k\left(\gamma\left\|\boldsymbol{w}_{0}-\boldsymbol{w}_{h}^{n}\right\|_{\boldsymbol{L}^{2}(\Omega)}^{2}+\nu\left\|\nabla\left(\boldsymbol{u}_{0}-\boldsymbol{u}_{h}^{n}\right)\right\|_{\boldsymbol{L}^{2}(\Omega)}^{2}\right)\right\},
\end{aligned}
$$

thus proving (7.6). 


\section{APPENDIX}

Recall the definition of $\tilde{\boldsymbol{f}}$ :

$$
\forall \boldsymbol{d} \in \mathbb{R}^{N}, \widetilde{\boldsymbol{f}}(\boldsymbol{d})= \begin{cases}\frac{1}{\varepsilon^{2}}\left(|\boldsymbol{d}|^{2}-1\right) \boldsymbol{d} & \text { if }|\boldsymbol{d}| \leq 1, \\ \mathbf{0} & \text { otherwise. }\end{cases}
$$

Proposition 8.1. The function $\widetilde{\boldsymbol{f}}$ is locally Lipschitz-continuous in the following sense:

(i) For all $\boldsymbol{d}, \boldsymbol{e} \in \mathbb{R}^{N}$ such that $|\boldsymbol{d}| \leq 1$ and $|\boldsymbol{e}| \leq 1$, we have

$$
|\widetilde{\boldsymbol{f}}(\boldsymbol{d})-\tilde{\boldsymbol{f}}(\boldsymbol{e})| \leq \frac{3}{\varepsilon^{2}}|\boldsymbol{d}-\boldsymbol{e}| .
$$

(ii) For all $\boldsymbol{d}, \boldsymbol{e} \in \mathbb{R}^{N}$ such that $|\boldsymbol{d}|>1$ and $|\boldsymbol{e}| \leq 1$, we have

$$
|\widetilde{\boldsymbol{f}}(\boldsymbol{d})-\widetilde{\boldsymbol{f}}(\boldsymbol{e})| \leq \frac{1}{\varepsilon^{2}}(1+|\boldsymbol{d}|)|\boldsymbol{d}-\boldsymbol{e}| .
$$

Proof. Let $|\boldsymbol{d}| \leq 1$ and $|\boldsymbol{e}| \leq 1$; then

$$
\begin{aligned}
\widetilde{\boldsymbol{f}}(\boldsymbol{d})-\widetilde{\boldsymbol{f}}(\boldsymbol{e}) & =\frac{1}{\varepsilon^{2}}\left\{\left(|\boldsymbol{d}|^{2}-1\right) \boldsymbol{d}-\left(|\boldsymbol{e}|^{2}-1\right) \boldsymbol{e}\right\} \\
& =\frac{1}{\varepsilon^{2}}\left\{\left(|\boldsymbol{d}|^{2}-1\right)(\boldsymbol{d}-\boldsymbol{e})+\left(|\boldsymbol{d}|^{2}-|\boldsymbol{e}|^{2}\right) \boldsymbol{e}\right\} \\
& =\frac{1}{\varepsilon^{2}}\left\{\left(|\boldsymbol{d}|^{2}-1\right)(\boldsymbol{d}-\boldsymbol{e})+(\boldsymbol{d}-\boldsymbol{e}) \cdot(\boldsymbol{d}+\boldsymbol{e}) \boldsymbol{e}\right\} \\
& \leq \frac{1}{\varepsilon^{2}}|\boldsymbol{d}-\boldsymbol{e}|\left\{\left.|| \boldsymbol{d}\right|^{2}-1|+| \boldsymbol{d}|+| \boldsymbol{e} \mid\right\} \\
& \leq \frac{3}{\varepsilon^{2}}|\boldsymbol{d}-\boldsymbol{e}| .
\end{aligned}
$$

Similarly, let $|\boldsymbol{e}| \leq 1$ and $|\boldsymbol{d}|>1$, then

$$
\begin{aligned}
& |\widetilde{\boldsymbol{f}}(\boldsymbol{d})-\widetilde{\boldsymbol{f}}(\boldsymbol{e})|=|\widetilde{\boldsymbol{f}}(\boldsymbol{e})|=\frac{1}{\varepsilon^{2}}\left(1-|\boldsymbol{e}|^{2}\right)|\boldsymbol{e}| \\
& \leq \frac{1}{\varepsilon^{2}}\left(|\boldsymbol{d}|^{2}-|\boldsymbol{e}|^{2}\right)=\frac{1}{\varepsilon^{2}}(\boldsymbol{d}-\boldsymbol{e}) \cdot(\boldsymbol{d}+\boldsymbol{e}) \leq \frac{1}{\varepsilon^{2}}(|\boldsymbol{d}|+1)|\boldsymbol{d}-\boldsymbol{e}| .
\end{aligned}
$$

\section{REFERENCES}

[1] Adams, R. A., Sobolev Spaces, Academic Press, New York, NY, 1975. MR 0450957 (56:9247)

[2] Arnold, D., Brezzi, F. and Fortin, M., A stable finite element for the Stokes equations, Calcolo, 21, 4 (1984), pp. 337-344. MR799997 (86m:65136)

[3] Babuška, I., The finite element method with Lagrangian multipliers, Numer. Math., 20 (1973), pp. 179-192. MR0359352 (50:11806)

[4] Becker, R., Feng, X, Prohl, A., Finite element approximations of the Ericksen-Leslie model for nematic liquid crystal flow, SIAM J. Numer. Anal. 46 4, (2008), pp. 1704-1731. MR.2399392 (2010a:65182)

[5] Bethuel, F., Brezis, H. and Hélein, F., Asymptotics for the minimization of a GinzburgLandau functional, Calc. Var., 1 (1993), pp. 123-148. MR1261720 (94m:35083)

[6] Brenner, S. and Scott, L. R., The mathematical theory of finite element methods, TAM 15, Springer-Verlag, Berlin, 1994. MR:1278258 (95f:65001)

[7] Brezzi, F., On the existence, uniqueness and approximation of saddle-point problems arising from Lagrange multipliers, RAIRO, Anal. Num., R2 (1974), pp. 129-151. MR0365287 (51:1540)

[8] Climent-Ezquerra, B., Guillén-González, F. and Rojas-Medar, M. A, Reproductivity solutions for a nematic liquid crystal model, Z. Angew. Math. Phys. (2006), pp. 984-998. MR2279252 (2008e:76004) 
[9] Chen, Y., The weak solutions to the evolution problems of harmonic maps, Math. Z., 201 (1989), pp. 69-74. MR990189 (90i:58030)

[10] Ciarlet, P. G., Basic error estimates for elliptic problems: Finite element methods, Part 1, Handbook of Numerical Analysis, P. G. Ciarlet and J. L. Lions, eds., North-Holland, Amsterdam, 1991. MR 1115237

[11] Coutand, D., Shkoller, S., Well-posedness of the full Ericksen-Leslie model of nematic liquid crystals, C. R. Acad. Sci. Paris, Ser. I, 333 (2001), pp. 919-924. MR1873808 (2002j:82118)

[12] Dauge, M., Neumann and mixed problems on curvilinear polyhedra, Integr. equat. Oper. Th. 15 (1992), pp. 227-261. MR1147281 (93e:35025)

[13] Dauge, M., Stationary Stokes and Navier-Stokes systems on two or three-dimensional domains with corners, SIAM J. Math. Anal. 20, 1 (1989), pp. 74-97. MR977489 (90b:35191)

[14] Girault, V. and Lions, J.-L., Two-grid finite-element schemes for the transient Navier-Stokes problem, M2AN 35 (2001) pp. 945-980. MR1866277 (2003a:76078)

[15] Girault, V. and Raviart, P. A., Finite Element Methods for Navier-Stokes Equations. Theory and Algorithms, SCM 5, Springer-Verlag, Berlin, 1986. MR851383 (88b:65129)

[16] Grisvard, P., Elliptic Problems in Nonsmooth Domains, Pitman Monographs and Studies in Mathematics 24, Pitman, Boston, MA, 1985. MR775683 (86m:35044)

[17] Guillén-González, F. and Rojas-Medar, M. A., Global solution of nematic crystals models, C. R. Acad. Sci. Paris, Ser. I, 335 (2002), pp. 1085-1090. MR1955593 (2004a:76007)

[18] Guillén-González, F., Rodríguez-Bellido, M. A., and Rojas-Medar, M. A., Sufficient conditions for regularity and uniqueness of a $3 D$ nematic liquid crystal model. Math. Nach. 282 6, (2009), pp. 846-867. MR2530884

[19] Guillén-González, F., Gutiérrez-Santacreu, J. V., A linear mixed finite element scheme for a nematic Eriksen-Leslie liquid crystal model. Submitted.

[20] Guillén-González, F., Tierra-Chica, G., On pressure error estimates for linear mixed finite element schemes applied to some incompressible fluid models. In preparation.

[21] Jerrison, D. and Kenig, C., The inhomogeneous Dirichlet problem in Lipschitz domains, J. Funct. Anal., 130 1, (1995), pp. 161-219. MR1331981 (96b:35042)

[22] Kellog, R. B. and Osborn, J. E., A regularity for the Stokes problem in a convex polygon, J. Funct. Anal. 21 (1976), pp. 397-431. MR0404849(53:8649)

[23] Lin, F.H., Nonlinear theory of defects in nematic liquid crystals: Phase transition and flow phenomena, Comm. Pure Appl. Math., 42 (1989), pp. 789-814. MR.1003435 (90g:82076)

[24] Lin, F.H. and Liu, C., Non-parabolic dissipative systems modelling the flow of liquid crystals, Comm. Pure Appl. Math., 48 (1995), pp. 501-537. MR.1329830(96a:35154)

[25] Lin, F.H. and Liu, C., Existence of solutions for the Ericksen-Leslie system, Arch. Rat. Mech. Anal., 154 (2000), pp. 135-156. MR.1784963 (2003a:76014)

[26] Lin, P. and Liu, C., Simulations of singularity dynamics in liquid crystal flows: $A C^{0}$ finite element approach. Journal of Computational Physics, 215 (2006), pp. 348-362. MR2215659 (2006m:76009)

[27] Lions, J. L., Quelques Méthodes de Résolution des Problèmes aux Limites Non Linéaires, Dunod, Paris, 1969. MR0259693 (41:4326)

[28] Lions, J. L. and Magenes, E., Problèmes aux Limites non Homogènes et Applications, I, Dunod, Paris, 1968.

[29] Liu, C. and Walkington, N.J., Mixed methods for the approximation of liquid crystal flows, M2AN 36 2, (2002), pp. 205-222. MR1906815 (2003c:76011)

[30] Liu, C. and Walkington, N.J., Approximation of liquid crystal flows, SIAM J. Numer. Anal. 37 3, (2000), pp. 725-741. MR.1740379 (2000k:65174)

[31] Nečas, J., Les méthodes directes en théorie des équations elliptiques, Masson, Paris, 1967.

[32] Prohl, A., Computational Micro-magnetism, Advances in Numerical Mathematics, Teubner 2001. MR1885923 (2004e:82067)

[33] Rannacher, R. and Scott, L. R., Some optimal error estimates for linear finite element approximations, Math. Comp. 38 (1982), pp. 437-445. MR645661(83e:65180)

[34] Scott, L. R. and Zhang, S., Finite element interpolation of non-smooth functions satisfying boundary conditions, Math. Comp. 54 (1990), pp. 483-493. MR.1011446 (90j:65021)

[35] Shkoller, S. Well-posedness and global attractors for liquid crystals on Riemannian manifolds, Comm. Partial Differ. Eq. 27, 5 \& 6 (2001), pp. 1103-1137. MR.1916558 (2003f:37152) 
[36] Simon, J., Compact sets in $L^{p}(0, T ; B)$, Ann. Mat. Pura Appl. 146 (1987), pp. 65-97. MR.916688 (89c:46055)

[37] Temam, R., Navier-Stokes Equations, Theory and Numerical Analysis, North-Holland, Amsterdam, 1979. MR603444 (82b:35133)

Laboratoire Jacques-Louis Lions, Université Pierre et Marie Curie, 75252 Paris CEDEX 05, France

Departamento de Ecuaciones Diferenciales y Análisis Numérico, Universidad de Sevilla, Aptdo. 1160, 41080 Sevilla, Spain 